\title{
Polaron-Mediated Luminescence in Lithium Niobate and Lithium Tantalate and Its Domain Contrast
}

\author{
Philipp Reichenbach 1,* (D), Thomas Kämpfe ${ }^{1}$, Alexander Haußmann ${ }^{1}$, Andreas Thiessen ${ }^{1}$, \\ Theo Woike ${ }^{2}$, Robin Steudtner ${ }^{3}$, Laura Kocsor 4 (iD), Zsuzsanna Szaller ${ }^{4}$ (iD, László Kovács 4 (iD \\ and Lukas M. Eng 1 (ID) \\ 1 Institut für Angewandte Physik, Technische Universität Dresden, Nöthnitzer Str. 61, \\ 01187 Dresden, Germany; thomas.kaempfe@ipms.fraunhofer.de (T.K.); \\ alexander.haussmann@tu-dresden.de (A.H.); thiessen.andreas@web.de (A.T.); \\ lukas.eng@tu-dresden.de (L.M.E.) \\ 2 Institut für Festkörper und Materialphysik, Technische Universität Dresden, Haeckelstraße 3, \\ 01069 Dresden, Germany; theo.woike@tu-dresden.de \\ 3 Institut für Ressourcenökologie, Helmholtz-Zentrum Dresden-Rossendorf, Bautzner Landstraße 400, \\ 01328 Dresden, Germany; r.steudtner@hzdr.de \\ 4 Wigner Research Centre for Physics, Hungarian Academy of Sciences, Konkoly-Thege M. ut 29-33, \\ 1121 Budapest, Hungary; kocsor.laura@wigner.mta.hu (L.K.); szaller.zsuzsanna@wigner.mta.hu (Z.S.); \\ kovacs.laszlo@wigner.mta.hu (L.K.) \\ * Correspondence: philipp.reichenbach@web.de
}

Received: 6 April 2018; Accepted: 29 April 2018; Published: 15 May 2018

\begin{abstract}
In this review article, we discuss photoluminescence phenomena mediated by polarons in lithium niobate (LNO). At first we present the fundamentals on polaron states in LNO and their energy levels, i.e., on free and bound electron polarons, on hole polarons as well as on bipolarons. We discuss the absorption measurements on reduced as well as on doped LNO that made the characterization of the formed polaron states possible by their absorption bands. Next, we proceed by reporting on the two polaron-mediated photoluminescence bands that have been observed in LNO: (1) A near-infrared luminescence band in the range of $1.5 \mathrm{eV}$ shows a mono-exponential decay and a strong dependence on iron doping. This luminescence is emitted by bound polarons returning from an excited state to the ground state. (2) A luminescence band at visible wavelengths with a maximum at $2.6 \mathrm{eV}$ shows a stretched-exponential decay and is strongly enhanced by optical damage resistant doping around the doping threshold. This luminescence stems from the recombination of free electron and hole polarons. The next major topic of this review are domain contrasts of the visible photoluminescence that have been observed after electrical poling of the substrate, as singly inverted domains show a slightly reduced and faster decaying luminescence. Subsequent annealing results in an exponential decrease of that domain contrast. We show that this contrast decay is strongly related to the mobility of lithium ions, thus confirming the role of polar defect complexes, including lithium vacancies, for these domain contrasts. Finally we discuss the extension of our investigations to lithium tantalate (LTO) samples. While the results on the domain contrast and its decay are similar to LNO, there are remarkable differences in their luminescence spectra.
\end{abstract}

Keywords: lithium niobate; lithium tantalate; ferroelectric; polaron; multiphoton luminescence; defect; defect complex; doping threshold; domain contrast; thermal relaxation

\section{Introduction and Overview}

Polarons play an important role in the optical and electrical properties of lithium niobate (LNO), lithium tantalate (LTO) and other ferroelectrics. They are quasiparticle states formed by excited charge 
carriers and the deformation of the surrounding crystal lattice due to their Coulomb forces [1]. Polarons are formed through the relaxation of excited electrons from the conduction band or holes from the valence band into the respective quasiparticle states, i.e., electron or hole polarons. Despite LNO to be exceptionally transparent over a wide wavelength range, reduction, doping and optical excitation can increase the absorption. This emergent absorption in the visible range is commonly associated with the formation of polarons. The governing physics of polarons in oxides and especially LNO has already been the subject of several review articles, yet solely discussing the induction of absorption [2-7]. In this review article, however, we focus on the photoluminescence effects being observed in LNO and LTO that are mediated by these polarons. The corresponding photoluminescence spectra show a broad distribution, one in the visible, the other in the near-infrared wavelength range.

After giving a shorter overview in this introductory part, the investigation of polarons by absorption measurements and the polaronic luminescence phenomena in LNO will be discussed in the second part of this review, preceded by a brief report on defects and bandgap in LNO. The third part deals with domain contrasts of the visible luminescence band in LNO. By these domain contrasts, the luminescence can be used to map switched domains and modifications of the crystal structure therein. The fourth part reviews the results of similar measurements on lithium tantalate (LTO), and compares them with the results from LNO.

In LNO several kinds of polaron states are known to exist [1]. Firstly, there are electron polarons on $\mathrm{Nb}$-sites. The energy of an electron polaron is minimized when it is localized at the ion of highest positive charge, $\mathrm{Nb}_{\mathrm{Nb}}^{5+}$, converting it into the $\mathrm{Nb}_{\mathrm{Nb}}^{4+}$ state. The free electron polaron can move through the crystal by hopping between neighbored niobium ions [1]. Therefore it is called free polaron and is abbreviated by $\mathrm{Nb}_{\mathrm{Nb}}^{4+}$. Secondly, there are electron polarons localized at the niobium anti-sites $\mathrm{Nb}_{\mathrm{Li}}$ that occupy lithium vacancies. These bound polarons $\mathrm{Nb}_{\mathrm{Li}}^{4+}$ are trapped at these defects at an energy below the free polaron energy. Furthermore, a pair of a bound and a free electron polaron can form a bipolaron $[3,5]$.

Photo-excited holes minimize their energy by relaxing into hole polaron states localized at the negatively charged oxygen sites, resulting singly-negatively charged $\mathrm{O}^{-}$ions instead of the doubly-negatively charged $\mathrm{O}^{2-}$ ions [2]. Hole polarons can probably also either be free or bound at lithium vacancies $\mathrm{V}_{\mathrm{Li}}$. An absorption spectrum with a maximum around $2.5 \mathrm{eV}$ and a width of about $1 \mathrm{eV}$ could already be assigned to bound hole polarons [2,3,5,8-12]. About the energy level and properties of free hole polarons in LNO, however, almost nothing is known.

Figure 1 presents an energy level diagram of all these known polaron states and their transitions as well as of the levels of ionic iron defects:

1. free polarons (electrons localized at $\mathrm{Nb}$ ions on $\mathrm{Nb}$ sites: $\mathrm{Nb}_{\mathrm{Nb}}^{4+}$ );

2. bound polarons (electrons localized at $\mathrm{Nb}$ ions on $\mathrm{Li}$ sites: $\mathrm{Nb}_{\mathrm{Li}}^{4+}$ );

3. bipolarons as aggregates of each a bound and a free polaron: $\mathrm{Nb}_{\mathrm{Nb}}^{4+}+\mathrm{Nb}_{\mathrm{Li}}^{4+}$;

4. free hole polarons localized at oxygen ions $\mathrm{O}^{-}$;

5. bound hole polarons localized at oxygen ions $\mathrm{O}^{-}$adjacent to defects, probably lithium vacancies $\mathrm{V}_{\mathrm{Li}}$;

6. $\mathrm{Fe}^{2+}$ and $\mathrm{Fe}^{3+}$ levels: $\mathrm{Fe}^{2+}$ can change into $\mathrm{Fe}^{3+}$ by $\mathrm{e}^{-}$donation that forms an electron polaron state in the neighborhood.

The iron defect levels are also listed due to their importance for the experiments to be discussed below.

The excitation of a bound polaron towards the edge of the conduction band and its subsequent relaxation back to the bound polaron ground state results in a near-infrared photoluminescence with photon energies in the range of $1.5 \mathrm{eV}$. This luminescence was observed especially in iron-doped LNO with intensities depending on the iron doping level $[13,14]$. The recombination of free electron polarons with free hole polarons, on the other hand, yields a luminescence in the visible range with a spectral maximum at $2.6 \mathrm{eV}$ and showing a stretched-exponential decay [15-18]. It increases strongly upon cooling below $200 \mathrm{~K}$ as well as upon optical resistant dopant concentration above the doping 
threshold. The latter observations have been made with $\mathrm{Mg}$-doped samples. This increase with doping concentration expresses the existence of concurring non-radiative recombination processes that depend on the presence of intrinsic defects like $\mathrm{Nb}_{\mathrm{Li}}$ anti-sites. The disappearance of these defects at higher dopings quenches this nonradiative recombination, yielding a stronger luminescence. On doping levels around the doping threshold, however, the strongest temperature dependence of the visible luminescence emerges, as its intensity grows by more than to two orders of magnitude when cooling the samples down to $100 \mathrm{~K}$ and below. This may indicate other nonradiative decay channels occurring in doped LNO that are very sensitive to temperature.

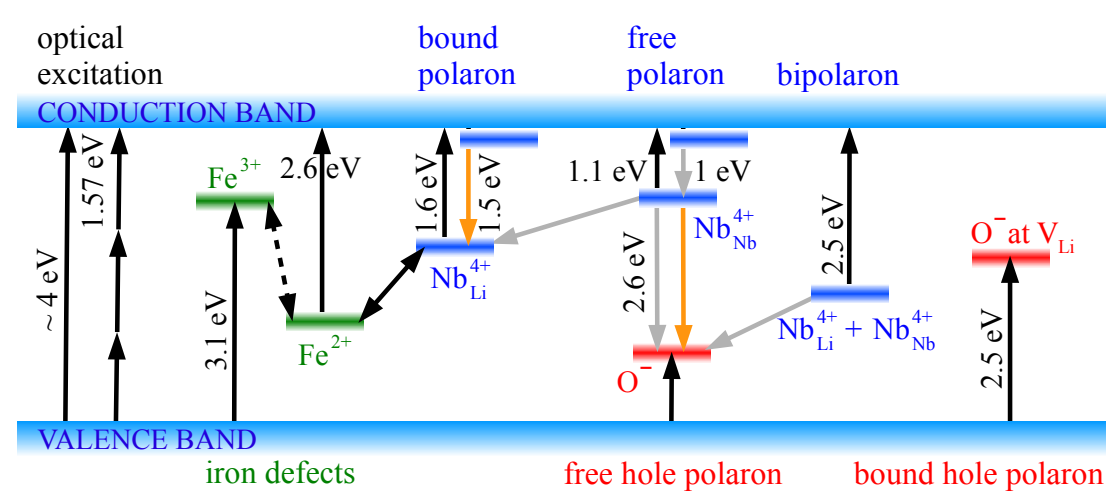

Figure 1. Sketch of the bandgap, energy levels of electron polarons (blue), hole polarons (red) and of ionic iron defects (green). The transitions are depicted by colored arrows: black arrows for all optical absorptions (UV inter-band excitation, three-photon inter-band excitation ...), orange arrows for NIR photoluminescence of the bound polaron $\mathrm{Nb}_{\mathrm{Li}}^{4+}$ and for luminescent recombination of free electron polarons $\mathrm{Nb}_{\mathrm{Nb}}^{4+}$ with free hole polarons $\mathrm{O}^{-}$, and gray arrows for non-radiative relaxations. The relaxations from the conduction band into the bound and free polarons are mediated by shallowly trapped states just below the bandgap [1]. The arrows from and to the $\mathrm{Fe}^{2+}$ state denote the transition of $\mathrm{Fe}^{2+}$ into $\mathrm{Fe}^{3+}$ (dashed line) by donating an electron occupying a bound polaron state in the neighborhood. In the reverse process the electron is transferred from the bound polaron state back to the $\mathrm{Fe}^{3+}$ site, converting the ion back into the $\mathrm{Fe}^{2+}$ state.

The second part of this paper reviews the experimental proofs of the polaron states by absorption measurements, followed by a thorough discussion of the described polaronic luminescence phenomena.

The contrast of the visible photoluminescence between the virgin substrate and electrically inverted domains [17-19] is reviewed in the third part. Similar to Raman lines [20-23] or the coercive voltages of ferroelectric switching [24-28], the radiative polaron recombination efficiency is affected by structural differences between virgin and electrically inverted domains, hence giving rise to the photoluminescence domain contrast. These structural differences are caused by polar defect complexes that do not change their orientation during electric inversion. Thus, the visible luminescence band can be used as a probe for such kind of crystal modifications upon electrical inversion. Up to now, however, no measurements have been reported on a similar domain contrast of the near-infrared luminescence.

The domain contrast decays exponentially under annealing at temperatures above $100{ }^{\circ} \mathrm{C}$ with an activation energy of about $1 \mathrm{eV}$. This activation energy indicates that the thermal contrast decay is related to the mobility of lithium ions [18]. As there are polar defect complexes containing lithium vacancies, the mobility of lithium ions explains well the contrast decay by a thermally induced reorientation of these defect complexes. The mobility of the lithium ions enables a rearrangement of the lithium vacancies. The result is that the orientation of these dipoles follows now the inverted polarization and the dipoles do not longer contribute to the luminescence domain contrast. Hence, radiative recombination can be used to investigate structural differences of virgin versus inverted LNO and even the thermal relaxation of these modifications.

In the fourth part, we append our investigations on the visible-range photoluminescence in Mg-doped congruent LTO (lithium tantalate), its spectral properties and domain contrast. This material 
has been investigated due to its high structural similarity with LNO. We observed the visible luminescence peak in the blue wavelength range, and even stronger domain contrasts than in LNO with a similar behavior of thermal contrast decay. A phenomenon with no counterpart in LNO is the vanishing of the blue peak and the revealing of another peak in the green wavelength range at smaller excitation powers as well as in undoped LTO. As there is rather little knowledge of polaronic levels and activation energies in LTO, these data and their comparison to LNO give interesting first insights into that field that could be still extended and clarified by deeper research.

In the last part we summarize our review and present an outlook on still unsolved questions.

\section{Luminescence and Absorption by Polarons in LNO}

\subsection{Defects, Bandgap, and Relaxation of Excited Charge Carriers in LNO}

In this subsection we discuss some aspects of the material physics of LNO with big importance for the physics of polarons and polaronic luminescence. The first point are the defects and defect complexes. In LNO defects appear not only by doping but also by the slight deviations of the $\mathrm{Li}: \mathrm{Nb}$ ratio from 1:1 that occur in almost all variants of LNO. The second point are the bandgap of LNO and the relaxation time of charge carriers that have been excited across this bandgap.

The defects occurring in undoped and Mg-doped LNO have a strong impact on the measured luminescence intensity, especially on the visible luminescence. At first we discuss the defects in undoped LNO. The widely used congruently-grown lithium niobate (CLN) has a $\mathrm{Li} /(\mathrm{Li}+\mathrm{Nb})$ ratio of $48.45 \%$ [1]. LNO prepared to have a $\mathrm{Li} /(\mathrm{Li}+\mathrm{Nb})$ ratio closer to $50 \%$ is called stoichiometric lithium niobate (SLN). In general SLN is also only near-stoichiometric, the $\mathrm{Li} /(\mathrm{Li}+\mathrm{Nb})$ ratio is not exactly at $50 \%$. In the 70's, the stoichiometry of such LNO samples has been still nearer to CLN than to perfect stoichiometric LNO, while in later times stoichiometry approached the perfect balance much better.

In all these samples without perfect stoichiometry $(\mathrm{Li} /(\mathrm{Li}+\mathrm{Nb})<50 \%)$ the lithium deficiency results in the presence of lithium vacancies $\mathrm{V}_{\mathrm{Li}}$ and niobium ions placed on lithium sites (so-called lithium anti-sites) $\mathrm{Nb}_{\mathrm{Li}}$ [1]. The lithium deficiency is then related to the density of these so-called intrinsic defects. Usually, these defects are aggregated in defect clusters where the effective charges of the constituting defects compensate each other. Several models of defect complexes formed by lithium or niobium vacancies and $\mathrm{Nb}$ anti-sites have been proposed $[1,22,29,30]$. The most important variant is the defect complex formed by a $\mathrm{Nb}_{\mathrm{Li}}$ anti-site, surrounded by four lithium vacancies $\mathrm{V}_{\mathrm{Li}}$. In the ionic crystal the charges of the constituting ions $\left(1 \times \mathrm{Li}^{+}, 1 \times \mathrm{Nb}^{5+}\right.$ and $\left.3 \times \mathrm{O}^{2-}\right)$ compensate in each unit cell. Vacancies or defect ions cause deviations from these charges at the places of the crystal lattice, which we call here effective charges. $\mathrm{A} \mathrm{Nb}^{5+}$ ion on a $\mathrm{Li}$ site (hosting normally $\mathrm{a} \mathrm{Li}^{+}$ion) yields a local charge excess of $4+: \mathrm{Nb}_{\mathrm{Li}}^{(4+)}$, while a lithium vacancy (one positive charge lacking) has a negative effective charge: $\mathrm{V}_{\mathrm{Li}}^{(-)}$. Therefore the defect cluster $\mathrm{Nb}_{\mathrm{Li}}^{(4+)}+4 \mathrm{~V}_{\mathrm{Li}}^{(-)}$is charge-compensated. Another possible charge-compensated defect cluster model involves also niobium vacancies (with an effective charge of $5-): 5 \mathrm{Nb}_{\mathrm{Li}}^{(4+)}+4 \mathrm{~V}_{\mathrm{Nb}}^{(5-)}[1,2,24,30]$. NMR measurements and subsequent simulations showed that the $\mathrm{Nb}_{\mathrm{Li}}^{(4+)}+4 \mathrm{~V}_{\mathrm{Li}}^{(-)}$defect cluster is most probable in LNO [28,31,32], although DFT energy calculations indicated that the $5 \mathrm{Nb}_{\mathrm{Li}}^{(4+)}+4 \mathrm{~V}_{\mathrm{Nb}}^{(5-)}$ complex is energetically similarly preferred, too [30]. Probably both kinds of defect complexes occur in LNO with lithium deficiency. The Li:Nb ratio is determined by the density of these defect clusters.

In addition to these vacancies and anti-sites, there are also niobium interstitials in LNO. In the ideal lithium niobate structure, $1 / 3$ of the oxygen octahedra are empty [1]. In real LNO, however, niobium interstitials $\mathrm{Nb}_{\mathrm{V}}^{5+}$ occur that occupy these ideally empty octahedra [33,34]. Like niobium anti-sites, these niobium interstitials are also accompanied by lithium vacancies and form defect clusters [34].

The presence of these $\mathrm{Nb}_{\mathrm{Li}}$ anti-sites can be strongly reduced by so called damage-resistant doping [1]. Doping with elements like $\mathrm{Mg}, \mathrm{Zn}, \mathrm{Sc}$ - that leave the material transparent-leads to a replacement of $\mathrm{Nb}_{\mathrm{Li}}$ anti-sites by dopant ions placed on lithium sites. An investigation with varying 
Mg doping showed that the Mg ions do not immediately occupy lithium sites when increasing the $\mathrm{Mg}$ concentration from zero. At lowest dopings Mg ions occupy at first oxygen octahedra that are regularly empty; with increasing doping they are also found on lithium sites [35]. Around a certain threshold concentration of the dopant (doping threshold), which depends on the valence of the dopant ions and the stoichiometry of the doped $\mathrm{LNO}$, the $\mathrm{Nb}_{\mathrm{Li}}$ anti-sites have been completely replaced and the dopant ions start to replace also niobium ions $\mathrm{Nb}_{\mathrm{Nb}}$ on niobium sites. It is probable that the niobium intersticials $\mathrm{Nb}_{\mathrm{V}}$ are replaced by $\mathrm{Mg}$ ions as well. As the removal of $\mathrm{Nb}_{\mathrm{Li}}$ anti-sites changes the optical absorption properties, especially by blueshifting the UV absorption band, this doping results in a higher resistance against high laser light densities (optical damage resistance).

In the most investigations presented in this review the used LNO samples are doped with magnesium, a widely used standard dopant for LNO. In CLN, Mg has a doping threshold in the range of 4.5 to $5 \mathrm{~mol} \%$, and the $\mathrm{Nb}_{\mathrm{Li}}$ anti-sites vanish completely between 3 and $5 \mathrm{~mol} \%$ [1]. Due to the residual Li deficiency, also near-stoichiometric SLN has still a finite doping threshold of $1 \mathrm{~mol} \%$ for $\mathrm{Mg}$, as observed by Furukawa et al. [36]. In the group of L. Kovács, however, SLN with an even better stoichiometry was demonstrated showing a doping threshold of only $0.2 \mathrm{~mol} \%$ [37].

Below the doping threshold, the $\mathrm{Mg}_{\mathrm{Li}}^{(+)}$defects with single effective charges $\left(\mathrm{Mg}^{2+}\right.$ on a $\mathrm{Li}^{+}$site $)$are compensated by Li vacancies, resulting in $\mathrm{Mg}_{\mathrm{Li}}^{(+)}+\mathrm{V}_{\mathrm{Li}}^{(-)}$clusters. Above the threshold, the additionally occurring effectively negatively charged $\mathrm{Mg}_{\mathrm{Nb}}^{(3-)}$ can be compensated in an energetically favorable manner by already existing $\mathrm{Mg}_{\mathrm{Li}}^{(+)}$in $\mathrm{Mg}_{\mathrm{Nb}}^{(3-)}+3 \mathrm{Mg}_{\mathrm{Li}}^{(+)}$defect clusters [38].

After reporting the defects occurring in LNO we take a view on the LNO bandgap, which is also of great importance for the discussions of the polarons, their excitations and transitions. The different methods for determining the bandgap, however, yield a broader range of results ranging from about 4 to $5.4 \mathrm{eV}$. With an optical bandgap of $4 \mathrm{eV}$ or larger, LNO is exceptionally transparent.

An important method for determining the bandgap was the measurement and evaluation of spectral absorption curves. This method resulted in bandgap values in a range of about 4 to $4.3 \mathrm{eV}$ [39-42]. The minor absorption in the tail below $4 \mathrm{eV}$ has been assigned to indirect band transitions [40].

The bandgap, as determined by this method, decreases weakly upon elevated temperatures by about $7 \times 10^{-4} \mathrm{eV} / \mathrm{K}$ [39]. The bandgap of SLN showed to be about $0.1 \mathrm{eV}$ larger than that of CLN, as measured by Redfield et al. [39]. As mentioned above, at the time of these investigations perfect stoichiometry was approached worse than in later times. In more recent measurements the bandgap of SLN samples has been determined to be by $0.2-0.3 \mathrm{eV}$ larger than in CLN [43].

Reflection measurements, on the other hand, yielded strong maxima of reflectivity at energies around $5 \mathrm{eV}$ [44-46], indicating the bandgap to be at $5 \mathrm{eV}$.

Theoretical calculations based on DFT and self-consistent self-energy calculations yielded values that rather fit to the results from the reflectivity than to the absorption measurements. Thierfelder et al. calculated a value of $4.7 \mathrm{eV}$ [47], while a more recent and developed calculation resulted even in $5.4 \mathrm{eV}$ [48]. These calculation models were also able to reproduce well the dielectric function of LNO [49].

These straying and seemingly contradictory results show the difficulties to determine the true (direct) bandgap of LNO. One may doubt the interpretation of the absorption measurements, because interactions of positive and negative charge carriers may yield absorption processes below the (real) bandgap by the excitation of certain bound states. On the other hand the calculations may be doubted because they have not been performed over more than a few unit cells, thus not considering the disorder of the real crystal. These open questions show that the exact value of the (direct) bandgap of LNO is still controversial. In the sketch in Figure 1 as well as in some discussions in this review we will refer to the widely used bandgap value of $4 \mathrm{eV}$. However, we put this value and all statements based on it under the reservation that the question of the exact bandgap value is not yet finally resolved.

After photoexcitation, electrons and holes relax into polaron states. For the discussion of polaron luminescence it is very important to consider the time scale of this relaxation as well. Pump-probe 
spectroscopy measurements have shown that photo-excited charge carriers relax into their respective polaron states within hundreds of femtoseconds [50-52]. Recombination of polarons, on the other hand, occurs at time scales of $10^{-7} \mathrm{~s}$ and longer $[15,19]$. Hence, polaron recombination and polaron formation occur at very different time scales. Thus, for the dynamics of polaronic recombination this short relaxation time can be neglected, as well as transport effects by the motion of the photo-excited charge carriers in the conduction band.

To resume the discussion of the bandgap issue, the charge carriers were excited by $4.8 \mathrm{eV}$ into the conduction band via three-photon excitation $[50,51]$, showing that the bandgap lies probably below $4.8 \mathrm{eV}$. In our luminescence experiments, the charge carriers were excited by about $4.7 \mathrm{eV}$-by UV as well as by multiphoton excitation. In that case the cited results on charge carrier relaxation can be well premised for our luminescence measurements.

\subsection{Absorption in LNO by Polarons}

The first way to identify and characterize polaron states and their energies were measurements of their absorption bands after filling these polaron states with charge carriers by reduction. These absorption measurements allowed investigations of the influence of physical parameters on the polarons, thus clarifying their energy levels and physical nature. Doping these samples with $\mathrm{Mg}$ or Fe allowed further insights into the physics of these polaron states.

First light-induced absorption spectroscopy experiments have been carried out by Koppitz et al. [16,53] at congruent lithium niobate (CLN) that had been reduced for $1 \mathrm{~h}$ at $800{ }^{\circ} \mathrm{C}$ in vacuum. These measurements have also been extended to Mg-doped CLN. The absorption spectra of this material revealed several broad absorption bands with characteristic peak energies that could be attributed to the optical bands of electron polarons:

1. Peak around $1.1 \mathrm{eV}$ (about $1130 \mathrm{~nm}$ ), transition from free polaron to conduction band;

2. Peak around $1.6 \mathrm{eV}$ (about $770 \mathrm{~nm}$ ), transition from bound polaron to conduction band;

3. Peak around $2.5 \mathrm{eV}$ (about $500 \mathrm{~nm}$ ), transition from bipolaron to conduction band;

4. Peak around $0.6 \mathrm{eV}$, transition from polarons at $\mathrm{Mg}_{\mathrm{Nb}}$ defects to conduction band, occurring only in CLN with more than $5 \mathrm{~mol} \% \mathrm{Mg}$ doping, not depicted in Figure 1.

The absorption curves (1) to (3) are drawn in Figure 2. The assignments of these spectral features to the according polaron types were supported by the dependence of these absorption peaks on temperature and doping with $\mathrm{Mg}$. With $\mathrm{Mg}$ doping replacing the $\mathrm{Nb}_{\mathrm{Li}}$ defects by $\mathrm{Mg}_{\mathrm{Li}}$ defects [1], both the bound polaron and the bipolaron peaks were reduced [16], indicating a strong relation of these peaks to charge carriers bound to $\mathrm{Nb}_{\mathrm{Li}}$ defects. After excluding other possibilities, the thermal decay of the $2.5 \mathrm{eV}$ peak (3) with an activation energy of $0.27 \mathrm{eV}$ could be explained by the presence of aggregates of free and bound polarons (bipolarons) that decay thermally into single polarons [53]. The $1.6 \mathrm{eV}$ peak (2), on the other hand, has been assigned to single bound polarons [53]. The dissociation of bipolarons has also been observed under illumination with green or blue light, leading to enhanced transparency in the blue wavelength range and increased absorption in the red and near-infrared wavelength range by the shift of the absorption spectrum from the bipolaron peak (3) to the bound polaron peak (2) [54-56]. The attribution of the $1.6 \mathrm{eV}$ absorption band to the $\mathrm{Nb}_{\mathrm{Li}}^{4+}$ state has been finally confirmed by measurements of magnetic circular dichroism of this absorption center [57].

The $1.1 \mathrm{eV}$ peak (1) on the other hand remains stable for high Mg doping and under annealing, thus being independent from $\mathrm{Nb}_{\mathrm{Li}}$ and other defects. Therefore that peak has been assigned to the free polarons on $\mathrm{Nb}_{\mathrm{Nb}}$ places. The 0.6 peak (4), not shown in Figure 2, appears for $\mathrm{Mg}$ dopings above the doping threshold of $5 \mathrm{~mol} \%$, where $\mathrm{Mg}$ starts also to replace $\mathrm{Nb}$ ions from $\mathrm{Nb}$ places [1]. This peak probably stems from polarons slightly trapped at the occurring $\mathrm{Mg}_{\mathrm{Nb}}$ defects [16]. The formation of this peak showed an activation energy of $40 \mathrm{meV}$ [16]. 


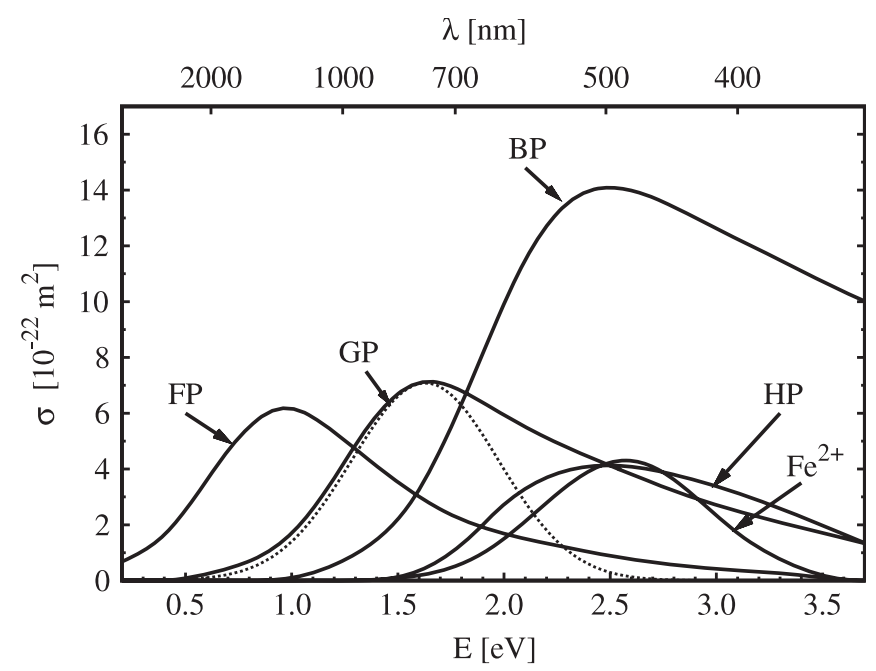

Figure 2. Spectral absorption cross sections calculated by Merschjann et al. [5] for different kinds of polarons and $\mathrm{Fe}^{2+}$ centers in CLN at room temperature. The calculated curves are based on experimental data from Refs. [3,53,58,59]. The absorption spectra belong to the following defect centers: Free electron polarons (FP), bound electron polarons (denoted as GP), bipolarons (BP), bound hole polarons (HP) and $\mathrm{Fe}^{2+}$ ions. The dotted curve shows a theoretical band shape of bound electron polarons according to Refs. [60,61]. This figure is reproduced with permission from C. Merschjann et al., J. Phys. Condens. Mater. 2009, 21, 015906 [5], copyright (C) 2009 by IOP Publishing Ltd.

For reduced or n-doped (e.g., Fe or Mg) LNO electric transport can be assumed to be dominated by hopping of free polarons [62]. Even for nominally undoped LNO the same can be assumed due to small amounts of Fe ions generally occurring there. In these cases investigations of the electrical transport in LNO and comparison with the theoretical polaron model have provided further support for the energy level of free electron polarons. According to the polaron model $[4,58,63]$ the electron and the surrounding lattice distortion have a total energy $E_{\mathrm{P}}$, while the electron itself has an energy $E_{\mathrm{opt}}=2 E_{\mathrm{P}}$, which is relevant for optical absorption. Then the activation energy $E_{\mathrm{h}}$ for hopping to neighboring $\mathrm{Nb}_{\mathrm{Nb}}$ sites is $E_{\mathrm{h}}=E_{\mathrm{P}} / 2=E_{\mathrm{opt}} / 4$. Reported activation energies for electrical transport in reduced or doped LNO are between 0.1 and $0.24 \mathrm{eV}[58,64,65]$, which is in a fairly good agreement with the absorption band around $1 \mathrm{eV}$ for free polarons, therefore resulting in a theoretical hopping energy of about $0.25 \mathrm{eV}$ [4]. However, the relation $E_{\mathrm{h}}=E_{\mathrm{P}} / 2$ is only an approximation and, moreover, $E_{\mathrm{h}}$ is often smaller than $E_{\mathrm{P}} / 2$ [66], which meets the range of measured values even better. Note that the polaronic model itself is not valid at arbitrarily low temperatures. There are models that generally predict a metal-insulator transition for polarons at low temperatures, resulting in an itinerant polaron band, while inelastic processes at higher temperatures yield the known hopping behavior [66-69].

While these findings cover all electron polaron states, the knowledge of hole polarons and their transitions is much more fragmentary, as only the absorption band of the bound hole polaron is known and almost nothing about the free hole polaron. Under two-photon-photoexcitation of undoped CLN with $532 \mathrm{~nm}$ picosecond pulses at a temperature of $20 \mathrm{~K}$, an absorption band around $2.5 \mathrm{eV}(500 \mathrm{~nm}$, see Figure 2) has been measured by Schirmer et al. [8,9]. Comparison with broad ESR spectra at $\mathrm{O}^{2-}$ ions (and the knowledge of similarly broad hole absorption spectra in other materials) resulted in the assignment of this peak to bound hole polarons that are probably bound to lithium vacancies $[2,9,11,70]$. Compared to electron polarons the absorption energy of $2.5 \mathrm{eV}$ is rather high, however, this seems to be typical for $\mathrm{O}^{-}$hole polarons in oxides [70]. At room temperature or under additional HeNe laser light illumination this peak is still visible, but narrower and considerably weaker [8,9]. This influence of HeNe laser light may indicate a recombination of the bound hole polarons with electron polarons or conversions of the bound hole polarons into free hole polarons. More recent pump-probe investigations on CLN by Herth et al. [12] measured decay times of about milliseconds for that absorption band, 
at room temperature and also under picosecond excitation at $532 \mathrm{~nm}$. As no free polaron band was observed under these excitation conditions, the formation of bipolarons as a cause of this band has been excluded [12,71] and the assignment to generated bound holes has been confirmed [12]. However, almost no data is known about the energy levels and physical properties of free hole polarons.

Ionic iron defects and their energy levels are very important for the infrared polaronic luminescence to be reviewed below. For that reason it is necessary to discuss here some additional findings on absorption measurements on iron doped CLN as well as on transitions between Fe defect ion states and polaron states. Fe defect ions are known to almost exclusively occupy Li sites [59,72,73]. Note that small iron impurities are generally present in every LNO sample. Higher Fe doping, however, will strongly increase all optical effects caused by these ions.

In Fe-doped CLN an absorption band around $2.6 \mathrm{eV}$ has been found (see Figure 2)[59,74] that has been attributed to the $\mathrm{Fe}^{2+} \rightarrow \mathrm{Fe}^{3+}+\mathrm{e}^{-}$excitation process [74]. Investigations of Kurz et al. included the reduction of Fe-doped CLN samples by annealing and monitoring their $\mathrm{Fe}^{3+}$ and $\mathrm{Fe}^{2+}$ concentrations using Mössbauer and EPR measurements. These experiments resulted in a strong linear correlation between $\mathrm{Fe}^{2+}$ concentration and absorption at the $2.6 \mathrm{eV}$ band, thus confirming the attribution of this band to the $\mathrm{Fe}^{2+} \rightarrow \mathrm{Fe}^{3+}+\mathrm{e}^{-}$excitation process [59]. Another charge-transfer $\mathrm{O}^{2-}+\mathrm{Fe}^{3+} \rightarrow \mathrm{O}^{-}+\mathrm{Fe}^{2+}$ has been mentioned by Schirmer et al. and attributed to an absorption band around $3.1 \mathrm{eV}$ [2].

Furthermore, the generation of bound polarons by photo-excitation of electrons from $\mathrm{Fe}^{2+}$ ions has been demonstrated by exciting Fe-doped LNO crystals with nanosecond laser light pulses at $532 \mathrm{~nm}$. This excitation resulted in an increased absorption in the red wavelength range [71,75]. These experiments have been carried out with different concentrations of $\mathrm{Fe}^{2+}$ and $\mathrm{Nb}_{\mathrm{Li}}$, using CLN as well as SLN and further variations of stoichiometry. The observed absorption increased with $\mathrm{Fe}^{2+}$ concentration, but was weaker in samples with lower $\mathrm{Nb}_{\mathrm{Li}}$ concentration. Moreover, a decrease in the concentration of $\mathrm{Fe}^{2+}$ ions and of $\mathrm{Nb}_{\mathrm{Li}}$ increased the transition time of the growing absorption [75,76]. This supports that the pump light excites a charge transfer at $\mathrm{Fe}^{2+}$, creating a polaron, leaving a $\mathrm{Fe}^{3+}$ ion behind and yielding an increase in absorption in the red and near-infrared wavelength range. The rate of this reaction depends then on both the $\mathrm{Fe}^{2+}$ and $\mathrm{Nb}_{\mathrm{Li}}$ anti-site concentrations. The relation of this phenomenon with the absorption peak in the near-infrared and the concentration of $\mathrm{Nb}_{\mathrm{Li}}$ anti-sites indicates that the involved polaron is indeed the bound polaron. This has been confirmed by the time-dependent investigations on the absorption at $2.5 \mathrm{eV}$ by Herth et al. [12]. Pump-probe absorption measurements of $0.1 \mathrm{~mol} \% \mathrm{Fe}: \mathrm{CLN}$ under nanosecond-pulsed illumination at $532 \mathrm{~nm}$ showed an increase of the $\mathrm{Fe}^{2+}$ absorption band within the first $10^{-4} \mathrm{~s}$ after the exciting pulse. This increase showed a correlation with the decay of the bound polaron band at the same time. Thus, the increase of the $\mathrm{Fe}^{2+}$ concentration could be directly correlated with the decrease of bound polaron concentration, as the bound polarons (created through the exciting pulse) recombine again with the $\mathrm{Fe}^{3+}$ ions to $\mathrm{Fe}^{2+}$ ions [12]. A direct transition from $\mathrm{Fe}^{2+}$ into free electron polaron states by optical absorption, however, has not been reported-probably because such a transition is forbidden.

After pumping, the relaxation of the bound polaron absorption in the red wavelength followed a stretched-exponential law. The relaxation of these bound polaron states back to $\mathrm{Fe}^{2+}$ states means a return of the negative charge carriers from the hosting $\mathrm{Nb}_{\mathrm{Li}}$ sites to the $\mathrm{Fe}^{3+}$ ions, converting them back to the $\mathrm{Fe}^{2+}$ state. As the nearest neighbor distances of $\mathrm{Nb}_{\mathrm{Li}}$ and iron sites are randomly distributed, the decay of excited bound polarons follows a superposition of exponential decays with different time constants, resulting in a stretched-exponential behavior with decay times in the microsecond range [77]. Like the transition rate mentioned above, this decay process slowed down at smaller densities of $\mathrm{Nb}_{\mathrm{Li}}$ anti-sites due to the resulting larger average distance to iron defects [75,76]. A deeper analysis of the physics of stretched-exponential luminescence has already been given for the example of the luminescence of Si nanocrystals [78]. As will be discussed below, Herth et al. [71] confirmed this behavior for the bound polarons in CLN. Furthermore, they found also reduced polaron lifetimes after introducing co-dopants such as $\mathrm{Cu}$, providing more decay channels. An Arrhenius-like behavior of 
the polaron decay with an activation energy of $0.38 \mathrm{eV}$ was observed, too [71]. If we would use the polaron hopping model for the escape of the charge carrier from the $\mathrm{Nb}_{\mathrm{Li}}^{4+}$ state back to the iron ion, this activation energy would well agree with the optical absorption energy of $1.6 \mathrm{eV}$.

\subsection{Near-Infrared Photoluminescence Band in LNO}

Up to now we have discussed the different polaron states occurring in LNO and their physical behavior. This is a necessary basis to understand the photoluminescence phenomena that are mediated by these polaron states. In this section, we will discuss the near-infrared luminescence observed around 1.3-1.5 eV. It has been shown that this band stems from the relaxation of excited bound polarons and that its intensity depends also strongly on the presence of ionic iron defects. The latter is the case due to the role of iron ions for the generation of bound polarons by photoexcitation.

First measurements of a luminescence band around $1.5 \mathrm{eV}$ have been carried out in slightly Fe-doped CLN $(0.015 \mathrm{~mol} \% \mathrm{Fe})$ upon UV laser light illumination at $325 \mathrm{~nm}$ [79]. In untreated Fe-doped samples, the luminescence is weak, while it is strongly enhanced after reduction (by annealing for $20 \mathrm{~h}$ at $520{ }^{\circ} \mathrm{C}$ in $\mathrm{LiCO}_{3}$ ). Due to the donated electrons, the charge transfer $\mathrm{Fe}^{2+} \rightarrow \mathrm{Fe}^{3+}+\mathrm{e}^{-}$resulting from the reduction process $[80,81]$ indicates a strong relation between that luminescence and the presence of polarons.

Further investigations $[13,82]$ on this luminescence band of undoped LNO yielded deeper insights into the physical origin of this luminescence-especially confirming its mediation by bound polarons. Exciting undoped LNO with ns pulses at either $355 \mathrm{~nm}$ [13] or $514 \mathrm{~nm}$ [82] resulted in two infrared luminescence peaks at $1.5 \mathrm{eV}(835 \mathrm{~nm})$ and $1.3 \mathrm{eV}(950 \mathrm{~nm})$, as shown in Figure 3a [13,83].
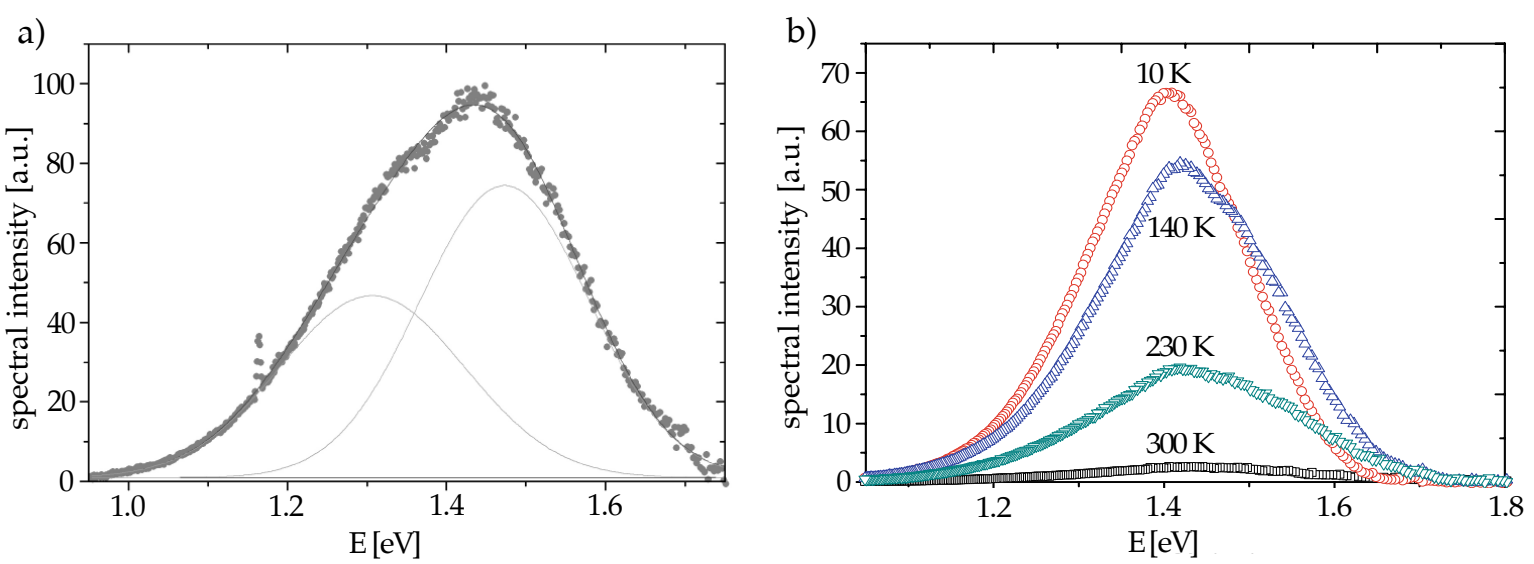

Figure 3. (a) NIR photoluminescence peak from undoped CLN excited by a pulsed $335 \mathrm{~nm}$ beam measured by Harhira et al. The whole peak shows a maximum around $1.4 \mathrm{eV}$ and can be fitted by two Gaussian peaks at 1.3 and $1.5 \mathrm{eV}$ as luminescence centers [13,83]. This figure is reproduced with permission from A. Harhira, Photoluminescence polaron dans le niobate de lithium, Ph.D. Thesis, 2010 [83]. (b) NIR photoluminescence peak for 0.02\% Fe:CLN excited by a CW $633 \mathrm{~nm}$ laser beam for different temperatures [14]. Note the slight spectral shift of the peak from 10 to $300 \mathrm{~K}$. This figure is reproduced with permission from A. Harhira et al., Appl. Phys. B 2008 92, 555 [14], copyright (C) 2008 by Springer.

These two peaks are no fitting artifacts, as the following results show. Kostritskii et al. showed that the intensity of the $1.5 \mathrm{eV}$ peak depends strongly on the intrinsic defect concentration, which has been revealed by comparison of congruent lithium niobate (CLN) and stoichiometric lithium niobate (SLN) [82,84]. In CLN the $1.5 \mathrm{eV}$ and the $1.3 \mathrm{eV}$ peaks amounted to the same order of magnitude; in SLN, however, the $1.5 \mathrm{eV}$ peak was significantly weaker while the $1.3 \mathrm{eV}$ peak did not change drastically. Assuming a connection of the $1.5 \mathrm{eV}$ luminescence peak to the bound polaron state, this behavior fits again well with the reduced presence of lithium vacancies and therefore also of $\mathrm{Nb}_{\mathrm{Li}}$ 
defects that can provide bound polarons in SLN. This connection to the bound polaron state is also confirmed by a strong increase of the $1.5 \mathrm{eV}$ peak in CLN upon prolonged reduction [82,84].

The $1.3 \mathrm{eV}$ peak, however, vanishes under that reduction. This may indicate a relation of that peak to hole polarons, as the reduction floods the substrate with negative charge carriers not only appearing as electron polarons but also filling the remaining holes. Maybe it is even a counterpart of the bound polaron luminescence for free or bound holes-a transition from free hole states or from excited bound hole states back to the bound hole ground state. Unfortunately, no further investigations have been reported for that peak so far. Therefore no data are available for the determination of the deeper physical nature of that peak - probably because of problems to separate both peaks spectrally. However, we must reject the claim of Kostritskii et al. [82,84] to assign the $1.3 \mathrm{eV}$ peak to the recombination of free electron polarons with hole polarons. The recombination of free electron polarons with free hole polarons has already been assigned to the luminescence in the visible wavelength range to be discussed below. Moreover, the time dependence of such polaronic recombinations would result in a stretched-exponential behavior $[19,76]$, which has not been observed in the time-dependent measurements of the NIR luminescence band to be discussed below.

Further measurements of temporal, thermal, and doping behavior confirmed and clarified the role of the bound polaron state in the $1.5 \mathrm{eV}$ luminescence peak. Unfortunately, in all these measurements to be discussed in the following, no differentiation has been made between the 1.5 and $1.3 \mathrm{eV}$ peaks, possibly due to difficulties to separate these two overlapping peaks. Conclusions have therefore been drawn only for the $1.5 \mathrm{eV}$ peak, and not for the $1.3 \mathrm{eV}$ peak.

Measurements of the $1.5 \mathrm{eV}$ photoluminescence in Fe-doped CLN showed a clear increase of intensity with the doping level, while lifetime and temperature dependence were not influenced [14]. This supports the role of Fe not as a luminescence center itself but as a donor providing bound electron polarons as luminescence centers. The luminescence of the $1.5 \mathrm{eV}$ peak has been interpreted as the result of a two-step excitation process: A charge transfer $\mathrm{Fe}^{2+}+\mathrm{Nb}_{\mathrm{Li}}^{5+} \rightarrow \mathrm{Fe}^{3+}+\mathrm{Nb}_{\mathrm{Li}}^{4+}$ is followed by a transition into an excited state $\left(\mathrm{Nb}_{\mathrm{Li}}^{4+}\right)^{*}$ slightly below the conduction band edge (Figure 1 ). The luminescence energy of $1.5 \mathrm{eV}$ agrees well with the bound polaron energy of $1.6 \mathrm{eV}$, when $\left(\mathrm{Nb}_{\mathrm{Li}}^{4+}\right)^{*}$ is identified with a shallow trap around $0.1 \mathrm{eV}$ below the conduction band edge. The mono-exponential behavior of the luminescence decay (with a unique decay time) supports the interpretation that the bound polaron and excited state $\left(\mathrm{Nb}_{\mathrm{Li}}^{4+}\right)^{*}$ are on the same site, since a random spatial separation would result in a stretched exponential decay as a superposition of exponentials with different decay times $[19,76,77]$.

Moreover, strongly Fe-doped CLN ( $1 \mathrm{~mol} \% \mathrm{Fe})$ showed a quadratic increase of the the luminescence intensity with the illumination intensity, according to a two-step excitation [14]. For lower doping concentrations than $0.05 \mathrm{~mol} \% \mathrm{Fe}$, the dependence of the luminescence on the illumination intensity reached a linear regime, because the smaller number of Fe ions was already completely excited at the used illumination intensities (saturation regime). In this regime, the found direct proportionality between the luminescence intensity and doping concentration supports the model of polaron excitation by charge transfer. The observation of luminescence in nominally undoped LNO may be related to the Fe impurities that are generally present in this material.

In addition to these doping dependencies, Harhira et al. found also a strong temperature dependence of the luminescence intensity, which is typical for polaron-mediated luminescence, as shown in Figure 3b. Due to non-radiative transitions the $1.5 \mathrm{eV}$ luminescence peak decreases strongly with temperature and is at room temperature about 2 orders of magnitude weaker than at $10 \mathrm{~K}$. This dependence on temperature yields an activation energy of $0.22 \mathrm{eV}$. However, the radiative decay time of $9 \mu$ s was observed to be independent on temperature [13]. As the extracted activation energy is near the free polaron hopping energy, the authors interpreted the results in the following way: the temperature dependence is caused by a concurring non-radiative decay being mediated by hopping from the excited state $\left(\mathrm{Nb}_{\mathrm{Li}}^{4+}\right)^{*}$ to the free polaron and further down to the bound polaron (Figure 1) [14]. 
Note that no analogous luminescence had been observed for free polarons, which supports that the relaxation via the free polaron state is indeed completely non-radiative.

This interpretation, however, is still contradictory. When a certain number of bound polarons is excited and an additional temperature-dependent nonradiative decay would occur, the total measured luminescence lifetime would also be influenced by that nonradiative channel and could not be temperature-independent. We suggest here an alternative interpretation: Between the excitation of the charge carrier from the $\mathrm{Fe}^{2+}$ ion into the bound polaron state and the excitation of that polaron, the possibility exists that the charge carrier tunnels to a neighbored $\mathrm{Nb}_{\mathrm{Nb}}$ site. The probability not to return but to escape as a free polaron is determined by the hopping probability being governed by the hopping activation energy. As a result, the number of bound polarons being excited to the $\left(\mathrm{Nb}_{\mathrm{Li}}^{4+}\right)^{*}$ state is drained by that escape process, yielding the polaron hopping activation energy for the temperature dependence of the luminescence. The luminescence process itself, however, would not be accompanied by a nonradiative decay modifying the luminescence lifetime.

\subsection{Visible Luminescence Band in LNO}

The second important polaron-mediated photoluminescence band found in LNO lies in the visible wavelength range. The luminescence maximum was observed around $2.6 \mathrm{eV}$ (ca. $470 \mathrm{~nm}$ ), as shown in Figure $4 \mathrm{a}$. The luminescence showed a strong dependence on $\mathrm{Mg}$ doping and has been associated with the recombination of free electron with free hole polarons [15-19]. This interpretation has been supported by a stretched-exponential decay of this luminescence with lifetimes in the range of $\mu \mathrm{s}$ $[19,71]$. Similar to the transfer of bound polarons into iron ions described above, this recombination involves randomly distributed recombination partners being separated by random distances-resulting in a stretched-exponential instead of a mono-exponential decay [19,71,76,78].

As there is little data about the properties of free hole polarons, the measured luminescence energy can, in principle, be used to determine the hole polaron energy level. Assuming a LNO bandgap of $4-4.3 \mathrm{eV}$ following to the absorption measurements [39-41], a free polaron energy of $1.1 \mathrm{eV}$ and the $2.6 \mathrm{eV}$ luminescence energy leave a free hole polaron absorption energy of about $0.3-0.6 \mathrm{eV}$ (Figure 1). The calculated bandgap values of 4.7-5.4 eV [47,48], on the other hand (supported also by the reflection measurements in Refs. [44,45]), result in higher free hole polaron energies of 1.0-1.7 eV, comparable with the free electron polaron and bound hole polaron energies. A recombination of free polarons with bound holes, however, can be clearly excluded as a source of visible luminescence, as the bound hole energy of $2.5 \mathrm{eV}$ would leave only a luminescence energy between 0.4 and $1.8 \mathrm{eV}$. Even the largest calculated bandgap of $5.4 \mathrm{eV} \mathrm{[48]} \mathrm{is} \mathrm{not} \mathrm{large} \mathrm{enough} \mathrm{to} \mathrm{yield} \mathrm{the} \mathrm{peak} \mathrm{energy} \mathrm{of} 2.6 \mathrm{eV}$.

As mentioned in section 2.2, a $2.5 \mathrm{eV}$ absorption band (observed at $20 \mathrm{~K}$ under $532 \mathrm{~nm}$ picosecond pump pulse excitation) has been assigned to the bound hole polarons and vanishes under heating up to room temperature or under additional $\mathrm{HeNe}$ laser illumination [8,9]. An explanation for this disappearance may be an excitation of the bound hole polarons releasing them as free hole polarons from the defect trap.

The first measurements of this luminescence have been made by Koppitz et al. [16] and Klose et al. [15] and revealed a strong dependence on Mg doping, but also on stoichiometry and temperature. These measurements have been carried out for different stoichiometries and $\mathrm{Mg}$ dopings as well as in a temperature range from room temperature down to $77 \mathrm{~K}$. The samples have been excited with X-rays [16] or with a Xenon lamp as well as with an excimer laser (249 nm, $20 \mathrm{~ns})$ for lifetime measurements [15], respectively. 
a)
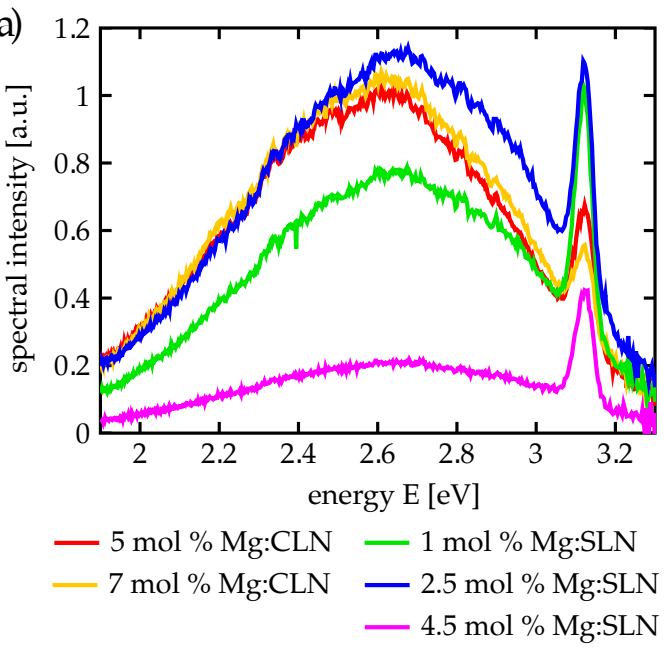

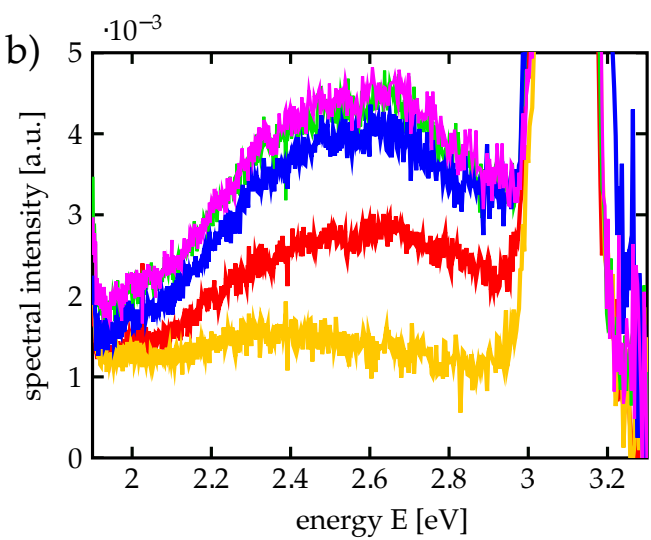

— undoped SLN - $1 \mathrm{~mol} \% \mathrm{Mg}: \mathrm{CLN}$

- undoped CLN $-2 \mathrm{~mol} \% \mathrm{Mg}: \mathrm{CLN}$

$-3 \mathrm{~mol} \% \mathrm{Mg}: \mathrm{CLN}$

c)

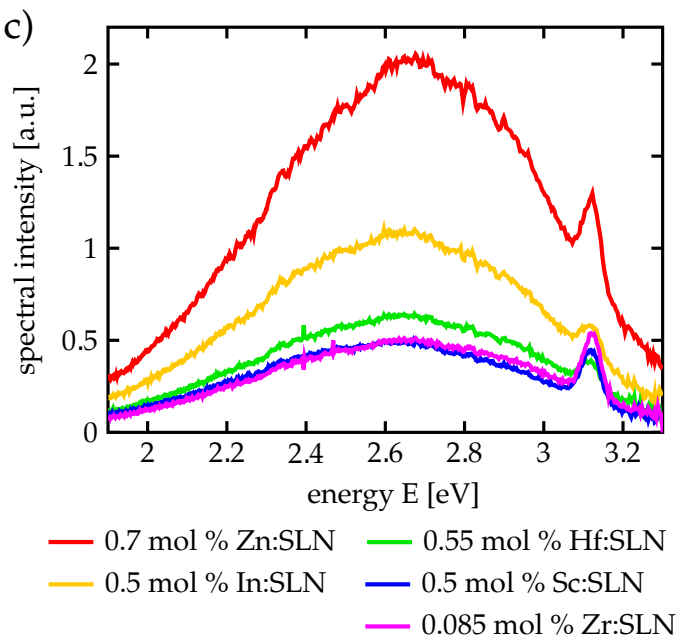

Figure 4. Spectra of the $2.6 \mathrm{eV}$ photoluminescence induced by three-photon femtosecond excitation (100 fs at $790 \mathrm{~nm}, 170 \mathrm{~mW}$ ). (a) Photoluminescence from Mg:CLN and Mg:SLN with different Mg dopings. (b) Photoluminescence from undoped CLN and SLN and weakly Mg doped CLN. (c) Photoluminescence from SLN doped with different optical damage resistant dopants. The strong peak around $3.1 \mathrm{eV}$ in all curves stems from second-harmonic generation. Note that all diagrams $(\mathbf{a}-\mathbf{c})$ are normalized to the same arbitrary units to make all curves comparable. This shows the weakness of the luminescence from the weakly doped samples shown in (b) which amounts to not more than a few $10^{-3}$ units

The following behavior of the luminescence in Mg-doped CLN has been measured:

- At $77 \mathrm{~K}$ a strong increase of the luminescence intensity as a function of the doping concentration has been found. The strongest increase by almost 2 orders of magnitude was between 2 and $4 \mathrm{~mol} \% \mathrm{Mg}$, accompanied by a weaker increase between 4 and $9 \mathrm{~mol} \%$ [15].

- Also measured at $77 \mathrm{~K}$ : the luminescence intensity of $6 \mathrm{~mol} \% \mathrm{Mg}: \mathrm{LNO}$ for a range of different $\mathrm{Li} /(\mathrm{Li}+\mathrm{Nb})$ ratios, showing an increase of $2-3$ orders of magnitude between $42.5 \%$ (strong $\mathrm{Li}$ deficiency) and CLN stoichiometry [15].

- The luminescence is also strongly temperature-dependent [16]. The ratio of luminescence intensities at $80 \mathrm{~K}$ and at $295 \mathrm{~K}$ reaches values of almost 200 for Mg:CLN with dopings of 4 mol $\%$ and higher. This means, at these dopings the luminescence increases by a factor of about 200 when cooling down to $77 \mathrm{~K}$. At low dopings of $2 \mathrm{~mol} \%$ and below, this ratio is quite lower. This low value has not been exactly displayed in the concerning diagram, but seems to be in the order 
of 10 . The increase of this ratio between 2 and $5 \mathrm{~mol} \%$ is clearly related to the doping threshold and the replacement of $\mathrm{Nb}_{\mathrm{Li}}$ anti-sites.

The temporal behavior of the luminescence showed also to be strongly influenced by temperature and doping. Using a pulsed excimer laser $(\lambda=249 \mathrm{~nm})$ with $20 \mathrm{~ns}$ pulses, the lifetimes of the luminescence were measured for different temperatures between 20 and $300 \mathrm{~K}$ and dopings up to $9 \mathrm{~mol} \%$ :

- Undoped CLN showed a constant lifetime of almost $10^{-7} \mathrm{~s}$ at all temperatures.

- At the low temperatures the lifetimes grow with the Mg doping, reaching about $4 \times 10^{-7} \mathrm{~s}$ at $20 \mathrm{~K}$ around the doping threshold.

- From 20 to $200 \mathrm{~K}$ the luminescence lifetimes of the doped samples fall almost linearly, reaching the value of the undoped CLN at $200 \mathrm{~K}$. Between 200 and $300 \mathrm{~K}$ all lifetimes are almost constant at the value of undoped CLN.

It has been assumed that below the doping threshold nonradiative decay channels quench the luminescence, while these channels are suppressed around the doping threshold [15-17]. This behavior of the nonradiative decay channels has been attributed to variations of the electron-phonon interaction [15-17], which appears to be probable as Xin et al. found a moderate reduction of the electron-phonon interaction by $50 \%$ around the doping threshold [42]. As nonradiative decay in a solid is related with the emission of phonons, this reduction will probably affect the luminescence quenching as well. However, a reduction by $50 \%$ is not sufficient at all to explain the huge variations of the luminescence intensity by about two orders of magnitude. On the other hand, the doping and stoichiometry dependencies indicate that the luminescence will be suppressed through the presence of $\mathrm{Nb}_{\mathrm{Li}}$ anti-site defects. In comparison to the moderate variations of the electron-phonon coupling, the complete removal of the $\mathrm{Nb}_{\mathrm{Li}}$ anti-sites is obviously the ultimate cause of the strong luminescence enhancement. This indicates that these intrinsic defects are very important for the non-radiative decay channels. Hence, reducing the defect concentration by doping around the threshold increases the luminescence and its lifetime, while an increase of the lithium deficiency reduces the luminescence intensity again by restoring the $\mathrm{Nb}_{\mathrm{Li}}$ anti-sites [15].

Note that there is still the strong temperature dependence of the luminescence above the doping threshold, indicating the existence of different luminescence-quenching channels being independent from $\mathrm{Nb}_{\mathrm{Li}}$ anti-sites. If such channels are responsible for the luminescence quenching at higher temperatures, they are obviously very sensitive to temperature.

The effect of defect concentration on luminescence has also been reported for the luminescence from dopants as $\mathrm{Cr}^{3+}$ and $\mathrm{Er}^{3+}[85,86]$. Although these cases deal not with polaronic luminescence itself we will discuss them in the next paragraphs because they provide some deeper insights into the luminescence quenching mechanism.

As the luminescence spectra from dopant ions originate from their radiative transitions, this radiation is obviously excited by the transition of electrons from the conduction band (or from neighbored polarons) over unoccupied states of the dopant ion to the valence band-via at least one radiative transition. In principle these radiative transitions can be quenched by the same mechanisms that also quench radiative electron and hole polaron recombinations-via concurring nonradiative channels returning excited charge carriers back to the valence band, bypassing all radiative transitions. The first example are the strong variations of the $\mathrm{Cr}^{3+}$ luminescence in $\mathrm{Cr}$-doped LNO under varying stoichiometry, as there is a strong luminescence quenching in non-stoichiometric LNO depending on the number of defects [85]. A similar example is the enhancement of $\mathrm{Er}^{3+}$ luminescence in Er-doped CLN at higher Mg dopings, as reported by Tang et al. [86]. Absorption measurements showed the presence of bipolarons in these materials and a reduction of this presence through $\mathrm{Mg}$ doping. As the measurements showed no detectable sign of single bound polarons, the authors proposed this concurring decay to be mediated by bipolaron formation and subsequent recombination with hole polarons [86], as depicted in Figure 1. This decay channel depends immediately on the presence of $\mathrm{Nb}_{\mathrm{Li}}$ anti-sites that 
can form bipolarons. It is conceivable that not the whole bipolaron has to be recombined with two hole polarons at once, but only one of the two constituting electron polarons with one hole polaron. This would leave behind the other electron polaron as a bound polaron, where a new free electron polaron can aggregate. At any case, this nonradiative decay would require the presence of $\mathrm{Nb}_{\mathrm{Li}}$ anti-sites as hosts. As these anti-sites are surrounded by lithium vacancies, it is possible that the nonradiative recombination takes place between bipolarons and hole polarons that have been trapped as bound hole polarons in the neighborhood of these lithium vacancies. For the visible polaronic luminescence, this quenching mechanism may explain well the luminescence quenching in LNO samples with a higher amount of $\mathrm{Nb}_{\mathrm{Li}}$, as in CLN samples with low $\mathrm{Mg}$ doping. When at low or zero dopings very much anti-sites are present, the nonradiative recombination might also take place via single bound polarons instead of bipolarons; the important point is again the presence of $\mathrm{Nb}_{\mathrm{Li}}$ anti-sites. Furthermore, it is also possible that the niobium interstitials $\mathrm{Nb}_{\mathrm{V}}$ show physically the same behavior, thus contributing comparably to the nonradiative recombination. The temperature-dependent luminescence quenching occurring in highly doped Mg:CLN at room temperature, however, cannot explained in that way due to the absence of $\mathrm{Nb}_{\mathrm{Li}}$ anti-sites. As already stated above, the reason for that quenching must be found in different concurring nonradiative recombination processes, maybe mediated by Mg-containing defect complexes.

The temperature dependence of luminescence in higher doped CLN has also parallels in the luminescence from dopant ions. For $\mathrm{Tb}^{3+}$ luminescence in $2.8 \mathrm{~mol} \% \mathrm{~Tb}: \mathrm{CLN}$, temperature-dependent quenching was observed that sets in above $150 \mathrm{~K}$. Its temperature dependence shows an Arrhenius-like behavior with an activation energy of $0.22 \mathrm{eV}$ [87]. This value lies within the range of the polaron hopping energy, thus indicating an involvement of polaron hopping processes in the luminescence quenching. Note that a $2.8 \mathrm{~mol} \% \mathrm{~Tb}^{3+}$ doping may approach the doping threshold for $\mathrm{Tb}^{3+}$, leaving only few $\mathrm{Nb}_{\mathrm{Li}}$ anti-sites in the material. In that case we would have an analogy to the temperature-dependent luminescence quenching in Mg:CLN with doping concentrations around the threshold. On the other hand, the temperature-dependent behavior of $\mathrm{Cr}^{3+}$ luminescence in Cr-doped SLN showed to be determined by the electron-phonon coupling. In a range from 77 to $300 \mathrm{~K}$ the temperature dependence of the $\mathrm{Cr}^{3+}$ luminescence agreed well with calculations based on a quantitative model of electron-phonon interaction [88], thus leaving no space for any indications for an involvement of polaron hopping. Probably the nonradiative decay channel exists in the $\mathrm{Cr}^{3+}$ ions themselves and is directly related with the emission of phonons without any energy transfer to polarons.

These examples demonstrate a variety of possible luminescence quenching mechanisms in (doped) LNO. As we will discuss in the next section, the temperature dependence of the visible polaronic luminescence in highly Mg-doped CLN shows also an Arrhenius-like behavior with activation energies in the range of the polaron hopping energy, thus being comparable to the case of $\mathrm{Tb}^{3+}$ luminescence.

Our first investigations on this visible photoluminescence $[17,18]$ mainly dealt with the investigation of its domain contrast to be discussed in the next part. We detected the luminescence spectrum with a maximum around $2.6 \mathrm{eV}$ and a width of almost $0.35 \mathrm{eV}$ upon a three-photon excitation. For that we focused a near-infrared femtosecond laser beam from a Ti:Sa laser (100 fs at $790 \mathrm{~nm}$ and with a repetition rate of $75 \mathrm{MHz}$ ) through a high N.A. immersion objective onto 0.5 to $1 \mathrm{~mm}$ thick z-cut samples. To avoid the pyrolysis of the immersion oil, the focus was kept $10 \mu \mathrm{m}$ inside the sample bulk. To overcome the bandgap, a three-photon process is necessary for this excitation wavelength, as has also been indicated by power-dependent measurements of the luminescence intensity [17]. The luminescent light was collected by the same objective and detected by a spectrometer after being coupled out by a beamsplitter and after blocking of the $790 \mathrm{~nm}$ stray light. We also applied a spectral intensity calibration for both the spectrometer as for the detection path. However, the excitation wavelengths of our pulsed lasers (and their higher harmonics) were too far off the effective wavelengths for the NIR luminescence excitation. Therefore our investigations have been concentrated on the visible luminescence band. 
Our luminescence measurements have confirmed the strong dependence on $\mathrm{Mg}$ doping, especially on the $5 \mathrm{~mol} \%$ doping threshold: For CLN samples of 5 and of $7 \mathrm{~mol} \% \mathrm{Mg}$ doping, respectively, high luminescence intensities have been observed, while for $3 \mathrm{~mol} \%$ and below the luminescence intensity was lower by at least two orders of magnitude, as shown in Figure 4.

For this review we have carried out additional measurements of the luminescence spectrum of stoichiometric lithium niobate (SLN) samples to prove the importance of the doping threshold for different dopings and stoichiometries, too. For this purpose SLN samples have been prepared in the Wigner Research Center for Physics in Budapest, where SLN has already been prepared for investigations on the bandgap [43]. The SLN samples prepared for our luminescence investigations had been doped with different $\mathrm{Mg}$ concentrations as well as with other optical damage resistant dopants like $\mathrm{Zn}$, In, Sc, Hf, and Zr. The dopant concentrations were chosen to be always above the threshold, which depends on the growth process and the valence state of the dopant $[89,90]$. Our aim was to take a further look on the luminescence quenching not only by a different stoichiometry but especially by different dopants than $\mathrm{Mg}$. Note that due to the still non-perfect stoichiometry of usual SLN there is still a finite doping threshold of about $1 \mathrm{~mol} \%$ for $\mathrm{Mg}$ doping [36], while improved approaches to perfect stoichiometry yielded a further reduction of the $\mathrm{Mg}$ doping threshold to as low as about $0.2 \mathrm{~mol} \% \mathrm{Mg}$ [37].

Figure 4a shows the luminescence spectra of Mg:CLN and Mg:SLN. Both 5 and $7 \mathrm{~mol} \% \mathrm{Mg}: C L N$ and 1, 2.5 and $4.5 \mathrm{~mol} \% \mathrm{Mg}: S \mathrm{LN}$ show a luminescence intensity in the same order of magnitude. The decrease in luminescence at $4.5 \mathrm{~mol} \%$ doping in Mg:SLN may be a consequence of an increasing number of defects that far above the doping threshold. Moreover, all samples show a strong second-harmonic peak around $3.1 \mathrm{eV}$.

Figure $4 \mathrm{~b}$ shows the weak three-photon luminescence from undoped CLN and SLN, as well as from CLN doped below the threshold. The luminescence is at least two orders of magnitude smaller than for threshold and super-threshold doping, thus confirming the findings presented above. As the SLN crystals still show a certain Li deficiency, they contain enough luminescence-suppressing $\mathrm{Nb}_{\mathrm{Li}}$ defects to keep the luminescence on the same low level as for the weakly doped Mg:CLN samples. In accordance with our expectations, the luminescence signal from undoped CLN is lowest as these crystals contain the highest concentrations of $\mathrm{Nb}_{\mathrm{Li}}$.

Figure 4c shows photoluminescence spectra of SLN with different damage-resistant dopants: $\mathrm{Hf}, \mathrm{Zr}, \mathrm{Zn}, \mathrm{Sc}$ and In, that all leave the SLN transparent. The luminescence strength is indeed of the same order of magnitude as for the $1 \mathrm{~mol} \% \mathrm{Mg}$-doping around the threshold. The considerable luminescence at dopings of around $0.5 \mathrm{~mol} \%$ for Sc, In and $\mathrm{Hf}$, as well as the stronger luminescence for $0.7 \mathrm{~mol} \% \mathrm{Zn}$, compared to the weak luminescence of undoped SLN as shown in Figure $4 \mathrm{~b}$, confirms the influence of the low threshold of SLN on the luminescence quenching. An outstanding example, however, is the low $\mathrm{Zr}$ doping being accompanied with a luminescence intensity as high as for the $0.5 \mathrm{~mol} \%$ doping of other dopants. Doping SLN with $\mathrm{Zr}$, however, showed indeed a comparably low doping threshold of only $0.085 \mathrm{~mol} \%$ [91] instead of $0.2-1 \mathrm{~mol} \%$ as in the case of $\mathrm{Mg}$ doping [36,37]. Moreover, even for congruent lithium niobate a rather low doping threshold of only $2 \mathrm{~mol} \%$ has been found for $\mathrm{Zr}$ doping [92]. Note that the dopings of $\mathrm{Zn}$ [93] as well as for $\mathrm{Hf}$ and $\mathrm{Zr}$ have been directly measured, while all other concentrations have been determined from the melt. Deviations between the doping concentrations in the melt and in the crystal as well as slightly different doping thresholds may explain e.g., the larger luminescence intensity from $0.7 \mathrm{~mol} \% \mathrm{Zn}$ compared to $1 \mathrm{~mol} \% \mathrm{Mg}$ or of $0.5 \mathrm{~mol} \%$ In compared to $0.5 \mathrm{~mol} \%$ Sc or $0.55 \mathrm{~mol} \% \mathrm{Hf}$.

Thus, we have confirmed that also the doping-induced luminescence enhancement is really independent from the specific properties of $\mathrm{Mg}$ defect ions and works in the same way with different optical damage resistant dopants. Note that all these luminescence spectra show the same shape with the maximum around $2.6 \mathrm{eV}$ and a width of almost $0.35 \mathrm{eV}$, independent from the specific kind of dopant. This underlines again the polaronic origin of that luminescence instead of being caused by any specific dopant defect centers (like the $\mathrm{Er}^{3+}$ luminescence in Er:LNO [94,95]), and it confirms the 
identical mechanism of luminescence enhancements by doping with these dopants which leads to the replacement of $\mathrm{Nb}_{\mathrm{Li}}$ anti-sites.

In addition to these damage-resistant dopings that leave the samples transparent, we have also made measurements with SLN and CLN samples doped with Fe, Mn or Er. These impurities-that change the absorption in the visible range drastically-do not yield any sign of an enhanced $2.6 \mathrm{eV}$ photoluminescence. This is probably due to their strong absorption or by providing new strong decay channels. Only the Er dopants show the known Er luminescence [86,94,95]. Especially Fe does not increase the visible luminescence peak at all; even a sample with all Fe ions being brought into the $\mathrm{Fe}^{3+}$ state (changing the sample color from dark red to pale yellow) did not show any luminescence peak around $2.6 \mathrm{eV}$.

\subsection{Time Dependent Measurements of the Visible Luminescence Band}

To obtain a deeper insight into the nature of the visible photoluminescence we carried out time-dependent measurements that have been published in a separate paper [19]. In these measurements we used a pulsed UV laser light source at $266 \mathrm{~nm}$ with $4 \mathrm{~ns}$ pulses up to $5 \mathrm{~mJ}$. The setup allowed to measure luminescence lifetimes up to the range of ms. Strongly enhanced luminescence signals were obtained by cooling the sample down to a temperature range of 100 to $200 \mathrm{~K}$. The sample surfaces were illuminated under $45^{\circ}$ and the luminescence was detected under $90^{\circ}$ to the exciting beam. Time-averaged luminescence spectra measured from $7 \mathrm{~mol} \% \mathrm{Mg}$ :CLN for different temperatures are shown in Figure 5, showing a strong decrease of the luminescence with temperature. The strong temperature dependence of the luminescence has thus been confirmed for dopings around the threshold, indicating the existence of further luminescence quenching mechanisms not being mediated by $\mathrm{Nb}_{\mathrm{Li}}$ anti-sites.

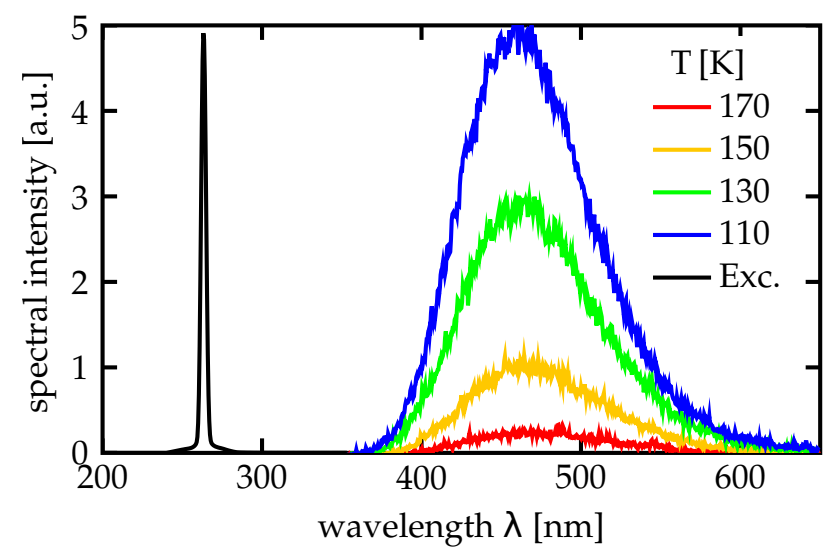

Figure 5. Temperature dependence of the normalized time-averaged visible photoluminescence from $7 \mathrm{~mol} \% \mathrm{Mg}$ :CLN induced by pulsed ns UV laser light at different temperatures. This figure is reproduced from our previous publication T. Kämpfe et al., Phys. Rev. B 2016, 93, 174116 [19].

However, at these deep temperatures a layer of microscopic ice crystals was formed by sublimated water, which affects the reflection properties of the sample surfaces. As this effect increased with lower temperatures the measured intensities could not be used for an exact quantitatively analysis but only for a simple demonstration of the increase of luminescence quenching with temperature. For more precise measurements another setup would be necessary keeping the sample in vacuum or within a completely dry nitrogen atmosphere. Additionally, like with our thee-photon femtosecond setup no near-infrared luminescence has been detected with the UV laser pulse setup.

Consequently, the time-dependent measurements of luminescence have been carried out at $150 \mathrm{~K}$, using the advantage of reduced luminescence suppression and making also measurements at sub-threshold dopings feasible. Like in the case of the non-radiative decay of bound polarons into the Fe defect centers, a stretched-exponential decay of the luminescence can be found. This confirms 
the polaron recombination as the luminescence source, as the recombination of spatially randomly distributed polarons yields a superposition of different exponential decays $[15,19]$. The intensity of the luminescence hence has to obey the equation

$$
I(t)=I_{0} t^{\beta-1} \exp \left[-(t / \tau)^{\beta}\right] .
$$

corresponding to the time-derivative of the stretched-exponential decay of the number of excited charge carriers:

$$
n(t)=n_{0} \exp \left[-(t / \tau)^{\beta}\right],
$$

which is typical for transitions that form an ensemble of emitters with distributed decay rates $[19,71,78]$. The closer $\beta$ to 1 , the nearer the decay comes to a single exponential. A small $\beta$, however, indicates a broader distribution of decay times. After passing of the exciting pulse the relaxation of excited free charge carriers into polarons takes place within picoseconds [50-52], so that at later times only recombining electron and hole polarons are present and their dynamics can be described just by Equation (2).

We carried out our time-dependent measurements at CLN samples with dopings above and below the doping threshold, namely at $3 \mathrm{~mol} \%$ and $7 \mathrm{~mol} \% \mathrm{Mg}$ content, to investigate the behavior in these two regimes. While the visible luminescence of $3 \mathrm{~mol} \% \mathrm{Mg}: \mathrm{CLN}$ is very weak at room temperature, the cooled nanosecond excitation setup provides the possibility to observe the increased luminescence intensities at low temperatures. This follows from the measurements in Ref. [16], showing a strong temperature depence of luminescence already at $3 \mathrm{~mol} \% \mathrm{Mg}$ doping, which allows good luminescence measurements for both dopings at low temperatures.

a)

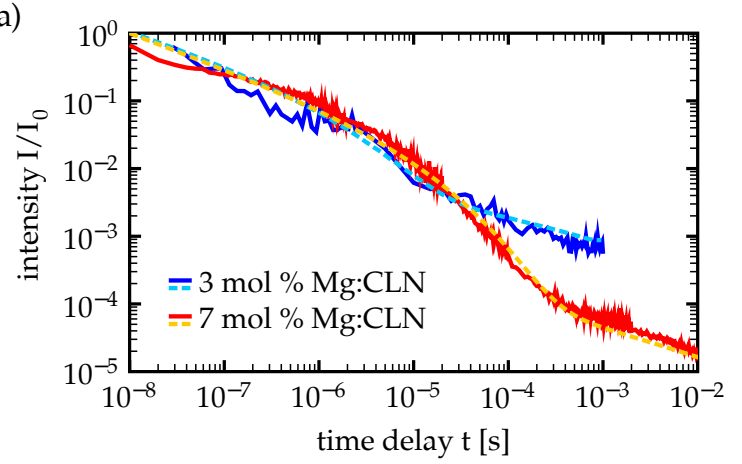

b)

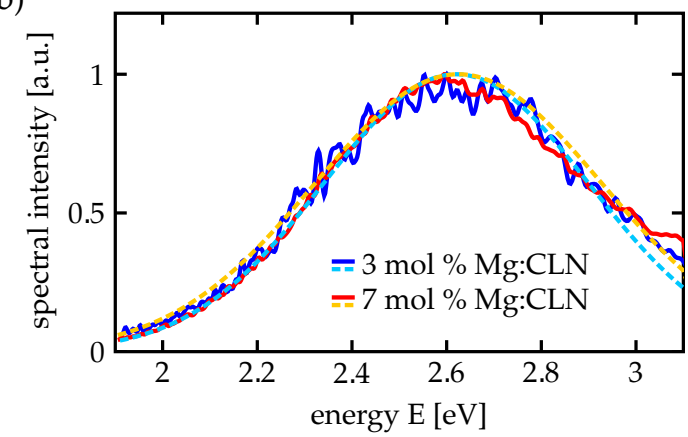

Figure 6. (a) Time-dependent measurements of the photoluminescence from $3 \mathrm{~mol} \% \mathrm{Mg}$ :CLN and $7 \mathrm{~mol} \% \mathrm{Mg}$ :CLN at $150 \mathrm{~K}$, recorded with the ns-pulsed UV laser setup around the spectral maximum. The continuous lines show the measured data while the dashed lines show the stretched-exponential fits. (b) Photoluminescence spectra from the UV nanosecond laser setup, averaged over the first $5 \times 10^{-3} \mathrm{~s}$ after each exciting laser pulse (continuous lines). The peak positions and peaks from Figure 4 are confirmed, as Gaussian fits (dashed lines) of the spectra result in peaks with maxima around $2.6 \mathrm{eV}$ and widths in the range of $0.3 \mathrm{eV}$. The graphs in this figure are reproduced from our previous publication $\mathrm{T}$. Kämpfe et al., Phys. Rev. B 2016, 93, 174116 [19].

In both cases of 3 and $7 \mathrm{~mol} \% \mathrm{Mg}$ doping we found that the temporal decay has to be fitted with a superposition of two stretched exponentials, resulting in each two values $\tau_{1}$, $\tau_{2}$ (with $\tau_{1}$ in the order of $\mu \mathrm{s}$ and $\tau_{2}$ in the order of $10 \mathrm{~ms}$ to $1 \mathrm{~s}$ ) and $\beta_{1}, \beta_{2}$ (between 0.5 and 0.7 ), as shown in Table 1 . The decay curves are displayed double-logarithmically in Figure 6a, corresponding spectra are shown in Figure $6 \mathrm{~b}$, the latter confirming the peak position at $2.6 \mathrm{eV}$ and the width around $0.3 \mathrm{eV}$. For the time-dependent measurements the luminescence spectrum has been integrated over a small range around the spectral maximum. Especially the luminescence decay curve from the $7 \mathrm{~mol} \% \mathrm{Mg}$ sample shows a distinct separation into two regimes below $10^{-4} \mathrm{~s}$ and around $5 \times 10^{-4} \mathrm{~s}$ that have indeed to be 
fitted with two stretched exponentials. $I_{1,2}^{\max }$ are the prefactors of the stretched exponentials and give the contributions of these exponentials to the intensity $I^{\max }$ at $t=0$. The subscript "max" denotes that the measured time-dependent intensity is obtained by integration around the spectral maximum, to which the stretched exponentials are fitted. In Table $1, I_{1}^{\max }$ and $I_{2}^{\max }$ are normalized for each sample to the respective larger value. For $7 \mathrm{~mol} \% \mathrm{Mg}$ they have a small ratio of $I_{2}^{\max } / I_{1}^{\max }$ of about $3.5 \times 10^{-2}$, i.e., the intensity from the first stretched exponential is considerably higher than from the second one. For the $3 \mathrm{~mol} \% \mathrm{Mg}: \mathrm{CLN}$, however, the opposite is the case: the inverse ratio $I_{1}^{\max } / I_{2}^{\max }$ is very small at about $2.3 \times 10^{-3}$ and the first exponential shows a rather weak contribution. As a consequence, the kink between the two regimes is much less pronounced.

Table 1. Characteristic lifetimes $\tau_{1,2}$, stretched-exponential parameters $\beta_{1,2}$, the intensities $I_{1,2}^{\max }$, the peak energies $E_{i}^{\max }$ and widths $w_{i}$ from time-dependent luminescence measurements on $\mathrm{Mg}$-doped $\mathrm{LiNbO}_{3}$ samples, recorded with the nanosecond-pulsed UV laser setup at $T=150 \mathrm{~K}$ as shown in Figure $6 \mathrm{a}$. The index $i$ denotes the first and the second stretched exponential. The intensities $I_{1}^{\max }$ and $I_{2}^{\max }$ of these stretched exponentials on the spectral maximum are normalized for each doping to the larger one. These data are reproduced from our previous publication T. Kämpfe et al., Phys. Rev. B 2016, 93, 174116 [19].

\begin{tabular}{ccccccc}
\hline Mg doping [mol \%] & $\boldsymbol{i}$ & $\boldsymbol{\tau}_{\boldsymbol{i}}[\mathrm{s}]$ & $\boldsymbol{\beta}_{\boldsymbol{i}}$ & $\boldsymbol{I}_{\boldsymbol{i}}^{\mathbf{m a x}}[\mathrm{a.u.}]$ & $\boldsymbol{E}_{\boldsymbol{i}}^{\mathbf{m a x}}[\mathrm{eV}]$ & $\boldsymbol{w}_{\boldsymbol{i}}[\mathrm{eV}]$ \\
\hline 7 & 1 & $(12 \pm 2) \times 10^{-6}$ & $0.48 \pm 0.05$ & 1 & $2.6 \pm 0.1$ & $0.30 \pm 0.03$ \\
& 2 & $(1.1 \pm 0.2) \times 10^{0}$ & $0.59 \pm 0.05$ & $(3.5 \pm 0.5) \times 10^{-2}$ & & \\
3 & 1 & $(2.0 \pm 0.5) \times 10^{-6}$ & $0.52 \pm 0.11$ & $(2.3 \pm 0.3) \times 10^{-3}$ & $2.6 \pm 0.1$ & $0.28 \pm 0.03$ \\
& 2 & $(1.0 \pm 0.5) \times 10^{-2}$ & $0.70 \pm 0.05$ & 1 & & \\
\hline
\end{tabular}

The separation of the time development into two regimes for $7 \mathrm{~mol} \% \mathrm{Mg}$ :CLN may be explained by the time development of hole polaron and electron polaron density. Within some hundred fs after each exciting pulse the excited charge carriers relax into polaron states $[47,50,51]$, and free electron and hole polarons occur in such a density that each charge carrier finds a partner for recombination in its nearest neighborhood. Thus, many recombinations involve none or only few electron/hole polaron hoppings to neighbored sites because adjacent electron and hole polarons can recombine directly. This yields the first stretched exponential as a superposition of shorter lifetimes from direct recombinations about small distances and only few hoppings. These conditions change when these charge carriers - the majority of excited charge carriers-have recombined and the remaining charge carriers have to move over longer distances by hopping. This results in a different superposition of decay times where the contributions from the recombinations are negligible compared to the longer hopping times [19]. These different contributions of direct recombinations and hopping times may explain the existence of two regimes that make two stretched-exponential fits sensible.

For the $3 \mathrm{~mol} \% \mathrm{Mg}: \mathrm{LNO}$, however, the relative intensity of the first exponential is much weaker than of the second. This behavior is probably caused by the stronger nonradiative decay below the doping threshold: Most of the electron and hole polarons created through the exciting pulse recombine nonradiatively, which obviously causes a very strong drain of the first regime.

After these first time-dependent measurements at the $3 \mathrm{~mol} \%$ and the $7 \mathrm{~mol} \% \mathrm{Mg}$ :CLN samples, the measurements on the $7 \mathrm{~mol} \% \mathrm{Mg}$ :CLN sample — the one with the larger luminescence-have been extended to different temperatures between 100 and $200 \mathrm{~K}$. These measurements are shown in Figure $7 \mathrm{a}$, revealing a strong temperature dependence of the decay curves and their parameters.

Figure $7 \mathrm{~b}$ shows the dependence of $\beta_{1}$ and $\beta_{2}$ on the temperature, Figure $7 \mathrm{c}$ the temperature dependence of the lifetimes $\tau_{1}$ and $\tau_{2}$. Although higher temperatures yield a faster recombination and lower decay times, the higher energy of the crystal and its constituents appear to yield a broader distribution of the decay times resulting in stretched exponentials with lower $\beta$ values. Note that the curves from the lower temperatures show the kink which distinctly separates the two regimes with different $\beta^{\prime}$ s and $\tau^{\prime}$ s, while at $200 \mathrm{~K}$ this kink has disappeared. The higher $\beta$ values at the 
lower temperatures render the curves more exponential-like, yielding a stronger bending in the double-logarithmic plot that makes the kink between the two regimes more pronounced. This is related with a narrower distribution of decay times in the two regimes, indicated by the $\beta$ parameters closer to 1. The vanishing of the kink at $200 \mathrm{~K}$, however, coincides with smaller $\beta$ and may indicate that the broadening of these decay time distributions yields an overlap of these distributions that dissolves the clear separation of the two regimes.

Moreover, the ratio $I_{2}^{\max } / I_{1}^{\max }$ of the second exponential intensity to the first showed a strong temperature dependence: at $100 \mathrm{~K}_{2} / I_{1}$ is about $10^{-4}$ while it rises to the factor 10 at $200 \mathrm{~K}$ [19]. Like in the case of $3 \mathrm{~mol} \% \mathrm{Mg}$ :CLN this behavior probably stems from the luminescence quenching that increases with temperature and drains the first regime, thus lowering $I_{1}$ in relation to $I_{2}$. Note that due to the sublimation of water on the cooled sample surface a sensible quantitative evaluation of the absolute intensities $I_{1}$ and $I_{2}$ was not possible, while the division of $I_{2}$ by $I_{1}$ at each temperature cancels this unwanted effect.
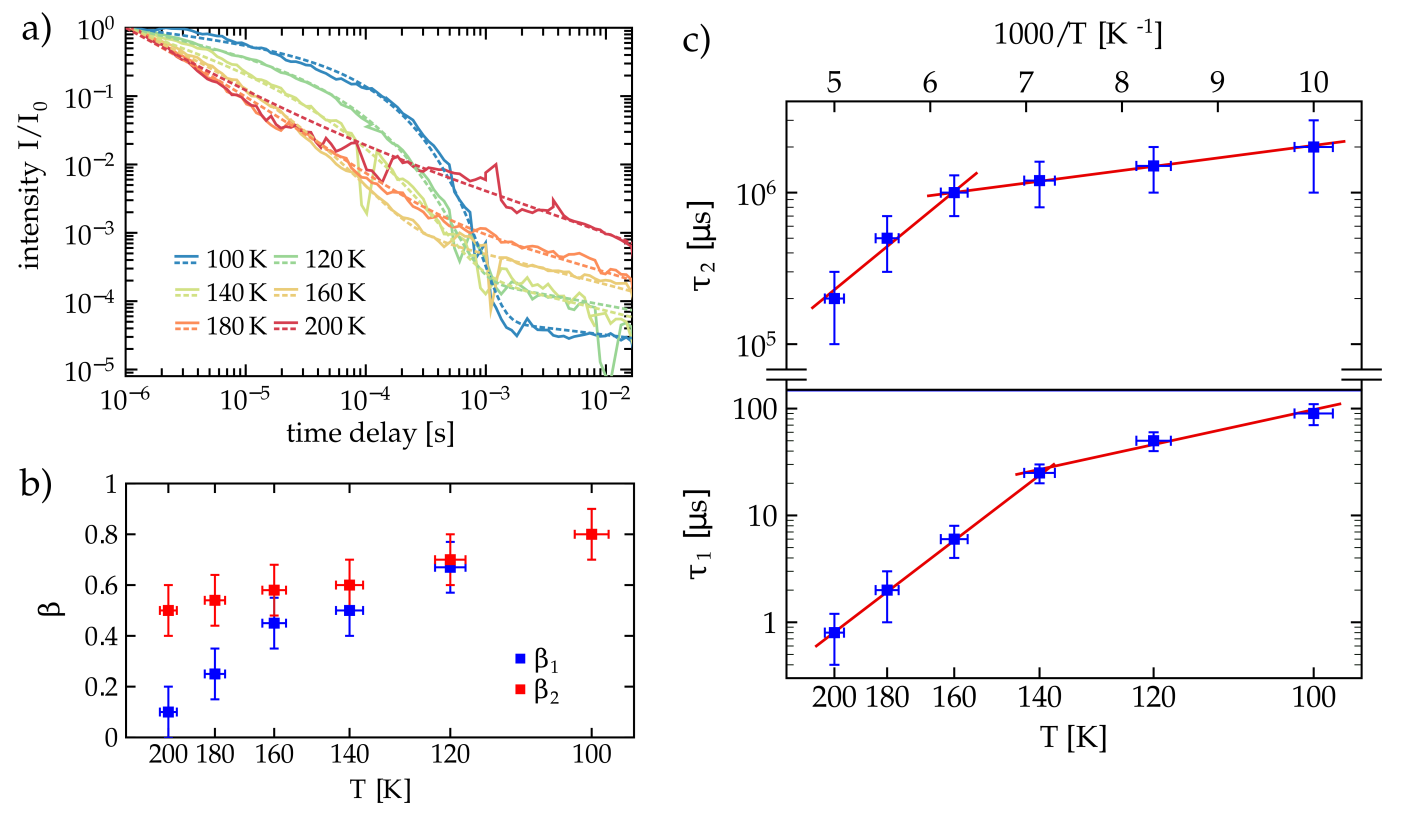

Figure 7. (a) Normalized time-dependent photoluminescence of $7 \mathrm{~mol} \% \mathrm{Mg}$ :CLN for various temperatures (continuous line) and stretched-exponential fits (dashed lines) under nanosecond-pulsed UV illumination. (b) Plots of $\beta_{1}$ and $\beta_{2}$ from these fits over temperature. (c) Arrhenius plots of lifetimes $\tau_{1}$ and $\tau_{2}$ (logarithmic plots over $1 / T$ ). These diagrams are reproduced from our previous publication T. Kämpfe et al., Phys. Rev. B 2016, 93, 174116 [19]; for this publication, new fits have been made for all parts of the curves in (c).

The luminescence lifetimes $\tau_{1}$ and $\tau_{2}$, shown in Figure $7 \mathrm{c}$, drop by 1-2 orders of magnitude from 100 to $200 \mathrm{~K}$. They show an Arrhenius-like behavior with two distinct regimes below and above $150 \mathrm{~K}$. Using the Arrhenius-like law

$$
\tau(T)=\tau_{0} \exp \left[E_{\mathrm{a}} / k T\right],
$$

the activation energies $E_{\mathrm{a}}$ can be obtained through linear fits by plotting the logarithm of $\tau$ over $1 / T$ (Arrhenius plot). In that kind of plot two regimes with constant slopes appear. All resulting activation energies are shown in Table 2. Fitting the lifetimes $\tau$ in the higher temperature range between $150 \mathrm{~K}$ to $200 \mathrm{~K}$ yields activation energies of $(139 \pm 22) \mathrm{meV}$ for $\tau_{1}$ and $(103 \pm 48)$ for $\tau_{2}$. For temperatures below $150 \mathrm{~K}$, however, the fits yield much lower activation energies of $\tau_{1}$ and $\tau_{2}$. 
Table 2. Activation energies $E_{\mathrm{a}}$ obtained from the Arrhenius plots of the temperature-dependent lifetimes $\tau_{1}$ and $\tau_{2}$ as shown in Figure $7 \mathrm{c}$. From each $\tau_{1}$ and $\tau_{2}$ the two different linear regimes above and below 140 or $160 \mathrm{~K}$, respectively, have been fitted.

\begin{tabular}{ccc}
\hline Lifetime & Temperature Range & $\boldsymbol{E}_{\boldsymbol{a}}[\mathrm{meV}]$ \\
\hline$\tau_{1}$ & $>140 \mathrm{~K}$ & $139 \pm 22$ \\
& $<140 \mathrm{~K}$ & $38 \pm 10$ \\
$\tau_{2}$ & $>160 \mathrm{~K}$ & $103 \pm 48$ \\
& $<160 \mathrm{~K}$ & $16 \pm 16$ \\
\hline
\end{tabular}

These results raise the question about the origin of this Arrhenius-like behavior with these activation energies. For a possible answer we will discuss now a hypothetical picture. Note that this picture is based on the assumption that the bandgap energy is around $4 \mathrm{eV}$, yielding a rather low free hole polaron energy around $0.3 \mathrm{eV}$. This low energy is important for the following discussion. A higher bandgap and a higher free hole polaron energy, however, would result a completely different picture. Despite this ambiguity we present the following considerations as an example in what manner these recombination processes could be modeled.

If only a radiative recombination would occur, the radiative lifetime $\tau_{r}$ would be determined by the mobilities of free electron and hole polarons. Assuming the considerably lower free polaron energy of $0.3 \mathrm{eV}$ would also yield a stronger delocalization, resulting into a lower activation energy and a higher mobility. Due to their higher mobility the recombination dynamics is dominated by the dynamics of the free hole polarons, because the hole polarons move faster and do not have to wait for the slower free electron polarons. The temperature dependence of the radiative recombination lifetime $\tau_{\mathrm{r}}$ should then be determined by the low hole polaron activation energy. With real electron polaron hopping energies in a range of $0.1-0.24 \mathrm{eV}[58,64,65]$ associated to an electron polaron energy of $1 \mathrm{eV}$, the hopping energies of hole polarons (with $0.3 \mathrm{eV}$ polaron enery) can accordingly be estimated to be in a range of $0.03-0.08 \mathrm{eV}$. The activation energy of $\tau_{1}$ for $T<140 \mathrm{~K}$-almost $40 \mathrm{meV}$-lies at the lower end of that range. This is consistent with the assumption that the radiative recombination is dominant in the range between 100 and $150 \mathrm{~K}$.

Around $150 \mathrm{~K}$, however, another regime with an activation energy of $139 \mathrm{meV}$ appears. As demonstrated by Figure 5, the luminescence intensity drops also strongly with the temperature, indicating a concurring nonradiative recombination. As the luminescence intensity has dropped by more than two orders of magnitude at $200 \mathrm{~K}$, this process can be considered as dominant for $T>150 \mathrm{~K}$ and the higher activation energy can be assigned to this nonradiative recombination. This activation energy fits well with the measured activation energies obtained from electrical DC conductivity $[4,58,64,65]$, indicating that the nonradiative recombination process is mediated by free polaron hopping. The activation energies of $\tau_{2}$ are smaller than for $\tau_{1}$, but $\tau_{2}$ behaves still quite similar to $\tau_{1}$.

As there are no $\mathrm{Nb}_{\mathrm{Li}}$ anti-sites in $7 \mathrm{~mol} \% \mathrm{Mg}: \mathrm{CLN}$, the nonradiative recombination cannot be mediated by bipolarons (or bound polarons). As there are still lithium vacancies $\mathrm{V}_{\mathrm{Li}}$ in the substrate, especially as parts of the $\mathrm{Mg}_{\mathrm{Li}}+\mathrm{V}_{\mathrm{Li}}$ defect clusters, the recombination of free polarons with bound hole polarons may be a promising candidate for a nonradiative recombination channel. As the bound hole polarons are trapped at the lithium vacancies, the recombination dynamics would be governed by the hopping of the free electron polarons that have to meet these bound holes, thus resulting an activation energy according to the electron polaron hopping energy. Thus, the $\mathrm{Mg}_{\mathrm{Li}}+\mathrm{V}_{\mathrm{Li}}$ defect clusters would, in a certain sense, take over the role of the $\mathrm{Nb}_{\mathrm{Li}}$ anti-sites in undoped or weakly doped LNO. However, in Mg-doped LNO also $\mathrm{Mg}_{\mathrm{V}}$ interstitials that occupy regularly empty octahedra, including associated lithium vacancies, would probably contribute to luminescence quenching as well by providing similar nonradiative recombination channels. In this hypothetical model, the mechanisms of hole polarons by lithium vacancies would be the same. 
A similar behavior of the temperature-dependent quenching of $\mathrm{Tb}^{3+}$ luminescence has already been discussed in the last section [87]. The activation energy of $0.22 \mathrm{eV}$ matching to polaron hopping has been found at temperatures above $150 \mathrm{~K}$, at lower temperatures the luminescence was almost constant. This indicates that the $\mathrm{Tb}^{3+}$ luminescence is quenched by the same nonradiative recombination channel. Unfortunately we were not able to round the picture off by an exact evaluation of the luminescence intensities over different temperatures.

We note again that this speculative model requires a low free hole polaron energy and high mobility, based on a bandgap of $4 \mathrm{eV}$. As the question of the exact bandgap value being relevant for the photoluminescence is not yet finally resolved, proper modeling of the recombination physics requires a reliable knowledge of the energy and other properties of free hole polarons. These data should be obtained by independent measurements.

A comparison with the lifetime measurements made by Klose et al. [15] reveals some further unclear points. Note that the presented lifetime measurements are made with similar nanosecond-pulsed laser light (20 ns pulses at $249 \mathrm{~nm}$ in the measurements of Klose et al. and $4 \mathrm{~ns}$ at $266 \mathrm{~nm}$ in our measurements). Klose et al. did not differentiate between $\tau_{1}$ and $\tau_{2}$; as they measured lifetimes in the range below $10^{-6} \mathrm{~s}$ it is sensible to compare their measurements with our $\tau_{1}$ values. When we can thus compare the lifetime measurements by Klose et al. with our $\tau_{1}$ values, the discussion of the results shows that the following points deserve a deeper investigation:

- Doping CLN with $\mathrm{Mg}$ reduces the luminescence quenching induced by $\mathrm{Nb}_{\mathrm{Li}}$ and increases the lifetimes [15]. This indicates that the radiative recombination and the nonradiative recombination via $\mathrm{Nb}_{\mathrm{Li}}$ live indeed from the same reservoir of (freely moving) polarons, because the presence of the additional nonradiative recombination reduces the total lifetime of $n(t)$ and therefore also the luminescence lifetime. While the replacement of $\mathrm{Nb}_{\mathrm{Li}}$ by $\mathrm{Mg}$ doping increases the lifetime, however, the temperature-dependent quenching at the higher dopings brings the lifetime back just to the value at zero doping. Is that accordance only accidentally or based on deeper reasons? The answer to this question requires a deeper understanding of the radiative and nonradiative recombination processes.

- At zero Mg doping the lifetime measured by Klose et al. remained constant between 100 and $300 \mathrm{~K}$. The strong luminescence suppression without doping underlines the dominance of the nonradiative recombination mediated by the $\mathrm{Nb}_{\mathrm{Li}}$ anti-sites. Obviously the dynamics of this recombination is rather insensitive to temperature.

However, it seems to be difficult to find a consistent explanation for that. When the nonradiative recombination process probably lives on the same polaron reservoir like the radiative recombination, it is difficult to understand why not at least the hole polaron activation energy should occur in the luminescence lifetime, yielding at least a temperature sensitivity as in $7 \mathrm{~mol} \%$ Mg:CLN below $150 \mathrm{~K}$.

Although the nonlinear recombination via bipolarons or bound polarons at $\mathrm{Nb}_{\mathrm{Li}}$ sites looks very probable, a clear model of this recombination, that can easily explain the lifetime behavior, is still missing.

- In these lifetime measurements the luminescence lifetimes decrease roughly linearly in the temperature range up to $200 \mathrm{~K}$. Plotting $\tau_{1}$ from Figure $7 \mathrm{c}$ not logarithmically, but in a linear scale over $T$, it also decreases roughly linearly up to $160 \mathrm{~K}$ and nestles to the $T$ axis at higher temperatures. However, this nestling occurs already at 160 , and not at $200 \mathrm{~K}$. Moreover, $\tau_{1}$ covers a range from almost 100 to about $1 \mu \mathrm{s}$, being reduced to about $1 / 100$ of the value at $T=100 \mathrm{~K}$. The lifetimes from Klose et al., on the other hand, are in the order of not more than $5 \mu \mathrm{s}$, and around $200 \mathrm{~K}$ they stay constant at still $1 / 5$ of the maximum at $80 \mathrm{~K}$.

We could not explain these differences as in both measurement series Mg-doped CLN and similar kinds of laser light have been used. Future investigations should therefore also take a look if and how further parameters take stronger influence on the luminescence dynamics. Especially the influence of excitation intensity and pulse energy should be checked. 
- At all, the lifetime measurements of $\tau_{1}$ and $\tau_{2}$ should be expanded to measurement series from undoped CLN to $7 \mathrm{~mol} \% \mathrm{Mg}$ :CLN in steps of $1 \mathrm{~mol} \% \mathrm{Mg}$ doping. Furthermore, not only different dopings but also stoichiometries are probably interesting for investigations. Especially the behavior of the activation energies and the temperature of transition between the different regimes (with different activation energies) would be of interest.

Moreover, clearer measurements of the temperature dependence of the luminescence intensities $I_{1}^{\max }$ and $I_{2}^{\max }$ are necessary for a sensible modeling of the recombination processes. These measurements should, of course, cover the same range of $\mathrm{Mg}$ dopings and stoichiometries.

- The discussed model includes the influence of the temperature-dependent polaron hopping. However, nonradiative transition processes in solids require the emission of phonons (or, in general, similar neutral quasiparticles as e.g. magnons). Thus a thorough understanding of the temperature dependence of the radiative and nonradiative recombination rates for different dopings comprises necessarily the knowledge of the temperature and doping dependence of the electron-phonon coupling as well. An interesting question may be if the electron-phonon coupling contributes significantly to the strong increase of the temperature dependence of luminescence around the doping threshold. On the one hand, the doping dependence of this interaction, as given by Xin et al. [42], is too moderate to explain the large doping dependence of the luminescence. On the other hand, the influence of electron-phonon coupling on the temperature dependence of luminescence is unclear. The discussed Arrhenius plots of the lifetimes did not allow to separate the influence of electron-phonon coupling from the influence of polaron hopping. To separate the influence of the electron-phonon coupling onto the luminescence from other processes like polaron hopping, not only further lifetime measurement series but also additional investigations with different methods are necessary.

- The electron-phonon coupling is possibly not the only further influence that has to be taken into consideration. As shortly mentioned above, the polaron model breaks down at sufficient deep temperatures due to a transition to an itinerant polaron band [66-69]. However, we have not found preciser information on this temperature range for LNO. In the relevant temperature range, this transition would probably have a great impact on the luminescence and its dynamics.

To conclude this discussion, the nonradiative recombination via bipolarons/bound electron polarons at $\mathrm{Nb}_{\mathrm{Li}}$ and via bound hole polarons at $\mathrm{Mg}_{\mathrm{Li}}+\mathrm{V}_{\mathrm{Li}}$ defect clusters may provide good models for the luminescence quenching through low doping or at higher temperatures. To really understand the radiative and nonradiative recombination dynamics much more and deeper investigations are necessary. These investigations will allow to prove the proposed theories; if they are successful, the models could be developed towards an accurate agreement with the measured data.

The time-and temperature dependent luminescence measurements on 3 and $7 \mathrm{~mol} \% \mathrm{Mg}: C L N$, that have been thoroughly discussed in this section, have already been published in Ref. [19]. For this review, further time-dependent measurements have been carried out with the same set of SLN samples that we already used to extend our results on luminescence spectra, together with $5 \mathrm{~mol} \% \mathrm{Mg}: \mathrm{CLN}$ for comparison. We recorded the time-dependent luminescence now at $100 \mathrm{~K}$, the results are shown in Figure 8 , the $\beta$ values and lifetimes from these measurements are presented in Table 3.

The lifetimes were mostly in the order of $10^{-5}-10^{-6} \mathrm{~s}$ for $\tau_{1}$ and $0.01 \mathrm{~s}$ for $\tau_{2}$, while most $\beta_{1}$ values lie between 0.4 and 0.8 and most $\beta_{2}$ in the range of 1 . However, fitting of the second (long-time) stretched exponential turned out to be difficult in many of these cases, hence the corresponding $\tau_{2}$ and $\beta_{2}$ values suffer from larger uncertainties. The differences between these values for different dopings indicate a significant influence of the dopant on the luminescence decay. Most outstanding is the behavior of the $4.5 \mathrm{~mol} \% \mathrm{Mg}: \mathrm{SLN}$ sample with strongly reduced $\tau_{2}$ and $\beta_{2}$. This corresponds to the behavior of the curve for times $>10^{-3}$ s showing a much less distinct kink and a much stronger negative slope compared to the other curves, resulting in an extraordinary small $\tau_{2}$. It seems that doping far above the SLN doping threshold may cause certain degrees disorder in the crystal structure with significant impacts on the temporal behavior of luminescence. 
a)

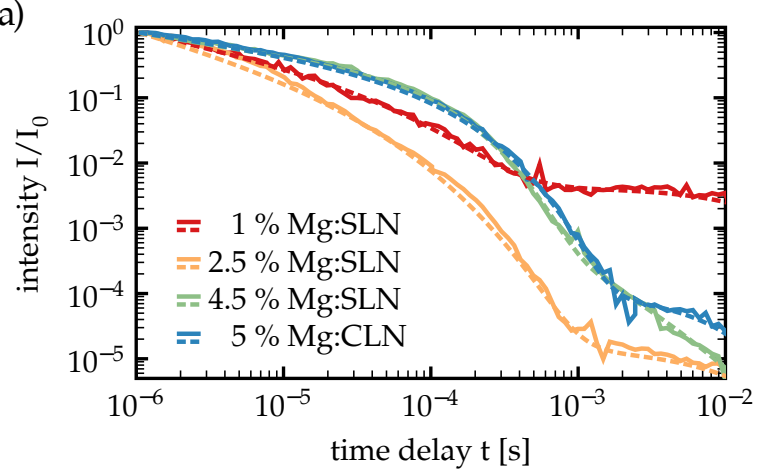

b)

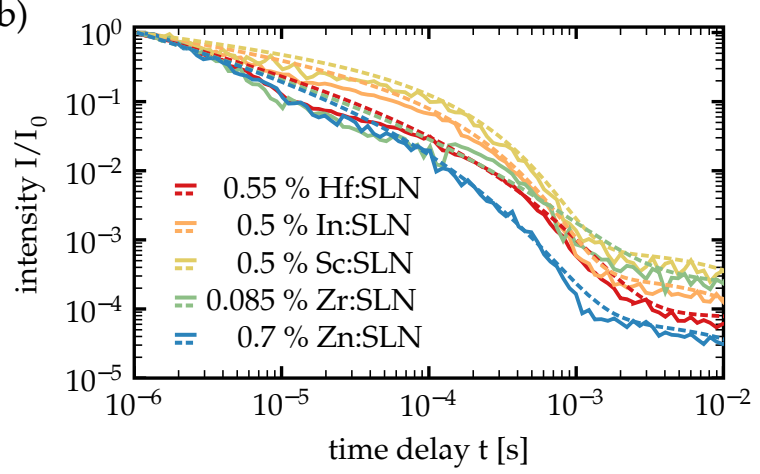

Figure 8. Normalized time-dependent photoluminescence from SLN recorded with the nanosecond UV laser setup at $100 \mathrm{~K}$ : (a) Time-dependent photoluminescence from Mg:CLN and Mg:SLN (b) Time-dependent photoluminescence from SLN doped with other dopants.

Table 3. Characteristic lifetimes $\tau_{1,2}$ and stretched-exponential parameters $\beta_{1,2}$ for doped SLN samples and for $5 \mathrm{~mol} \% \mathrm{Mg}: \mathrm{CLN}$ at $T=100 \mathrm{~K}$ as fitted from the time-dependent photoluminescence measurements shown in Figure 8. The asterisk * added to the doping number denotes the only CLN sample in this series. The relative intensities are taken from the maxima of the luminescence curves in Figure 4 and are normalized to the intensity from $5 \mathrm{~mol} \% \mathrm{Mg}$ :CLN.

\begin{tabular}{ccccccc}
\hline Dopant & Doping $[\mathbf{m o l} \% \mathbf{~}$ & $\mathbf{I} / \mathbf{I}_{\mathbf{5 ~} \mathbf{~ m o l}} \% \mathbf{~ M g}$ & $\tau_{\mathbf{1}}\left[\mathbf{1 0 ^ { - 5 }} \mathbf{s}\right]$ & $\tau_{\mathbf{2}}\left[\mathbf{1 0} \mathbf{-}^{-\mathbf{2}} \mathbf{s}\right]$ & $\boldsymbol{\beta}_{\mathbf{1}}$ & $\boldsymbol{\beta}_{\mathbf{2}}$ \\
\hline & $5^{*}$ & 1.00 & $9.0 \pm 0.2$ & $0.70 \pm 0.03$ & $0.68 \pm 0.07$ & $1.0 \pm 0.1$ \\
$\mathrm{Mg}$ & 1 & 1.05 & $4.0 \pm 0.2$ & $1.9 \pm 0.6$ & $0.55 \pm 0.02$ & $1.0 \pm 0.1$ \\
& 2.5 & 0.77 & $1.0 \pm 0.1$ & $1.2 \pm 0.2$ & $0.46 \pm 0.20$ & $0.9 \pm 0.2$ \\
& 4.5 & 0.21 & $8.0 \pm 0.3$ & $0.005 \pm 0.004$ & $0.78 \pm 0.02$ & $0.27 \pm 0.03$ \\
\hline $\mathrm{Zr}$ & 0.085 & 0.50 & $10 \pm 4$ & $6.0 \pm 6.7$ & $0.41 \pm 0.5$ & $1.0 \pm 1.5$ \\
$\mathrm{Sc}$ & 0.5 & 0.50 & $12.0 \pm 0.3$ & $1.5 \pm 0.5$ & $0.73 \pm 0.1$ & $1.0 \pm 0.1$ \\
$\mathrm{In}$ & 0.5 & 1.08 & $9.0 \pm 0.3$ & $1.3 \pm 0.3$ & $0.66 \pm 0.01$ & $1.0 \pm 0.2$ \\
$\mathrm{Hf}$ & 0.55 & 0.63 & $7.0 \pm 0.5$ & $11.0 \pm 7.5$ & $0.46 \pm 0.2$ & $1.0 \pm 0.1$ \\
$\mathrm{Zn}$ & 0.7 & 2.02 & $3.0 \pm 0.2$ & $1.7 \pm 1.0$ & $0.45 \pm 0.02$ & $1.0 \pm 0.1$ \\
\hline
\end{tabular}

\subsection{Summary of Part 2}

In LNO the existence of electron and hole polarons has been proven. Excited electrons can occur

1. in free polaron states on $\mathrm{Nb}_{\mathrm{Nb}}$ sites with an absorption maximum at $1 \mathrm{eV}$,

2. in bound polaron states on $\mathrm{Nb}_{\mathrm{Li}}$ anti-sites with an absorption maximum at $1.6 \mathrm{eV}$,

3. or in bound pairs of bound and free electron polarons, called bipolarons, with an absorption maximum at $2.5 \mathrm{eV}$.

To obtain the absorption spectra of these electron polarons, electrons have been brought into these polaron states by reduction of the crystal or by optical excitation of $\mathrm{Fe}^{2+}$-doped samples. In this way the bound polaron $(1.6 \mathrm{eV})$ and bipolaron $(2.5 \mathrm{eV})$ have been found as well as the dissociation of bipolarons with $0.37 \mathrm{eV}$ dissociation energy and the free electron polarons (around $1 \mathrm{eV}$ ) in samples with strongly reduced numbers of $\mathrm{Nb}_{\mathrm{Li}}$ by $\mathrm{Mg}$ doping.

The hole polarons, however, have been found to occur at oxygen sites. In contrast to free hole polarons the oxygen sites of bound hole polarons adjoin immediately to defects, probably lithium vacancies $[2,9,11]$. The absorption maximum of bound holes has been determined to $2.5 \mathrm{eV}[8,9]$. There is no similar data available on free holes. Due to the uncertainty of the LNO bandgap, the estimation of their energy based on the luminescence peak energy extends still over a larger range from 0.3 to $1.7 \mathrm{eV}$. The exact energy value, however, is important for the properties of hole polarons, as a small energy 
would be related with low activation energies, strong delocalization and high mobility. For a deeper understanding of the recombination processes, the energy level and other properties of the hole polarons should be determined precisely by independent experiments.

Two important photoluminescence bands have been revealed that are mediated by these polaron states: one in the near-infrared between 1 and $2 \mathrm{eV}$ and another in the visible range around $2.6 \mathrm{eV}$. In the near-infrared actually two peaks have been observed: one at $1.5 \mathrm{eV}$ and one at $1.3 \mathrm{eV}$. On the latter peak unfortunately no deeper physical investigations have been conducted so far. The $1.5 \mathrm{eV}$ luminescence peak, however, shows a mono-exponential relaxation, indicating its origin from a single center without transitions between different, spatially randomly distributed defect states. This luminescence has been assigned to the radiative relaxation of an excited state $\left(\mathrm{Nb}_{\mathrm{Li}}^{4+}\right)^{*}$ of the bound polaron. The following properties support that interpretation: The intensity depends on the stoichiometry of the sample that determines the number of $\mathrm{Nb}_{\mathrm{Li}}$ anti-sites and also on the number of $\mathrm{Fe}^{2+}$ ions from which electrons can be excited into a bound polaron state. It further strongly depends on temperature, likely due to a non-radiative decay channel that involves thermally induced free electron polaron hopping on neighboring sites.

In contrast, the visible luminescence at $2.6 \mathrm{eV}$ originates from the recombination of free polarons and free hole polarons. This can be concluded from the typical recombination lifetimes in the $\mu$ s range and from the stretched-exponential luminescence decay being characteristic for the recombination of spatially randomly separated charge carriers. The luminescence and their lifetimes show a strong dependence on temperature, and the luminescence is considerably increased at low temperatures below $200 \mathrm{~K}$. The luminescence increases also strongly with Mg doping concentration up to the doping threshold. The likewise reduction of luminescence by reducing the Li content indicates the obvious importance of the number of $\mathrm{Nb}_{\mathrm{Li}}$ for creating luminescence-quenching non-radiative decay channels. The measurements using SLN doped with various optical damage resistant ions confirm the role of the doping threshold, as can be seen from the small luminescence intensities from undoped SLN and weakly doped CLN, while dopings around the low SLN threshold yield as large luminescence intensities as for the much higher doped $5 \mathrm{~mol} \% \mathrm{Mg}$ :CLN. The occurrence of this enhancement in SLN for different dopants verifies that the mechanism of luminescence enhancement does not depend on the specific properties of $\mathrm{Mg}$, although luminescence intensities, lifetimes, and doping thresholds vary for the differently doped SLN samples. A promising model for the luminescence quenching at low dopings is the mediation of the non-radiative decay by the formation of bipolarons (as proposed for $\mathrm{Er}^{3+}$-doped CLN [86]) or bound polarons. This would explain the requirement of $\mathrm{Nb}_{\mathrm{Li}}$ anti-sites for the nonradiative recombination.

Furthermore, especially at higher $\mathrm{Mg}$ dopings above the threshold, a temperature-dependent luminescence quenching appears. By this quenching the luminescence intensity at room temperature is by about two orders lower than at $100 \mathrm{~K}$ and below. As almost all $\mathrm{Nb}_{\mathrm{Li}}$ anti-sites are replaced around the doping threshold, while $\mathrm{Mg}_{\mathrm{Li}}+\mathrm{V}_{\mathrm{Li}}$ defect clusters appear, obviously another concurring nonradiative recombination process occurs. A probable hypothesis is a nonradiative recombination with bound hole polarons being trapped at the lithium vacancies. However, a full and thorough understanding and modeling of all recombination processes is still missing-especially concerning the temperature dependence of intensities and lifetimes, and the influences from polaron hopping, electron-phonon coupling or further processes. More clarity is also needed about the contributions to luminescence quenching from $\mathrm{Mg}$ or $\mathrm{Nb}$ insterstitials not occupying Li sites but regularly empty oxygen octahedra. We have already suggested that they play probably a similar role like their pendants on Li sites, however, this has still to be confirmed. To solve all these questions, many further investigations would have to be carried out.

\section{Ferroelectric Domain Contrast of the Visible Luminescence Band in Mg:LNO}

After reviewing the various polaron states and the luminescence phenomena based thereon we discuss now the most interesting feature that we have observed with the visible polaronic luminescence. 
The visible photoluminescence turned out to be sensitive to a certain structural property of the crystal bulk, namely imperfections of the electric poling due to the presence of defect complexes $[17,18]$. This sensitivity yields a slight reduction of the luminescence in electrically inverted domains. Up to now, it is not clear if the near-infrared luminescence is also affected by electrical poling, as we did neither observe it with our setups nor other researchers have been published anything about.

In Section 3.1 we give an overview on several known effects of imperfect electric poling on electrical and optical properties of ferroelectrics. We point out especially the $\mathrm{Nb}_{\mathrm{Li}}+4 \mathrm{~V}_{\mathrm{Li}}$ model of polar defect complexes by Kim et al. that is widely accepted to explain these effects in lithium niobate $[28,29]$. In the following Sections 3.2 and 3.3 we focus onto the details of the domain contrast of the visible luminescence band between virgin and poled LNO substrates. In Sections 3.4 we discuss the thermally activated decay of the luminescence domain contrast, its activation energy and physical consequences highlighting the role of lithium vacancies in the thermally induced reorientation of the $\mathrm{Nb}_{\mathrm{Li}}+4 \mathrm{~V}_{\mathrm{Li}}$ defect complexes [29].

\subsection{Imperfect Ferroelectric Switching and Its Influence on Physical Parameters of LNO}

Throughout poling, certain dipolar defect complexes do not follow the inversion of the ferroelectric polarization and end up in a frustrated state after poling. Interactions between the defect dipole and the surrounding inverted crystal structure yield a higher energy of this frustrated state compared to the state before poling. Due to these imperfections on the microscopic scale the electric domain inversion cannot be represented by a simple mirroring of the whole crystal structure or its rotation by $180^{\circ}$. These frustrated states are responsible for differences in the physical properties of the virgin and inverted crystal $[17,18,22,28,29,95]$. The most important electric property affected by this imperfect poling is the coercive field strength. The imperfect poling results in an asymmetry of the coercive fields in forward and reverse directions, which can be expressed in terms of an "internal field" $E_{\text {int }}=\left(\left|E_{\mathrm{f}}\right|---\left|E_{\mathrm{r}}\right|\right) / 2[24,25]$. In the used convention the coercive field in forward direction $E_{\mathrm{f}}$ refers to switching from the original virgin state to a singly inverted state, while the field $E_{\mathrm{r}}$ means switching in the reverse direction - which does not necessarily mean a full restoration of the initial state but a return to the initial direction of ferroelectric polarization. In these terms a positive $E_{\text {int }}$ means that $\left|E_{\mathrm{f}}\right|>\left|E_{\mathrm{r}}\right|$, i.e., switching back to the original orientation of polarization is easier than the inversion starting from the initial state. When the internal field is caused by dipolar defect complexes that does not follow electric inversion, switching into a state with frustrated dipoles needs indeed more effort than the reverse switching. Gopalan et al. observed internal fields of about $5 \mathrm{kV} / \mathrm{mm}$ for (undoped) congruent lithium tantalate (CLT) and about $3.3 \mathrm{kV} / \mathrm{mm}$ for CLN [24-27], and already explained it by the influence of polar defect complexes $[24,28]$.

The question about the defect dipoles behind the internal field has been answered by Kim et al. for lithium tantalate. They proposed the $\mathrm{Ta}_{\mathrm{Li}}+4 \mathrm{~V}_{\mathrm{Li}}$ defect complex as being responsible for this phenomenon [29]. Accordingly $\mathrm{Nb}_{\mathrm{Li}}+4 \mathrm{~V}_{\mathrm{Li}}$ was assigned to the internal field of $\mathrm{LNO}[22,28]$. A strong indication for the involvement of $\mathrm{Nb}_{\mathrm{Li}}$ and $\mathrm{V}_{\mathrm{Li}}$ defects is the increase of the internal field of the $\mathrm{LNO}$ substrate with its deviation from perfect stoichiometry-i.e., the more $\mathrm{Nb}_{\mathrm{Li}}$ defects occur, the stronger are the effects of the according defect structures on domain inversion. Indeed, a linear relation between the internal field and stoichiometry has already been observed $[27,28]$. It is probable that not only the $\mathrm{Nb}_{\mathrm{Li}}$-based defect clusters, but also defect clusters consisting of niobium interstitials $\mathrm{Nb}_{\mathrm{V}}[33,34]$ and associated lithium vacancies [34] contribute to the internal field as well. Like the $\mathrm{Nb}_{\mathrm{Li}}+4 \mathrm{~V}_{\mathrm{Li}}$ clusters the $\mathrm{Nb}_{\mathrm{V}}$-based clusters can expected to have an occurrence proportional to stoichiometry, too.

The luminescence domain contrasts, however, have been measured in LNO doped around the doping threshold, therefore lacking the $\mathrm{Nb}_{\mathrm{Li}}$-based defect clusters, while the $\mathrm{Mg}_{\mathrm{Li}}+\mathrm{V}_{\mathrm{Li}}$ take their place. As the $\mathrm{Mg}_{\mathrm{Li}}+\mathrm{V}_{\mathrm{Li}}$ defect complexes are also dipolar, they will probably go over into frustrated states during electric inversion as well. It is possible that $\mathrm{Mg}_{\mathrm{V}}$ interstitials form, together with lithium vacancies, a further species of $\mathrm{Mg}$-based defect dipoles that show a very similar physical behavior. Being thus responsible for imperfect switching, all these $\mathrm{Mg}$-based defect dipoles can be assumed to 
play a major role for the luminescence domain contrasts. How this can be possible will be discussed in the next section.

Figure 9 illustrates these defect complexes in the ferroelectric substrate. $T$ he $\mathrm{Nb}_{\mathrm{Li}}+4 \mathrm{~V}_{\mathrm{Li}}$ complex has a polar structure with a non-zero dipole moment $\mathbf{p}$, because it consists of three lithium vacancies adjacent to the niobium anti-site and a fourth lithium vacancy displaced in the polar $\mathrm{z}$ direction, as shown in Figure 9a. The defect energy is minimal when the dipole moment is aligned with the ferroelectric polarization $\mathbf{P}$. Figure $9 \mathrm{~b}$ shows the same case for the $\mathrm{Mg}_{\mathrm{Li}}+\mathrm{V}_{\mathrm{Li}}$ dipole. Figure $9 \mathrm{C}$ shows then the situation after an electric domain inversion that does not change the orientation of the defect complex, resulting in a frustrated state with $\mathbf{p}$ antiparallel to $\mathbf{P}$. This situation is energetically unfavorable and therefore has little probability to occur in the virgin crystal. It is the presence of these frustrated states that constitutes the imperfect poling and that influences physical properties as coercive field strengths or luminescence. Figure $9 d$ then shows a thermally induced reorientation of the defect dipole itself, as will be discussed in the next paragraphs.

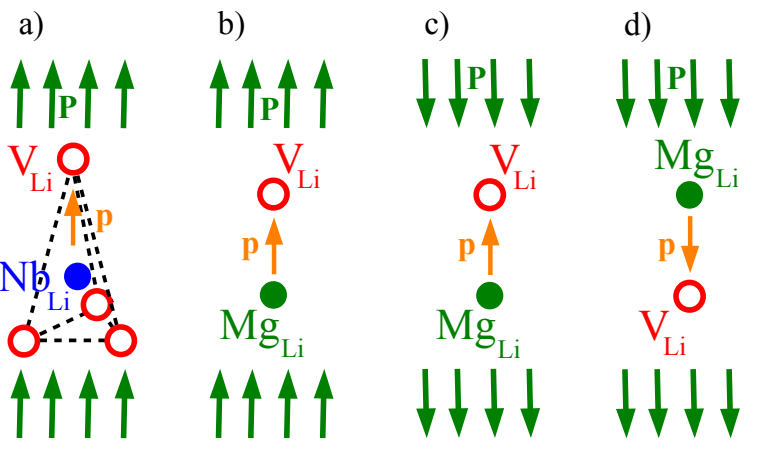

Figure 9. Sketch of defect clusters in virgin and inverted substrate. (a) In undoped $\mathrm{CLN} \mathrm{Nb} \mathrm{Li}_{\mathrm{Li}}+4 \mathrm{~V}_{\mathrm{Li}}$ occur and are obviously responsible for the internal field effect $[28,29]$. The sketch shows the arrangement of the $\mathrm{Li}$ vacancies around the $\mathrm{Nb}_{\mathrm{Li}}$ anti-site. The resulting dipole moment $\mathbf{p}$ shows toward the single Li vacancy on the top due to its larger distance to the rest of the defect complex. In virgin state the dipole moment $\mathbf{p}$ is aligned with the ferroelectric polarization P. (b) In Mg-doped CLN defect dipoles as $\mathrm{Mg}_{\mathrm{Li}}+\mathrm{V}_{\mathrm{Li}}$ occur. In the virgin state, they are also aligned with the ferroelectric polarization $\mathbf{P}$. (c) Inversion of the ferroelectric polarization $\mathbf{P}$ results into a frustrated state with a higher energy. (d) Thermal relaxation of the frustrated state by reorientation of the defect complex is realized when the Li vacancy has thermally moved around the $\mathrm{Mg}_{\mathrm{Li}}$ anti-site.

Further investigations showed a strong influence of annealing on the internal field in a temperature range up to $200-300{ }^{\circ} \mathrm{C}$. Note that in this temperature range no processes like reduction occur that alter the crystal structure chemically. The internal field dropped and even changed its sign when the sample was annealed at different temperatures after a first switching $[24,26]$ - while simple annealing of a virgin sample just preserves the virgin state. This phenomenon expresses a kind of thermal relaxation of the frustrated states. However, monitoring the influence of single switching and subsequent annealing on the internal field requires $E_{\text {int }}$ to be determined from the coercive fields of inversions after annealing. So the original definition of $E_{\text {int }}$ is not useful because it includes the coercive field from the first (pre-annealing) inversion. Gopalan et al. used a modified definition that we will call here $E_{\text {int }}^{*}=\left(\left|E_{\mathrm{f} 2}\right|---\left|E_{\mathrm{r} 1}\right|\right) / 2$ which uses the second inversion in forward direction $\left(E_{\mathrm{f} 2}\right)$ that follows after the first reverse poling $\left(E_{\mathrm{r} 1}\right)$. In that case both inversions used for the definition take place after the annealing that follows after the first inversion.

Figure 10 shows measurements by Gopalan et al. of the internal field $E_{\text {int }}^{*}$ in LNO. Around annealing temperatures of $100^{\circ} \mathrm{C} E_{\text {int }}^{*}$ has been found to drop, and at $150-200^{\circ} \mathrm{C}$ it arrives at a stable negative value with a few smaller absolute value than its positive initial value [26].

Without annealing, reverse inversion is easier than forward inversion. Annealing at higher temperatures after the first switching, however, reverses this relation: forward switching becomes 
easier than backward switching. Thus, annealing after first inversion at temperatures around $150{ }^{\circ} \mathrm{C}$ causes a realignment of the internal field itself.

These measurements confirm that by annealing of the material these defect complexes reconfigure into the opposite orientation. This reorientation is probably realized by a rearrangement of the lithium vacancies due to the thermal mobility of the $\mathrm{Li}$ ions. This explanation is the most probably, not only because the dipolar structure surrounding the $\mathrm{Nb}_{\mathrm{Li}}$ consists of lithium vacancies, but also due to the relatively high thermal mobility of the small lithium ions. Thus, the defect complexes leave the frustrated state and minimize their energy by restoring locally the (by $180^{\circ}$ rotated) virgin state. This realignment is displayed by the realigned internal field [26]. Figure $9 \mathrm{~d}$ displays this result for a $\mathrm{Mg}_{\mathrm{Li}}+\mathrm{V}_{\mathrm{Li}}$ defect dipole. In principle, however, the process is the same also for the $\mathrm{Nb}_{\mathrm{Li}}+4 \mathrm{~V}_{\mathrm{Li}}$ defect complexes: While the $\mathrm{Nb}_{\mathrm{Li}}$ or $\mathrm{Mg}_{\mathrm{Li}}$ anti-sites remain at rest, respectively, the $\mathrm{Li}$ vacancies move around them resulting in a rotated defect complex.

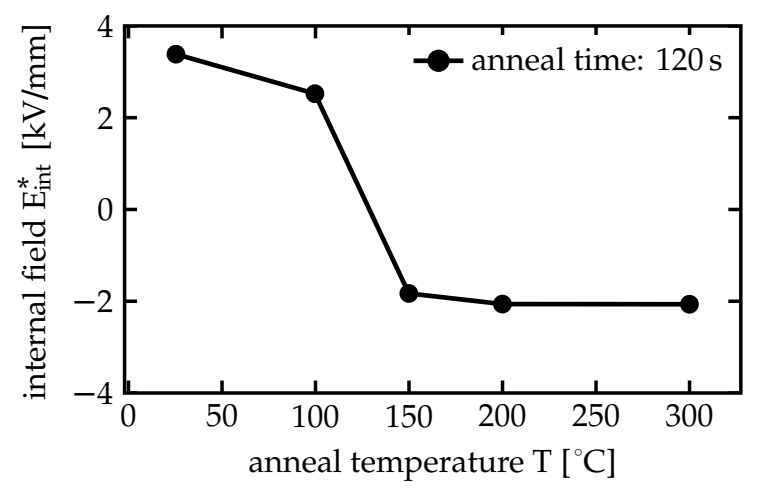

Figure 10. Decay of the internal field $E_{\text {int }}^{*}$ of a CLN crystal under annealing at rising temperatures, measured by Gopalan et al. [26]. The times of heating up before and cooling down after the annealing time of $2 \mathrm{~min}$ did not take more than a few seconds. This figure is reproduced with permission from $\mathrm{V}$. Gopalan et al., Ferroelectrics 1997, 198, 49 [26], copyright (c) 1997 by Taylor \& Francis Ltd.

This interpretation is supported by the observations of the infrared absorption peak of $\mathrm{OH}^{-}$defect ions that is obviously affected by the imperfect poling due to the frustrated polar defect complexes. By inversion this peak shifted in the order of $10 \mathrm{~cm}^{-1}$, and subsequent annealing reversed this shift $[25,26]$.

However, the reversed internal field does not reach again its initial absolute value (Figure 10). This indicates that the $\mathrm{Nb}_{\mathrm{Li}}+4 \mathrm{~V}_{\mathrm{Li}}$ defect structure is not the only species of defect complexes that contribute to the imperfect poling effect. Probably there are also contributions from the $5 \mathrm{Nb}_{\mathrm{Li}}+4 \mathrm{~V}_{\mathrm{Nb}}$ defect complexes based on $\mathrm{Nb}$ vacancies [22,30]. These structures based on the larger $\mathrm{Nb}$ ions cannot be thermally realigned at temperatures up to $200{ }^{\circ} \mathrm{C}$ due to the higher hopping energies of these ions.

In addition to this peculiar electric switching behavior, imperfect inversion can affect several different physical characteristics, especially optical properties. The influence on luminescence is an important point, but also modifications in Raman scattering have been observed. Local Raman scattering measurements showed line shifts on the domain boundaries (domain wall contrast, to be used for monitoring the crystal structure modifications on the domain wall itself) as well as line shifts between inverted domain and virgin surrounding (domain contrast, to be used for monitoring imperfect switching) [20-23].

The influence of ferroelectric poling on luminescence phenomena has also already been observed in several ferroelectric materials doped with luminescent ions. Jia et al. found an influence of the ratio $x$ on the $\mathrm{Eu}^{3+}$ luminescence in Eu-doped $\mathrm{K}_{1-x} \mathrm{Na}_{x} \mathrm{NbO}_{3}$ [96], demonstrating the strong influence of modifications of the crystal structure on the luminescence of embedded defects. An enhancement of the $\mathrm{Pr}^{3+}$ luminescence in a Pr-doped $\left(\mathrm{Bi}_{0.5} \mathrm{Na}_{0.5}\right) \mathrm{TiO}_{3}$ sample by up to $30 \%$ after poling was observed by 
Tian et al. [97]. This enhancement has been explained by a poling-induced lattice distortion in the crystal structure that influences the non-radiative decay. It has also been found that the photoluminescence increases with applied voltage, demonstrating that such luminescent defect ions can act as local probes for the local crystal field [97]. Dierolf et al. investigated Er-doped CLN and SLN and found a domain contrast for intensity and peak positions of the $\mathrm{Er}^{3+}$ luminescence, while the peak positions showed an additional domain wall contrast, too $[23,94,95]$. These effects have been explained by the interaction of polar defect complexes (that may also include the $\mathrm{Er}^{3+}$ ions themselves) with the surrounding crystal. The domain contrasts vanish after annealing at $250{ }^{\circ} \mathrm{C}$ which has also been explained by a thermally induced reorientation of the polar defect dipoles [95].

\subsection{Domain Contrast of Multiphoton Induced Visible Luminescence Band}

In our experiments, however, we have demonstrated that the polaron-mediated luminescence in LNO and LTO shows pronounced domain contrasts as well. In analogy to the internal field, this domain contrast decays under annealing at temperatures between 100 and $200{ }^{\circ} \mathrm{C}$ [17-19]. In this section, we discuss the domain contrasts of the visible $2.6 \mathrm{eV}$ luminescence in different samples, as being measured by three-photon excitation $[17,18]$. These measurements are supplemented by comparison of the time-dependent behavior of virgin and poled substrates using the nanosecond-pulsed UV laser setup [19].

Only few details are known about the details of the mechanism of this luminescence domain contrast. Considerations by Dierolf et al. on the origin of the domain contrast of the $\mathrm{Er}^{3+}$ luminescence involve an influence of the crystal field from frustrated defect complexes (in the neighborhood of $\mathrm{Er}^{3+}$ or containing it) that affects the transition probabilities of these luminescent ions [95], Such mechanisms, however, apply to localized luminescence centers, but not to luminescence by polaron recombination. For the latter, the following variants are conceivable:

1. the defect complexes have a negative or positive influence on the radiative luminescent recombination of electron and hole polarons. The frustrated defect disturb it stronger or or support it to a lesser extent, respectively.

2. the defect complexes have a positive or negative influence on the nonradiative recombination. The frustrated defect dipoles either support it stronger or they disturb it to a lesser extent, respectively.

If the nonradiative recombination in Mg:CLN (doped around the threshold) is mediated by bound hole polarons, these hole polarons would be bound to the lithium vacancies that occur largely in $\mathrm{Mg}_{\mathrm{Li}}+\mathrm{V}_{\mathrm{Li}}$ defect dipoles (and also in the defect dipoles consisting of magnesium interstitials $\mathrm{Mg}_{\mathrm{V}}$ and lithium vacancies). In that case an influence on the nonradiative decay would be more probable than a direct influence on the radiative recombination, as the former is located directly at the dipoles while the latter can take place anywhere. Then the domain contrast may have the following cause: Due to their different influence on the crystal field and modified local potentials, the frustrated defect dipoles may show an increased ability to pull and trap electron polarons to the defects where the recombination takes place. It is also possible that, instead, the trapping of free to bound hole polarons is slightly increased. This would yield a little increased throughput of nonradiative recombination and therefore a slightly stronger luminescence quenching.

The following measurements on the domain contrast have been carried out using the multiphoton excitation setup described above, with the focused femtosecond beam (100 fs, $75 \mathrm{MHz}$ repetition rate) at an average power of $170 \mathrm{~mW}$ and at a wavelength of $790 \mathrm{~nm}$. During all measurements the beam was still focused $10 \mu \mathrm{m}$ into the sample bulk in order to prevent pyrolytical reactions of the used immersion oil. We have obtained the total photoluminescence signal by integrating the luminescence spectrum from 420 to $650 \mathrm{~nm}$. The samples with thicknesses of 0.5 and $1 \mathrm{~mm}$ have been electrically poled at room temperature using water electrodes with O-ring insulation and electric fields up to $20 \mathrm{kV} / \mathrm{mm}[17,18,65,98]$. This poling procedure results in domains with sizes up to several ten microns 
that have grown randomly under the applied voltage. The luminescence domain contrasts have been determined from luminescence scan images of these domains. The images have been obtained by moving the samples with piezo elements horizontally through the exciting femtosecond focus while recording the integrated luminescence signal. The scanning images have been evaluated by averaging the luminescence intensity $I_{\text {virgin }}$ over the brighter virgin domains as well as the intensity $I_{\text {poled }}$ over the darker poled domains. Furthermore, their statistical errors have been extracted. From these values we have obtained the domain contrast and its statistical error by using the definition of the contrast

$$
C=\frac{I_{\text {virgin }}-I_{\text {poled }}}{I_{\text {virgin }}+I_{\text {poled }}}
$$

The resulting domain contrasts, as shown in Table 4, are in the range of a few percent for most of the Mg-doped CLN and SLN samples, while larger values appear for $4.5 \% \mathrm{Mg}$ and for other dopants in SLN. No contrast values are presented for undoped LNO and the sub-threshold CLN samples due to the weak integrated luminescence signal. Note that the generation of stable inverted domains in the used SLN samples had not been possible with all dopants.

Table 4. Domain contrast values of visible photoluminescence for different materials, dopants and doping concentrations recorded with the femtosecond laser setup (790 nm, $170 \mathrm{~mW}$ ). The note " $2 \times$ " behind the doping number denotes a Mg:CLN sample with doubly inverted domains in a singly inverted surrounding. The data for CLN are reproduced with permission from P. Reichenbach et al., Appl. Phys. Lett. 2014, 105, 122906 [18], copyright (C) 2014 by AIP Publishing LLC.

\begin{tabular}{cccc}
\hline Material & Dopant & Doping [mol \%] & Contrast [\%] \\
\hline \multirow{3}{*}{ CLN } & \multirow{2}{*}{$\mathrm{Mg}$} & 5 & $3.7 \pm 0.5$ \\
& & 7 & $2.5 \pm 0.5$ \\
& & 1 & $2.7 \pm 0.5$ \\
\multirow{3}{*}{ SLN } & $\mathrm{Mg}$ & 2.5 & $1.7 \pm 0.6$ \\
& & 4.5 & $2.8 \pm 1.1$ \\
& $\mathrm{Zr}$ & 0.085 & $4.1 \pm 1.5$ \\
\hline \multirow{3}{*}{ SLN } & $\mathrm{In}$ & 0.5 & $8.6 \pm 0.5$ \\
& $\mathrm{Hf}$ & 0.55 & $6.8 \pm 1.0$ \\
\hline
\end{tabular}

The three-photon excitation has the advantage of being able to deeply penetrate the LNO samples due to their high transparency in the near-infrared. Furthermore, the effective focus size is smaller due to the nonlinear-optical absorption, which yields a better resolution. Thus, domain imaging is possible in large depths of the substrate. In the next section such measurements of irregular domains in highly Mg-doped CLT will be presented.

Figure 11 shows some typical multiphoton luminescence scan images of $5 \mathrm{~mol} \% \mathrm{Mg}$ :CLN. Figure 11a shows singly inverted domains, while Figure $11 \mathrm{~b}$ shows doubly inverted domains in a singly inverted surrounding. All these domains show the hexagonal shape that is typical for LNO. The twofold inverted domains appear brighter than the singly inverted surrounding, indicating that they are indeed in a state very close to the virgin state. However, the virgin state cannot be completely restored by twofold inversion, as seen from the reduction of the domain contrast from the initial value of $(3.7 \pm 0.5) \%$ to $(2.5 \pm 0.5) \%$. Obviously not only the forward switching is imperfect, but also the reverse switching cannot completely restore the initial state. The simple picture that inversion just mirrors the crystal structure without the defect dipoles is therefore not realistic either. It is well known that repeated inversion causes a degradation of the crystal structure that also yields a reduction of the internal field as calculated from the $n$-th coercive fields $E_{\mathrm{fn}}$ and $E_{\mathrm{rn}}$.

After determining the domain contrasts using the three-photon excitation by femtosecond pulses, we have also used the UV nanosecond pulse setup in order to take a look on the influence of inversion 
on the stretched-exponential decay. Indeed a comparison of virgin and inverted samples revealed a significant difference in the $\beta_{1}$ parameters. In the poled domains of $3 \mathrm{~mol} \% \mathrm{Mg}$ :CLN and $7 \mathrm{~mol} \%$ Mg:CLN $\beta_{1}$ was smaller than in the respective virgin substrates [19], which corresponds to a slightly faster decreasing luminescence signal. The measured curves are shown in Figure 12. In our previous paper Ref. [19] only $\beta_{1}$ could be evaluated unambiguously. The $\beta_{1}$ values, however, can be obtained much simpler by a linear fit of the double-logarithmic plot. Using $I(t)$ from Equation (1), the function $f(x)=\ln \left[I\left(e^{x}\right)\right]$ has, for small $e^{x}$, the derivative $\beta_{1}---1+O\left(e^{x} / \tau_{1}\right)$, allowing an easy determination of $\beta_{1}$ for $e^{x}=t \ll \tau_{1}$. For this review the $\beta_{1}$ values have thus been newly fitted and are presented in Table 5 . The influence of randomly distributed frustrated dipolar defect complexes on the recombination obviously causes a broadening of the decay time distributions. The coincidence of the reduced $\beta$ with the reduced luminescence can be seen from Equations (1) and (2), as a smaller $\beta$ results in a slower decay of the polaron density $n(t)$ and therefore a smaller and faster decaying luminescence rate $\mathrm{d} n(t) / \mathrm{d} t$.
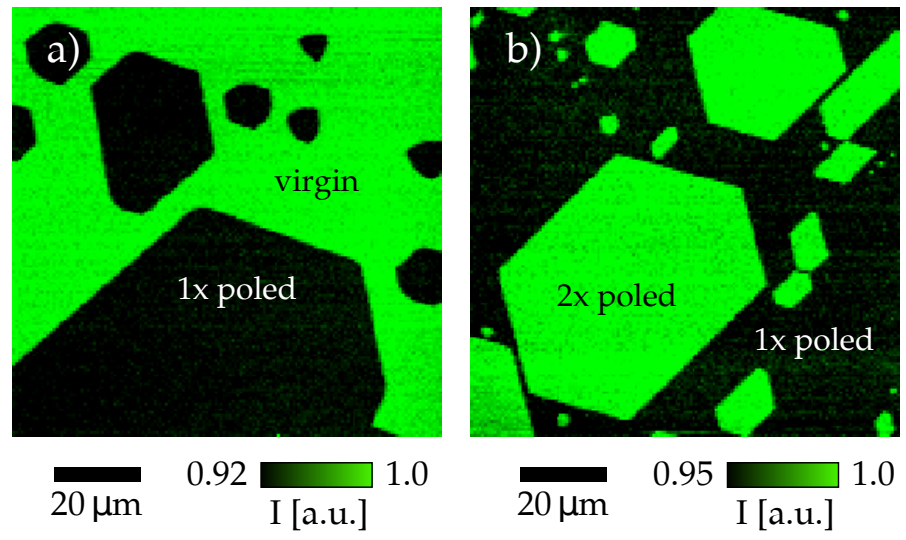

Figure 11. Photoluminescence scan images from poled domains in CLN using the three-photon femtosecond excited visible photoluminescence. (a) Domains within virgin surrounding in $5 \mathrm{~mol} \%$ Mg:CLN. (b) Doubly switched domains within a singly switched area of $5 \mathrm{~mol} \% \mathrm{Mg}$ :CLN, the domains appear now brighter. These figures are reproduced with permission from P. Reichenbach et al., J. Appl. Phys. 2014, 115, 213509 [17], copyright (C) 2014 by AIP Publishing LLC.

a)

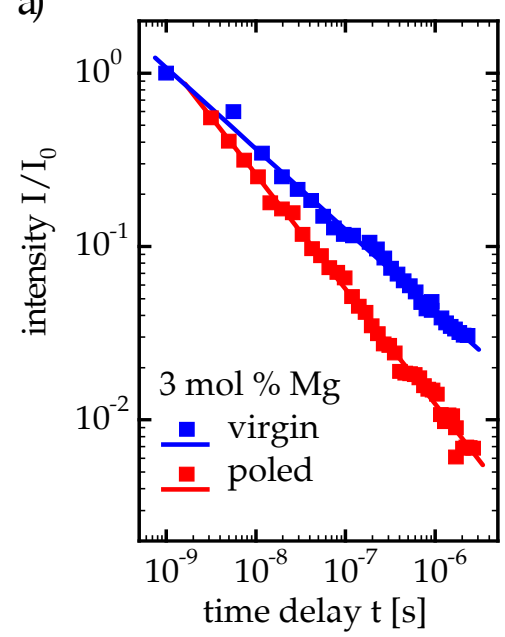

b)

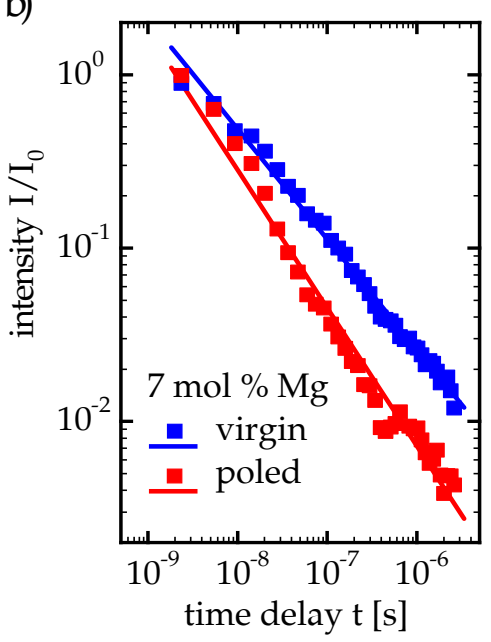

Figure 12. Normalized temporal decay of $2.6 \mathrm{eV}$ photoluminescence in virgin and in poled Mg:CLN at $130{ }^{\circ} \mathrm{C}$, under UV nanosecond-pulsed illumination, and with dopings of $7 \mathrm{~mol} \%$ (a) and $3 \mathrm{~mol} \%$ (b). These figures are reproduced from our previous publication T. Kämpfe et al., Phys. Rev. B 2016, 93, 174116 [19]. 
Table 5. Values of $\beta_{1}$ as obtained from the linear fits of the time-dependent luminescence curves shown in Figure 12, measured at virgin and poled CLN samples with 3 and $7 \mathrm{~mol} \% \mathrm{Mg}$ doping.

\begin{tabular}{ccc}
\hline Mg Doping [mol \%] & $\beta_{\mathbf{1}}$ (Virgin) & $\boldsymbol{\beta}_{\mathbf{1}}$ (Poled) \\
\hline 3 & $0.36 \pm 0.01$ & $0.20 \pm 0.02$ \\
7 & $0.53 \pm 0.01$ & $0.34 \pm 0.01$ \\
\hline
\end{tabular}

\subsection{Excitation Intensity Dependence}

Before turning into the discussion of the contrast decay under annealing we want to point out the important observation that the measured domain contrasts depend strongly on the excitation intensity. In all measurements shown in Sections 3.1, 3.2 and 3.4 the samples were excited with 100 fs pulses at the same average power of $170 \mathrm{~mW}$ and the central wavelength of $790 \mathrm{~nm}$. Varying the power, however, resulted also in variations of the measured domain contrasts. Thus, for consistent contrast measurement series the excitation power should be kept constant. Figure 13a shows the power-dependent domain contrasts for $5 \mathrm{~mol} \% \mathrm{Mg}$ :CLN, $2.5 \mathrm{~mol} \% \mathrm{Mg}: S L N, 0.085 \mathrm{~mol} \% \mathrm{Zr}$ :SLN and also for $8 \mathrm{~mol} \% \mathrm{Mg}$ :CLT. Although congruent lithium tantalate (CLT) will be discussed in the fourth part, we include the CLT data for this special case already here in order to keep the presentation compact. For all these materials a reduction of the domain contrasts was observed with increasing powers, although the decrease is not significant for $2.5 \mathrm{~mol} \% \mathrm{Mg}: S L N$. This means that the absolute luminescence reduction in poled domains is not proportional to the luminescence itself. With increasing powers this difference grows slower than the increasing luminescence, leading to reduced domain contrasts.

a)

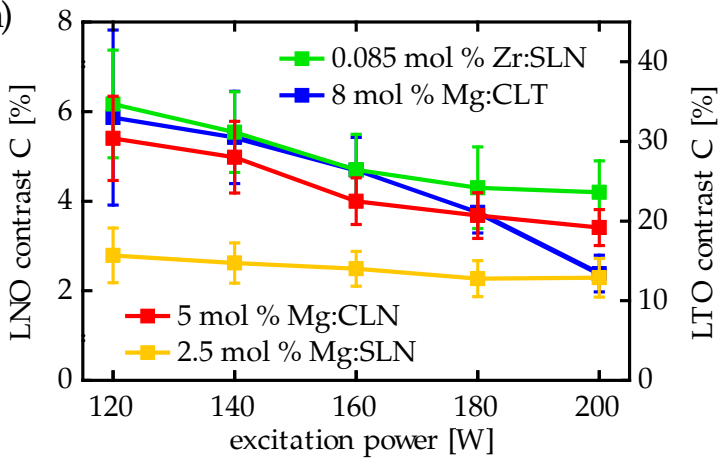

b)

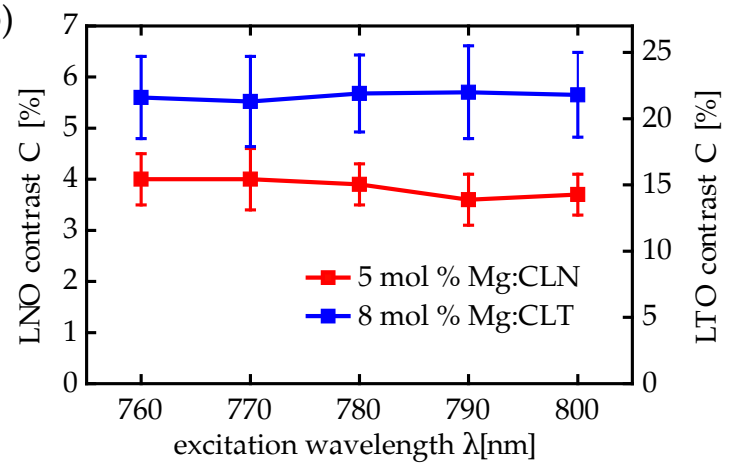

Figure 13. (a) Power dependence of domain contrasts for $5 \mathrm{~mol} \% \mathrm{Mg}$ :CLN, $2.5 \mathrm{~mol} \% \mathrm{Mg}: S L N$, $0.085 \mathrm{~mol} \% \mathrm{Zr}: S L N$ and $8 \mathrm{~mol} \% \mathrm{Mg}: C L T$, recorded with three-photon femtosecond excitation at $790 \mathrm{~nm}$. (b) Excitation wavelength dependence of domain contrasts of $5 \mathrm{~mol} \% \mathrm{Mg}: \mathrm{CLN}$ and $8 \mathrm{~mol} \%$ Mg:CLT with total emitted photoluminescence intensity kept constant.

The origin of that behavior may be a saturation of the domain contrast mechanism. When we adopt the hypothesis that the luminescence is quenched by nonradiative recombination via bound hole polarons, and that the frustrated dipoles as hosts of these polarons catalyze this reaction, an increase of the excitation rate may lead to a saturation of these reaction centers that are responsible for the domain contrast. However, this remains speculative as long as the real physical processes behind the domain contrast are not yet experimentally confirmed.

For $5 \mathrm{~mol} \% \mathrm{Mg}$ :CLN and $8 \mathrm{~mol} \% \mathrm{Mg}$ :CLT, domain contrasts have also been measured for different excitation wavelengths by tuning the central wavelength of the femtosecond laser from 760 to $800 \mathrm{~nm}$. To exclude effects related to different absolute luminescence intensities, the power has been adjusted at each wavelength to keep the luminescence intensity from the virgin substrate constant at the same value that it takes for $790 \mathrm{~nm}$ at $170 \mathrm{~mW}$. In this way, no significant change of the domain contrasts for different excitation wavelengths could be found, as shown in Figure 13 b. Choosing equal excitation powers, however, yielded a decrease of the domain contrast with decreasing wavelength, because 
the luminescence intensity itself (and therefore also the excitation rate) increased when lowering the exciting wavelength. This latter effect may be due to the closeness of the three-photon excitation energy to the LNO bandgap energy.

An important consequence is that the domain contrasts presented in Table 4 (that have been measured at $170 \mathrm{~mW}$ and $790 \mathrm{~nm}$ ) need exactly the used power and excitation wavelength-and therefore the same excitation rate- to be reproduced. Using different objectives or femtosecond light sources may also yield deviations of the contrast values, as the local excitation rate depends also on the pulse shape and on the focus profile. The measurements in the next section were carried out again at $790 \mathrm{~nm}$ and $170 \mathrm{~mW}$ as well.

\subsection{Thermal Decay of the Domain Contrast}

To obtain more insights into the physics of the microscopic origins of the luminescence domain contrast, we investigated its behavior under annealing the Mg:CLN samples at higher temperatures up to $200{ }^{\circ} \mathrm{C}$. We point out again that in this temperature range no chemical reactions with the surrounding air affect the crystal structure and composition. These annealing experiments confirmed that the luminescence domain contrast is also related with defect dipoles that contain lithium vacancies which can thermally rearrange after poling. In the first kind of annealing series we wanted to record the behavior of the domain contrast under stepwise rising temperatures. The samples have been put repeatedly into an oven for each $15 \mathrm{~min}$ at a constant annealing temperature, and in each new step the temperature has been chosen by $15 \mathrm{~K}$ higher than in the preceding step; thus the range from 60 to $200^{\circ} \mathrm{C}$ has been covered. After each annealing step the domain contrasts have been measured on at least three different positions on the samples. The results for $5 \mathrm{~mol} \% \mathrm{Mg}$ :CLN and $2.5 \mathrm{~mol} \% \mathrm{Mg}: \mathrm{SLN}$ are shown in Figure 14a. While the domain contrasts remain constant for temperatures up to $80^{\circ} \mathrm{C}$ they drop to a small fraction of their initial values within the range from 100 to $160^{\circ} \mathrm{C}$. For $5 \mathrm{~mol} \% \mathrm{Mg}$ :CLN it drops to around 1\%, for $2.5 \mathrm{~mol} \% \mathrm{Mg}$ :SLN it drops to around 1.5\%. For higher temperatures up to $200{ }^{\circ} \mathrm{C}$ these residual domain contrasts remain constant. This behavior corresponds well with the annealing-induced reduction of the internal fields observed by Gopalan et al., as shown in Figure 10, where the internal field changes drastically around around $150^{\circ} \mathrm{C}$ [26]. Although the internal field in undoped CLN and the domain contrasts in Mg:CLN are based on different kinds of defect complexes, these analogies underline the rearrangement of lithium vacancies as a common cause for the thermal realignment.

A further annealing series carried out at a constant temperature showed an exponential decay of the domain contrast over the time to the saturation value $C^{\infty}$, as shown in Figure $14 \mathrm{~b}$ for $5 \mathrm{~mol} \%$ $\mathrm{Mg}: \mathrm{CLN}$ at $110{ }^{\circ} \mathrm{C}$. However, while the domain contrast dropped in the first series to $1 \%$ at $200{ }^{\circ} \mathrm{C}$, $C^{\infty}$ lies between 1.5 and $2 \%$ in the $110{ }^{\circ} \mathrm{C}$ series. This shows that $C^{\infty}$ seems to be a function $C^{\infty}(T)$ of temperature. Figure 14c shows the measured values of $C^{\infty}(T)$ for $5 \mathrm{~mol} \% \mathrm{Mg}$ :CLN that have been extracted from annealing series of multiple samples for the Arrhenius plots. For the temperatures above $130^{\circ} \mathrm{C}$ one of the samples used at $130^{\circ} \mathrm{C}$ has been annealed at each temperature for $30 \mathrm{~min}$. This is long enough compared to the decay times at these temperatures to guarantee that the domain contrast approaches its saturation value close enough.

The exponential decay of the domain contrast over time is of a special physical interest. It indicates that the domain contrast is connected to the thermal crossing of a potential barrier $E_{\mathrm{a}}$ according to an Arrhenius-like process:

$$
C(t, T)=C^{\infty}(T)+A \exp [-t / \tau(T)]
$$

with

$$
\tau(T)=\tau_{0} \exp \left[E_{\mathrm{a}} / k T\right]
$$

and the activation energy $E_{\mathrm{a}}$ that can be easily determined by a linear fit of $\ln (\tau)$ over $1 / T$, using the $\tau$ values from the annealing series at different temperatures. 
a)
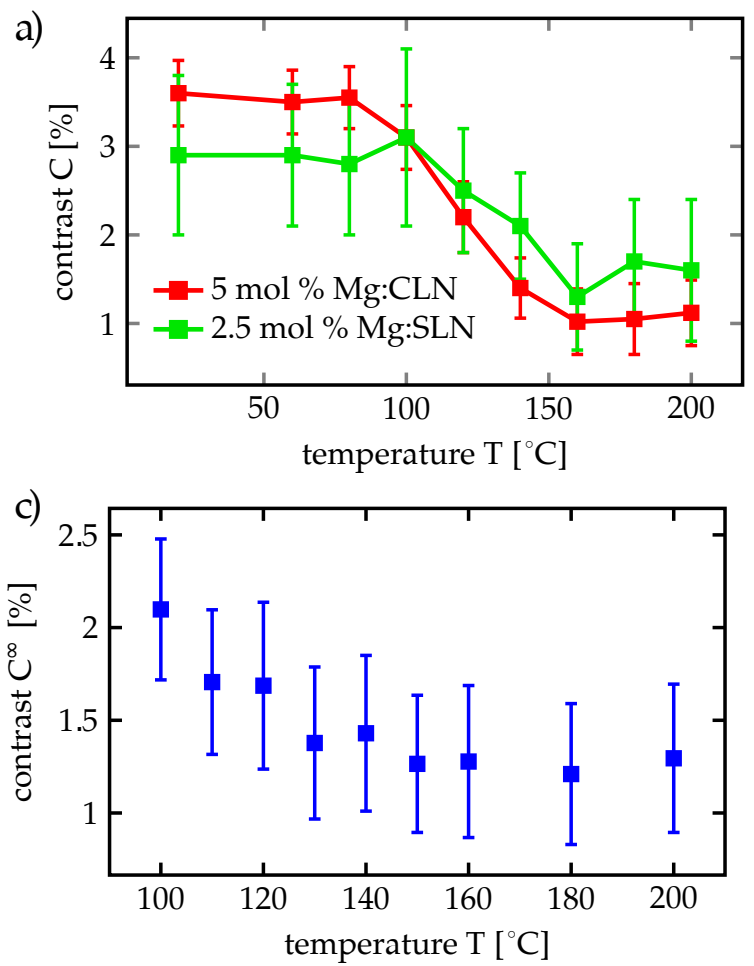

b)
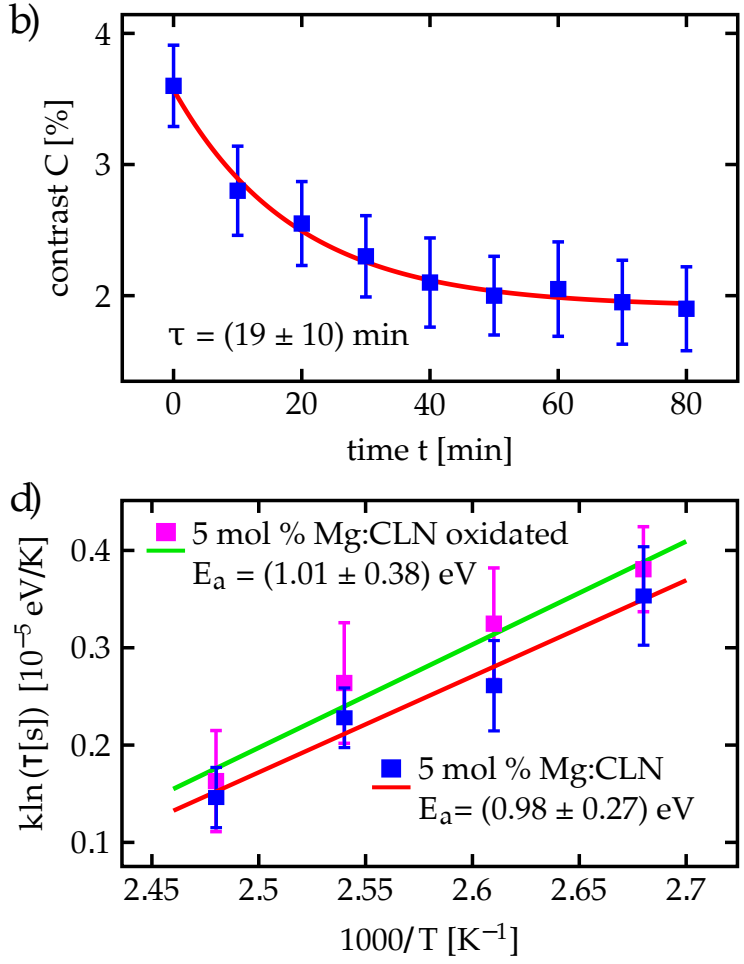

Figure 14. Annealing series and Arrhenius plots from domain contrast measurements with three-photon femtosecond excitation. (a) Measurements of $5 \mathrm{~mol} \% \mathrm{Mg}: \mathrm{CLN}$ and $2.5 \mathrm{~mol} \% \mathrm{Mg}: S L N$ with annealing steps of $15 \mathrm{~min}$ duration and increased temperatures for each step. (b) Exponential decay of the domain contrast in $5 \mathrm{~mol} \% \mathrm{Mg}: \mathrm{CLN}$ at an annealing temperature of $110^{\circ} \mathrm{C}$. (c) Saturation contrasts $C^{\infty}(T)$ for $5 \mathrm{~mol} \% \mathrm{Mg}$ :CLN at different annealing temperatures. The values up to $130^{\circ} \mathrm{C}$ are extracted from annealing series; for the temperatures above $130^{\circ} \mathrm{C}$ the sample used for the $130^{\circ} \mathrm{C}$ series has been used and annealed $30 \mathrm{~min}$ at each temperature. (d) Arrhenius plots for $5 \mathrm{~mol} \% \mathrm{Mg}: \mathrm{CLN}$ and for oxidated $5 \mathrm{~mol} \% \mathrm{Mg}$ :CLN and their respective fit curves and activation energies. The $5 \mathrm{~mol} \% \mathrm{Mg}$ :CLN curve in (a) and the curve (b) are reproduced with permission from P. Reichenbach et al., J. Appl. Phys. 2014, 115, 213509; the saturation contrast curve (c) and the Arrhenius plot for $5 \mathrm{~mol} \% \mathrm{Mg}$ :CLN in (d) from P. Reichenbach et al., Appl. Phys. Lett. 2014, 105, 122906 [18]; both with copyright (C) 2014 by AIP Publishing LLC.

Performing annealing series at $100,110,120$ and $130{ }^{\circ} \mathrm{C}$ for poled $5 \% \mathrm{Mg}: \mathrm{CLN}$ samples resulted in four values for the decay time $\tau(\mathrm{T})$. These values have been used to determine the activation energy $E_{\mathrm{a}}$ of the potential barrier in an Arrhenius plot of $\ln [\tau(\mathrm{T})]$ over $1 / \mathrm{T}$ as shown in Figure $14 \mathrm{~d}$. The resulting activation energy was $(0.98 \pm 0.27) \mathrm{eV}$. The existence of such an activation energy confirms that the thermally induced realignment of polar defect complexes involves indeed potential barrier crossings that lead to an Arrhenius-like process.

For the physical nature of the saturation contrast function $C^{\infty}(T)$, however, we did not find a conclusive model. A possible hypothesis would be a second Arrhenius-like process with an activation energy not too far away from the first one-as $C^{\infty}(T)$ changes also its value in the range of $150{ }^{\circ} \mathrm{C}$-but at a much smaller $\tau_{0}$. Such a smaller $\tau_{0}$ implies a much slower process that was not visible in the $100^{\circ} \mathrm{C}$ annealing series within our measurement time. Without a deeper understanding of the underlying mechanisms, however, these considerations are yet still speculative. Moreover, the measuered variations of $C^{\infty}(T)$ are too small compared to the statistical errors to allow a deeper quantitative investigation.

A comparison of the obtained activation energies with other known activation energies in LNO as listed in Table 6 allows conclusions about known processes being involved in the contrast decay and 
the underlying reconfiguration of the defect complexes. The contrast decay cannot be related with the mobility of bound electron polarons, hole polarons or bipolarons. As we already outlined in the second part, we obtain activation energies below $0.5 \mathrm{eV}$, according to the optical absorption energies of bound and hole polarons in LNO $[2,3,5]$ and the subsequent estimation of the activation energy of mobility as $1 / 4$ of the optical absorption energies [4]. The measured activation energy of mobility of the free polarons in LNO is below $0.2 \mathrm{eV}$ for a Mg doping of $5 \mathrm{~mol} \%$ and larger [16,99], while the bipolaron dissociation energy is by $0.27 \mathrm{eV}[4,53]$. On the other hand, the activation energies of heavier ions such as $\mathrm{Mg}^{2+}$ with $2.9 \mathrm{eV}[100]$ or $\mathrm{Nb}^{5+}$ are considerably higher than $1 \mathrm{eV}$.

Table 6. Reported activation energies $E_{\mathrm{a}}$ for the mobility of the occurring polarons and ions in LNO and for the domain contrast decay for different dopings (including $8 \mathrm{~mol} \% \mathrm{Mg}: \mathrm{CLT}$ and oxidated $5 \mathrm{~mol} \% \mathrm{Mg}: \mathrm{CLN})$. In the case of bipolaron the activation energy of dissociation is given. Except for the activation energy of the free hole $\mathrm{O}^{-}$(estimated to $1 / 4$ of the greatest estimated free hole polaron energy of $1.7 \mathrm{eV}$ ) and of oxidated $\mathrm{Mg}: \mathrm{CLN}$, these data are reproduced with permission from P. Reichenbach et al., Appl. Phys. Lett. 2014, 105, 122906 [18], copyright (C 2014 by AIP Publishing LLC.

\begin{tabular}{cccc}
\hline Phenomenon & Defect/Ion/Material & $\boldsymbol{E}_{\boldsymbol{a}}[\mathrm{eV}]$ & Ref. \\
\hline \multirow{4}{*}{ polaron } & $\mathrm{Nb}_{\mathrm{Nb}}^{4+}$ & $<0.2 \mathrm{eV}$ & {$[16,99]$} \\
& $\mathrm{Nb}_{\mathrm{Li}}^{4+}$ & $<0.4 \mathrm{eV}$ & {$[2,4]$} \\
& $\mathrm{Nb}_{\mathrm{Nb}}^{4+}+\mathrm{Nb}_{\mathrm{Li}}^{4+}$ & 0.27 & {$[4,53]$} \\
& $\mathrm{O}^{-}+\mathrm{V}_{\mathrm{Li}}$ & $<0.6 \mathrm{eV}$ & {$[2,3,5]$} \\
$\mathrm{O}^{-}$ & $<0.42 \mathrm{eV}$ & \\
\hline \multirow{3}{*}{ ion } & $\mathrm{Li}^{+}$ & $1.1-1.2 \mathrm{eV}$ & {$[103-106]$} \\
& $\mathrm{H}^{+}$ & $1.1 \mathrm{eV}$ & {$[101,102]$} \\
& $\mathrm{Mg}^{2+}$ & $2.9 \mathrm{eV}$ & {$[100]$} \\
\hline \multirow{4}{*}{ measured contrast decay } & $5 \% \mathrm{Mg}: \mathrm{CLN}$ & $(0.98 \pm 0.27) \mathrm{eV}$ & {$[18]$} \\
& $7 \%$ Mg:CLN (ox.) & $(1.06 \pm 0.33) \mathrm{eV}$ & \\
& $8 \% \mathrm{Mg}:$ CLT & $(1.01 \pm 0.38) \mathrm{eV}$ & {$[18]$} \\
& & $(0.82 \pm 0.28) \mathrm{eV}$ & {$[18]$} \\
\hline
\end{tabular}

However, in LNO an activation energy of about $1 \mathrm{eV}$ has been found for the mobility of $\mathrm{H}^{+}$ ions [101,102] and $\mathrm{Li}^{+}$ions [103-106]. Regarding these activation energies alone, the realignment of the defects leading to the contrast decay may be related with the mobility of $\mathrm{H}^{+}$or $\mathrm{Li}^{+}$ions. Due to the replacement of $\mathrm{Nb}_{\mathrm{Li}}$ by $\mathrm{Mg}_{\mathrm{Li}}$, the $\mathrm{Mg}_{\mathrm{Li}}+\mathrm{V}_{\mathrm{Li}}$ complex can be assumed to be the most prevalent kind of defect complexes in highly Mg-doped CLN. Probably they are also accompanied by defect dipoles containing $\mathrm{Mg}_{\mathrm{V}}$ and $\mathrm{V}_{\mathrm{Li}}$. For that reason the physical reorientation of these dipoles means indeed a rearrangement of the lithium vacancies that takes place by the hopping of lithium ions.

At this point, however, we wanted to definitively exclude that the mobility of $\mathrm{H}^{+}$ions and their mobility in LNO contributes also significantly to the domain contrast and its decay. To do so, we annealed samples of $5 \mathrm{~mol} \% \mathrm{Mg}: \mathrm{CLN}$ for $4 \mathrm{~h}$ at $1000{ }^{\circ} \mathrm{C}$ in a pure oxygen atmosphere in order to remove the $\mathrm{H}^{+}$ions. Absorption measurements showed a complete vanishing of the $\mathrm{OH}$ absorption band at $2.87 \mu \mathrm{m}$. After poling of domains in these samples, we observed the same domain contrasts as without oxidation. Therefore we concluded that hydrogen is not significantly involved in the material properties and processes responsible for the domain contrast and its thermal decay [18]. For this review article a further annealing series has been performed on such oxidated CLN samples, yielding the activation energy of $(1.06 \pm 0.33) \mathrm{eV}$ as shown also in Figure $14 \mathrm{~d}$, thus finally ruling out contributions from $\mathrm{H}^{+}$ions.

In this way, the central roles of lithium vacancies for the domain contrast, and of lithium ion mobility for its thermally induced decay, have been proven. On the one hand, the role of lithium confirms the importance of the $\mathrm{Mg}_{\mathrm{Li}}+\mathrm{V}_{\mathrm{Li}}$ defect complexes that realign thermally by mobility of lithium. Defect dipoles consisting of $\mathrm{V}_{\mathrm{Li}}$ and $\mathrm{Mg}_{\mathrm{V}}$ interstitials may contribute probably as well, due to 
a comparable domain contrast mechanism and the same kind of thermal rearrangement of the lithium vacancies. On the other hand, however, the domain contrast remaining at $200{ }^{\circ} \mathrm{C}$ indicates that further defect complexes like $\mathrm{Mg}_{\mathrm{Nb}}+3 \mathrm{Mg}_{\mathrm{Li}}$ contribute to the imperfect poling and the domain contrasts as well. This is similar to the incomplete realignment of the internal field in undoped CLN, probably due to $5 \mathrm{Nb}_{\mathrm{Li}}+4 \mathrm{~V}_{\mathrm{Nb}}$ complexes. Defect complexes based solely on $\mathrm{Mg}$ or $\mathrm{Nb}$ ions can expected to show considerably higher activation energies for reorientation, because larger ions would have to move through the crystal. As the number of $\mathrm{Mg}_{\mathrm{Nb}}+3 \mathrm{Mg}_{\mathrm{Li}}$ defect clusters increases with dopings above the doping threshold, we can expect also an increase of the saturation contrasts $C^{\infty}$ at higher dopings. Therefore future investigations of the domain contrasts and their saturation contrasts in dependence of the doping concentration would be interesting. Moreover, an incomplete restoration of internal fields and domain contrasts after a second inversion indicates the degradation of the crystal structure through repeated inversion. This behavior is more complex than the picture of a reversible inversion of the crystal structure around the fixed defect dipoles.

\section{Visible Luminescence and Its Contrasts in Lithium Tantalate}

While we intensively investigated the polaronic photoluminescence and its domain contrasts in LNO [17-19] we extended our investigations also to lithium tantalate (LTO), the ferroelectric material most similar to LNO [18]. As there is very scarce data on polarons and their energy levels in that material, our photoluminescence measurements on LTO are not as conclusive as for LNO. The measurements of the domain contrasts, however, showed much similarities with the domain contrasts in LNO, especially an activation energy of the contrast decay that is also in the range of $1 \mathrm{eV}$. On the one hand, new interesting features different from LNO may stimulate further investigations of polaron-related phenomena in LTO. On the other hand the phenomena comparable to LNO are a valuable complement to our work on lithium niobate.

\subsection{Luminescence Spectra of CLT}

Figure 15a shows the photoluminescence from congruent lithium tantalate (CLT), undoped as well as doped with $7 \mathrm{~mol} \%$ and $8 \mathrm{~mol} \% \mathrm{Mg}$, respectively [18]. The corresponding peak positions, widths and intensities under femtosecond excitation at $790 \mathrm{~nm}$ and $170 \mathrm{~mW}$, as estimated from Gaussian fits, are presented in Table 7. The samples show a similar kind of photoluminescence like LNO and the second-harmonic peak around $3.1 \mathrm{eV}$ as well. However, compared to the peak position of LNO luminescence, the luminescence peak from the doped samples is shifted by about $0.7 \mathrm{eV}$ to a value around $3.2 \mathrm{eV}$. Due to the limitations of the spectral transmission of our detection optics, the spectrum is cut off around $3.3 \mathrm{eV}$. This cutoff makes the Gaussian fits less accurate; thus the widths, maximum energies and areas of the peaks (the latter being used as estimations for the luminescence intensities) cannot be determined without larger errors [18]. Despite the uncertainties of the bandgap determination, comparative measurements indicated the LTO bandgap to be by $0.7 \mathrm{eV}$ larger than the LNO bandgap [40]. Assuming similar polaron energies like in LNO, these higher peak energies fit well with this shift of the measured bandgap energy.

The strong increase of the intensity from $7 \mathrm{~mol} \%$ to $8 \mathrm{~mol} \%$ indicates the doping threshold of $\mathrm{Mg}: \mathrm{CLT}$ to be at least in the range of $8 \mathrm{~mol} \%$. The higher doping threshold accords with the fact that the $\mathrm{Li} /(\mathrm{Li}+\mathrm{Nb})$ ratio of CLT deviates more from $50 \%(47.7 \%)$ [107] than in CLN $(48.45 \%)$ [1], resulting in a higher number of $\mathrm{Nb}_{\mathrm{Li}}$ anti-sites and lithium vacancies $\mathrm{V}_{\mathrm{Li}}$ that have to be replaced to reach the doping threshold. However, the most interesting observation is the spectrum of undoped CLT. In contrast to LNO, the luminescence of the undoped substrate is by only one order of magnitude weaker and the peak position is not at 3.2 but between 2.4 and $2.5 \mathrm{eV}$. The latter indicates the existence of a second luminescence center which does not occur in LNO. 

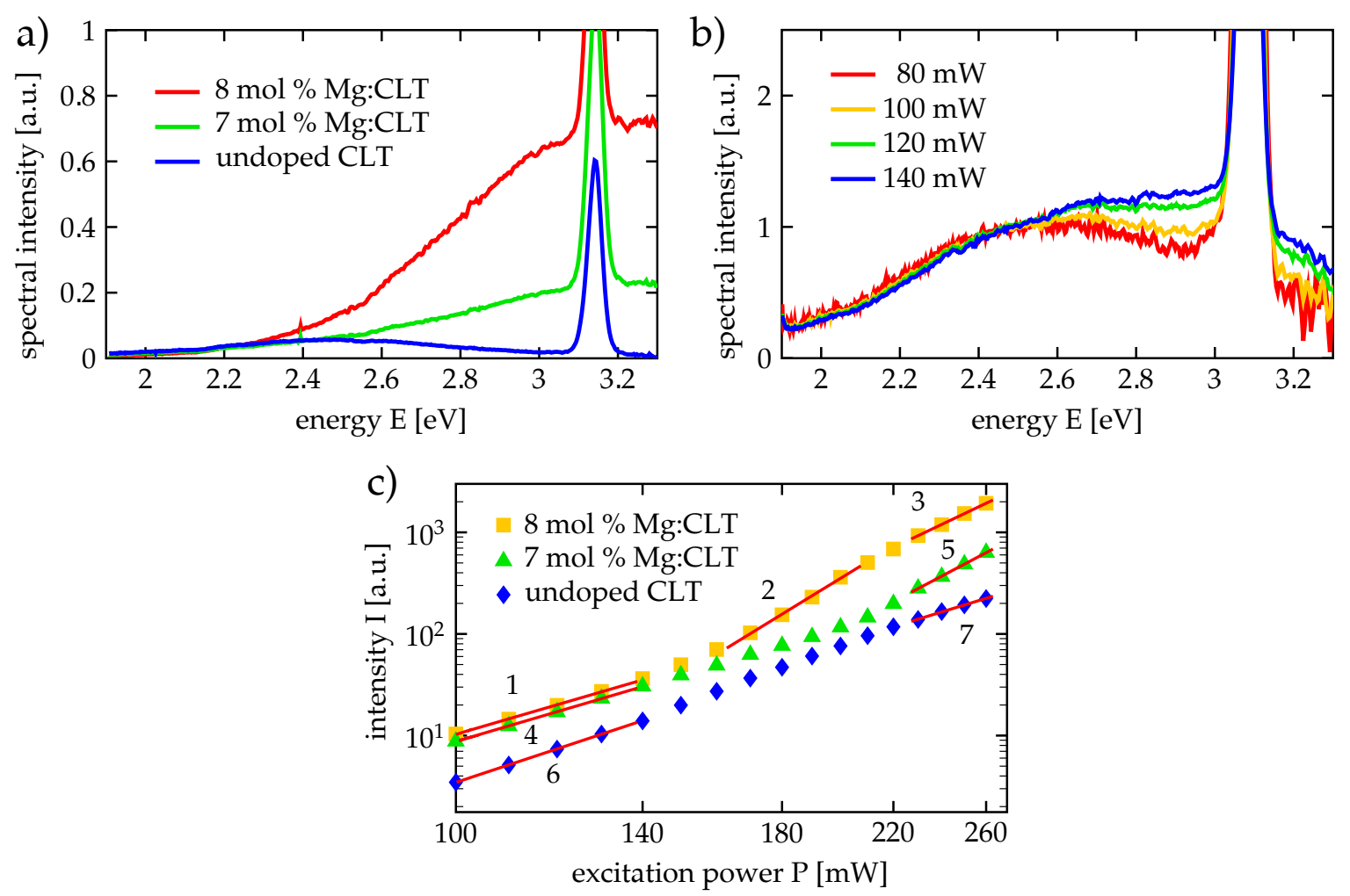

Figure 15. (a) Photoluminescence spectra and SHG peaks (at $3.1 \mathrm{eV}$ ) of $8 \mathrm{~mol} \% \mathrm{Mg}:$ CLT, $7 \mathrm{~mol}$ $\% \mathrm{Mg}:$ CLT and undoped CLT under three-photon femtosecond excitation at $790 \mathrm{~nm}$ and $170 \mathrm{~mW}$. To make the spectra comparable, the units are normalized in the same way as the diagrams in Figure 4. (b) Photoluminescence spectra of $8 \mathrm{~mol} \% \mathrm{Mg}$ :CLT for different smaller excitation powers with each curve normalized on its value at $2.5 \mathrm{eV}$. (c) Power dependence of photoluminescence intensity for undoped CLT and the 7 and $8 \mathrm{~mol} \% \mathrm{Mg}$ dopings in double-logarithmic plots. Selected parts of the curves are fitted by straight lines that correspond to fitted power functions $P^{n}$. These fits are labeled by numbers. The slopes of the fitting lines in the double-logarithmic plot correspond to the exponents $n$ of the fitted power functions $P^{n}$ which are presented in Table 8. (a) is reproduced with permission from P. Reichenbach et al., Appl. Phys. Lett. 2014, 105, 122906 [18], copyright (c) 2014 by AIP Publishing LLC.

Table 7. Peak positions $E$, widths $w$ and luminescence intensities $I / I_{5} \mathrm{~mol} \% \mathrm{Mg}: \mathrm{CLN}$ from Mg-doped and undoped CLT. The intensities have been estimated from the areas of Gaussian fits of the measured luminescence spectra, and have been normalized to the area of the $5 \mathrm{~mol} \% \mathrm{Mg}$ :CLN spectrum as the reference intensity. These data are reproduced with permission from P. Reichenbach et al., Appl. Phys. Lett. 2014, 105, 122906 [18], copyright (C) 2014 by AIP Publishing LLC.

\begin{tabular}{cccc}
\hline Doping & $\boldsymbol{E}[\mathrm{eV}]$ & $\boldsymbol{w}[\mathrm{eV}]$ & $\boldsymbol{I}_{\mathbf{5}} \mathbf{5 ~ m o l} \% \mathrm{Mg}: \mathrm{CLN}$ [a.u.] \\
\hline $8 \mathrm{~mol} \%$ & $3.2 \pm 0.2$ & $0.40 \pm 0.10$ & $0.98 \pm 0.05$ \\
$7 \mathrm{~mol} \%$ & $3.3 \pm 0.2$ & $0.45 \pm 0.10$ & $0.34 \pm 0.10$ \\
none & $2.5 \pm 0.2$ & $0.25 \pm 0.05$ & $0.04 \pm 0.01$ \\
\hline
\end{tabular}

While the power dependence of the visible photoluminescence in LNO revealed a third-order process [17], the power dependence of the visible luminescence in LTO shows a much more complex behavior, as shown in Figure 15b,c. While no significant change of the shape of the spectrum occurred for CLN and SLN, the spectrum of doped CLT changes its shape at small powers. Figure 15b shows normalized luminescence spectra from $8 \mathrm{~mol} \% \mathrm{Mg}$ :CLT for excitation powers from 80 to $140 \mathrm{~mW}$. For the smaller powers the luminescence peak about $3 \mathrm{eV}$ disappears and the spectral maximum turns then out to be located around $2.6 \mathrm{eV}$. For even smaller powers the spectrum may approach the 
luminescence spectrum of undoped CLT with its maximum close to $2.5 \mathrm{eV}$. This indicates that the luminescence center being excited in undoped CLT is also excited in doped CLT, being dominant at low excitation powers.

Figure $15 \mathrm{c}$ shows the dependence of the luminescence intensity on the excitation power for undoped CLT as well as 7 and $8 \mathrm{~mol} \% \mathrm{Mg}$ :CLT, respectively. For different excitation intensities $P$ parts of the luminescence intensity can be fitted with power functions $P^{n}$. In the double-logarithmic plot in Figure $15 \mathrm{c}$ the exponents $n$ are indicated by the slopes of the linear fits. The resulting exponents are presented in Table 8; the fits are labeled in Figure 15c. While the investigated Mg:CLN showed exponents around 3, CLT shows always exponents larger than 3 . The most interesting phenomenon, however, are the pronounced variations of the slope around 140 and $220 \mathrm{~mW}$ for $8 \mathrm{~mol} \% \mathrm{Mg}$ :CLT. While the exponent is around 3.7 at the low-power regime and around 6.0 at the high-power regime of the curve, it reaches a maximum of of about 7.7 in the range between 140 and $220 \mathrm{~mW}$. Furthermore, the lower luminescence from $7 \mathrm{~mol} \% \mathrm{Mg}$ :CLT shows a constant exponent of about 4 up to $220 \mathrm{~mW}$ and the inset of a larger slope at higher powers. For the undoped CLT no such kink appears; the exponent is rather constant around 4 .

Table 8. Exponents $n$ from the numbered fit lines in Figure $15 \mathrm{c}$ with the $\mathrm{Mg}$ dopings and power ranges of the fitted curve segments.

\begin{tabular}{cccc}
\hline Fit No. & $\boldsymbol{n}$ & $\begin{array}{c}\text { Mg Doping } \\
\text { [mol \%] }\end{array}$ & $\begin{array}{c}\text { Power Range } \\
{[\mathbf{m W}]}\end{array}$ \\
\hline 1 & $3.7 \pm 0.1$ & 8 & $100-140$ \\
2 & $7.7 \pm 0.3$ & 8 & $170-210$ \\
3 & $6.0 \pm 0.1$ & 8 & $230-260$ \\
4 & $3.7 \pm 0.1$ & 7 & $100-140$ \\
4 & $6.6 \pm 0.1$ & 7 & $230-260$ \\
4 & $4.2 \pm 0.1$ & 0 & $100-140$ \\
4 & $3.8 \pm 0.1$ & 0 & $230-260$ \\
\hline
\end{tabular}

The presence of the slope variations in the power curves of Mg-doped CLT may be interpreted as follows: The exponents in the range of 3.7 for the lower excitation powers correspond to spectra with the peak at $2.5 \mathrm{eV}$ dominating, which is also the case for undoped CLT in the full range of the investigated excitation power. For higher powers the $3.2 \mathrm{eV}$ peak appears in doped CLT and begins to dominate due to its faster increase. This strong nonlinearity leads to the slope variations of the luminescence intensity curve. The reduction of the exponent for $8 \mathrm{~mol} \% \mathrm{Mg}$ :CLT at the highest displayed excitation powers-above the second kink-may indicate some kind of saturation as the $3.2 \mathrm{eV}$ peak grows slower. For $7 \mathrm{~mol} \% \mathrm{Mg}$ :CLT, however, the inset of a higher slope has shifted to higher powers and begins around $220 \mathrm{~mW}$. This shift to higher powers is probably related to the relative weakness of the overall luminescence intensity in $7 \mathrm{~mol} \% \mathrm{Mg}$ :CLT compared to $8 \mathrm{~mol} \%$ $\mathrm{Mg}: \mathrm{CLT}$. As the slope variations depend on the luminescence intensity, obviously larger excitation intensities are required to reach this regime of varying slopes.

For CLT we see clearly the presence of two luminescence centers instead of a single luminescence center from free polaron and hole polaron recombination. The power-dependent measurements indicate that a saturation of the $2.5 \mathrm{eV}$ band may occur at higher powers, leading to an increasing recombination of excited charge carriers over the other luminescence center around $3.2 \mathrm{eV}$. We have also checked the spectra with the nanosecond laser setup, although we did not carry out thorough time-dependent measurements as in Section 2.5. However, in the pulsed UV laser setup only the $2.5 \mathrm{eV}$ peak was visible and not the $3.2 \mathrm{eV}$ peak. This difference to the curves recorded with the fs laser setup may be caused by the lower local excitation densities of the unfocused UV beam compared to the focused femtosecond beam, leading to a vanishing of the $3.2 \mathrm{eV}$ peak similar as in Figure 15b.

To a full elucidation of these processes not only thorough time-dependent measurements but also a determination of the polaron energies by reduction, absorption and pump-probe measurements on 
reduced and doped LTO samples would be necessary. This would allow to assign each luminescence band to the involved polaron states. One of the few known facts on polaron states in LTO is the absence of a free polaron peak in the range of $1 \mathrm{eV}$, as found in a measurement during generating electron and hole polarons by femtosecond-pulsed excitation at $400 \mathrm{~nm}$ [108]. This result has been explained by the presence of defects trapping all free polarons. However, a luminescent recombination of (mobile) free hole polarons with bound electron polarons would still be possible. The two appearing luminescence centers may then arise from two different species of bound electron polarons, trapped at two different kinds of defects, respectively. As the $3.2 \mathrm{eV}$ peak does not appear in undoped CLT, it is therefore probably related to $\mathrm{Mg}$-based defects. However, without a deeper knowledge on the polaron states in LTO this consideration is still speculative.

These results underline the differences between polaron states in LNO and LTO and suggest further investigations on polarons in LTO.

\subsection{Luminescence Domain Contrasts in CLT}

For the visible luminescence in CLT the same kind of domain contrasts appears as for LNO. The measured domain contrasts are shown in Table 9 . With values up to $30 \%$ they are considerably higher than those of LNO. Analogous to Mg:CLN we can assign these domain contrasts to the presence of $\mathrm{Mg}_{\mathrm{Li}}+\mathrm{V}_{\mathrm{Li}}$ as well as $\mathrm{Mg}_{\mathrm{V}}$-based defect complexes. However, with dopings of 7-8 mol \% the density of these defect complexes is not much higher than in the Mg-doped CLN samples, which rules out the different doping levels as an important reason for the high domain contrasts. This indicates that the defect complexes have probably a stronger influence on the nonradiative recombination than in LTO.

Figure 16a shows a luminescence scan image from the triangular domains in $7 \mathrm{~mol} \% \mathrm{Mg}$ :CLT. Figure 16b shows an inverted area of $8 \mathrm{~mol} \% \mathrm{Mg}$ :CLT. In the latter doping, no complete inversion is possible, as irregular, few $\mu \mathrm{m}$ sized domains of virgin polarization still remain in the substrate after switching of a large area. In this scan most of them show again the characteristic triangular shape.

a)
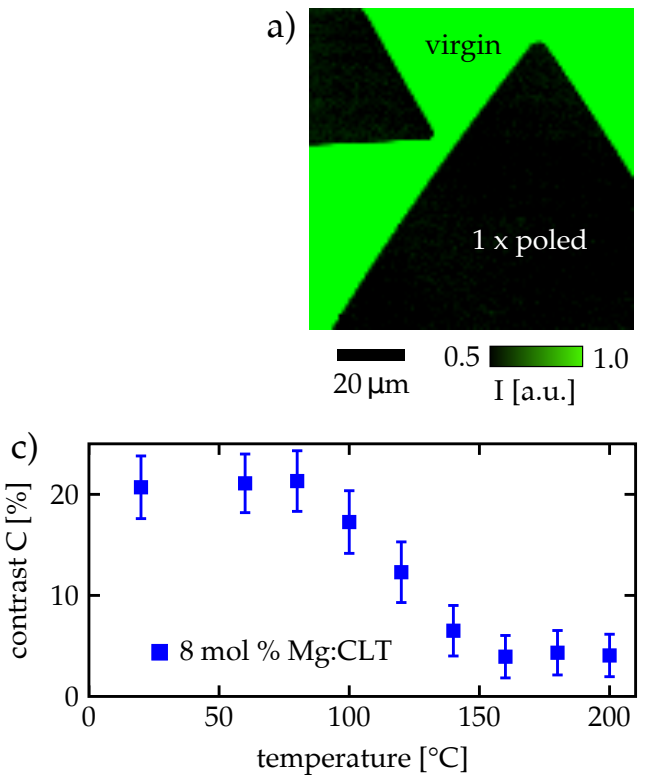
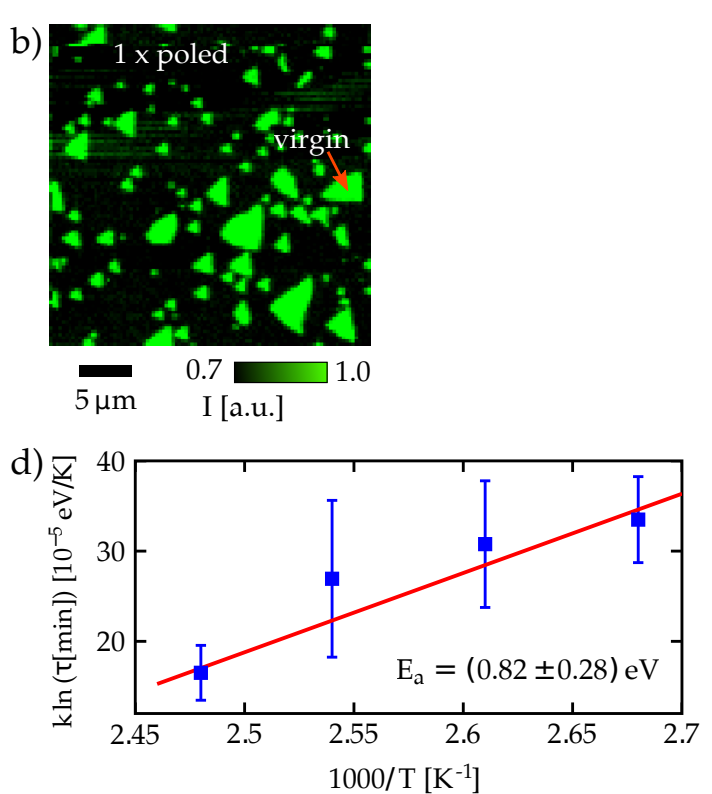

Figure 16. Photoluminescence images of domains and annealing series at Mg-doped CLT under three-photon femtosecond excitation. (a) Photoluminescence images of domains in $7 \mathrm{~mol} \% \mathrm{Mg}$ :CLT. (b) Non-inverted domains of virgin polarization in poled $8 \mathrm{~mol} \% \mathrm{Mg}$ :CLT. (c) Annealing series at stepwise rising temperatures for $8 \mathrm{~mol} \% \mathrm{Mg}$ :CLT with measurements of the domain contrast after annealing times of $15 \mathrm{~min}$ at each temperature. (d) Arrhenius plot from $100{ }^{\circ} \mathrm{C}$ to $130{ }^{\circ} \mathrm{C}$ for $8 \mathrm{~mol} \%$ Mg:CLT. (a,b,d) are reproduced with permission from P. Reichenbach et al., Appl. Phys. Lett. 2014, 105, 122906 [18], copyright (C) 2014 by AIP Publishing LLC. 
An annealing series, performed at stepwise rising temperatures with $8 \mathrm{~mol} \% \mathrm{Mg}$ :CLT, showed similar results as for LNO and is given in Figure 16c. The curve shows a drop from $20 \%$ to around $4 \%$ around $150{ }^{\circ} \mathrm{C}$. Note that Gopalan et al. found the drop of the internal field in the same temperature range for LTO as well [25]. The Arrhenius plot in Figure $16 \mathrm{~d}$ yields an activation energy of $(0.88 \pm 0.27) \mathrm{eV}$, i.e., again in the range of $1 \mathrm{eV}$. Despite scarce data on activation energies in CLT, this value indicates an involvement of the $\mathrm{Li}^{+}$mobility in the contrast decay and the major role of $\mathrm{Mg}_{\mathrm{Li}}+\mathrm{V}_{\mathrm{Li}}$ defect complexes in LTO as well. Moreover, the measurements discussed already in Section 3.3 showed also a pronounced power dependence of the domain contrast in $8 \mathrm{~mol} \% \mathrm{Mg}$ :CLT.

Table 9. Multiphoton luminescence domain contrasts for undoped and Mg-doped CLT [18]. These data are reproduced with permission from P. Reichenbach et al., Appl. Phys. Lett. 2014, 105, 122906 [18], copyright (c) 2014 by AIP Publishing LLC.

\begin{tabular}{cc}
\hline Mg Doping [mol \%] & Contrast [\%] \\
\hline 0 & $27.2 \pm 2.5$ \\
7 & $30.2 \pm 3.6$ \\
8 & $20.4 \pm 2.9$ \\
\hline
\end{tabular}

\subsection{Three-Dimensional Domain Pattern Visualization in Mg:CLT}

While most domain images show hexagonal (LNO) or triangular (LTO) domain shapes, we found many irregular residual domain structures in $8 \mathrm{~mol} \% \mathrm{Mg}$ :CLT. These irregular structures appear in each inverted $8 \mathrm{~mol} \% \mathrm{Mg}$ :CLT sample as certain intrinsic properties of that material impede a complete inversion. These domains deserve a closer look, as some of the smaller ones show also a triangular shape. The advantage of multiphoton photoluminescence microscopy to penetrate the substrate deeply makes it possible to investigate domain shapes within the bulk, as it is also feasible with Cerenkov SHG [23,109-111]. Figure 17 shows a series of scans of larger irregular domains in different depths up to $50 \mu \mathrm{m}$. It turns out that within the bulk most of the larger irregular domains shrink to small triangular domains within the first $30 \mu \mathrm{m}$ of the bulk. These triangles keep their shape in larger depths, as there is almost no change from 30 to $50 \mu \mathrm{m}$. Thus, it also turns out that only a small part of the $8 \mathrm{~mol} \% \mathrm{Mg}$ :CLT bulk volume remains actually in the virgin state. It also indicates that the minimization of domain wall energies causes triangular prism-shaped domains within the bulk-as well-known for the domain structures in the lower doped CLT. Usually, the domains keep their shape from surface to surface. This special behavior, however, is probably due to a strong domain wall pinning on the surface that impedes a complete switching on and close to the surface. By this effect large domains remain uninverted near the surface. Within the bulk, these domains continue to thin triangular pillars-thus probably minimizing the domain surface energy. Domain wall pinning has already been observed in LNO [112], however, in $8 \mathrm{~mol} \% \mathrm{Mg}$ :CLT the strong doping may show pronounced influences on the surface structure being responsible for that pinning.

A closer look to the scans shows that, by going e.g., $5 \mu \mathrm{m}$ deeper into the substrates, domain walls shrink over distances in the order of a few $\mu \mathrm{m}$. This indicates near-surface inclination angles in the order of some $10^{\circ}$, which is huge compared to the tiny inclination angles far below $1^{\circ}$ that have been measured in CLN [65,110]. Due to the relation between domain wall conductivity and inclination angles [65], in $8 \mathrm{~mol} \% \mathrm{Mg}$ :CLT large domain wall conductivities could be found-at least in the near-surface bulk where the shrinking to the small triangle domains takes place. 

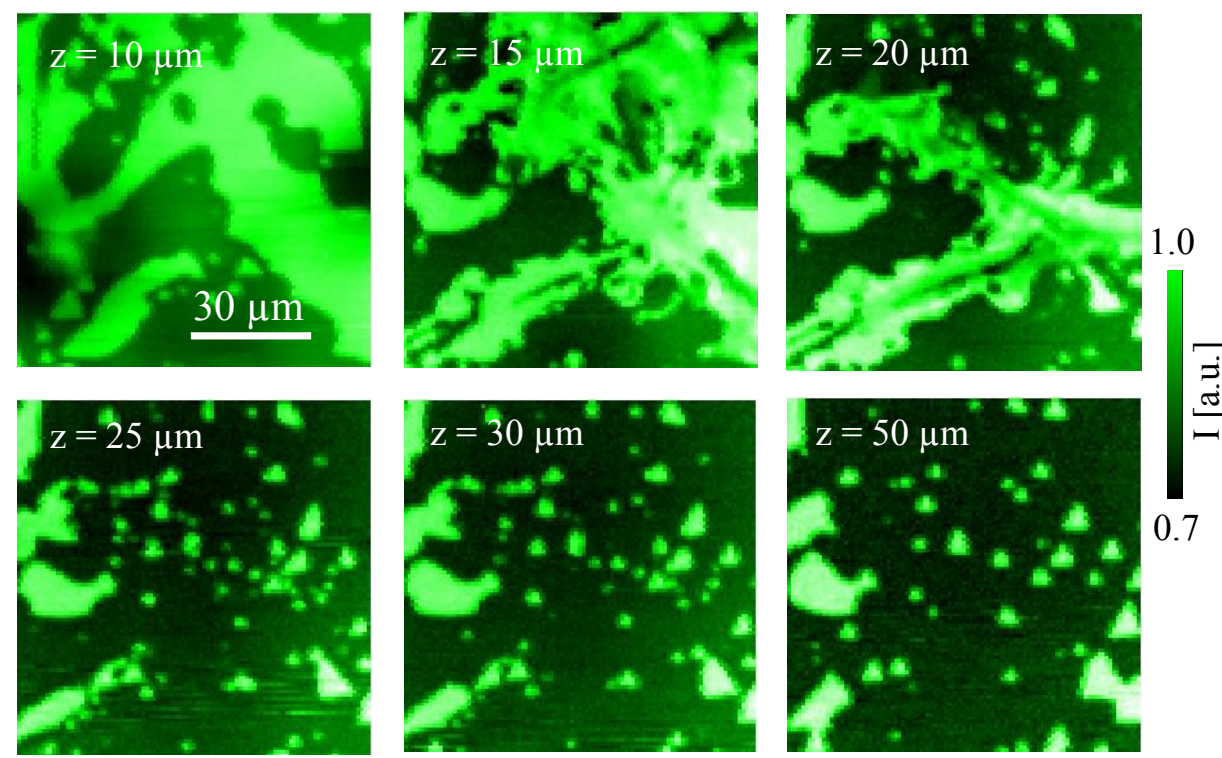

Figure 17. Photoluminescence contrast scans of the irregular domains in (partially) inverted $8 \mathrm{~mol} \%$ Mg:CLT at different penetration depths under three-photon femtosecond excitation.

\section{Summary and Outlook}

In this concluding section we give a summary of our review and point out open questions that deserve a deeper investigation.

Polarons in LNO and LTO are of great importance for absorption and photoluminescence phenomena in these materials. Several groups carried out absorption measurements in order to elucidate the energy levels and physical properties of the different polaron types $[2,3,5,16,53]$. Such measurements required these inter-band states to be filled with charge carriers, either by reduction or by optical pumping. These investigations confirmed that both electrons and holes appear either in free quasiparticle states (free electron and hole polarons) or in states bound to defect sites (bound electron and hole polarons). Furthermore, free electron polarons can join with bound electron polarons to form bipolarons. As electrons from $\mathrm{Fe}^{2+}$ defect ions can be excited into bound polaron states on neighboring $\mathrm{Nb}_{\mathrm{Li}}$ anti-sites, doping $\mathrm{LNO}$ with a higher $\mathrm{Fe}^{2+}$ concentration results in a strong enhancement of absorption as well as of photoluminescence from bound polarons.

Data on the transitions of hole polarons are only available from bound hole polarons; from free hole polarons even direct observations of their energy levels are lacking. The only known transition-the recombination with free electron polarons-would in principle allow to determine the energy level of the free hole polarons. The uncertainty of the bandgap, however, leaves still a range from 0.3 to $1.7 \mathrm{eV}$. For that reason an independent verification and determination of basic hole polaron parameters like energy and mobility is essential for a better understanding of the polaron recombination physics.

There are different kinds of photoluminescence effects mediated by polarons. The bound polaron luminescence at $1.5 \mathrm{eV}$ is caused by excitation of the bound polaron state to a shallowly trapped excited state on the lower edge of the bandgap and the subsequent relaxation back to the bound polaron ground state. It can be excited by strong $\mathrm{CW}$ or picosecond-pulsed visible laser light. As it takes place exclusively on the hosting $\mathrm{Nb}_{\mathrm{Li}}$ anti-sites, its decay is mono-exponential. Furthermore, this luminescence depends strongly on iron doping, as from $\mathrm{Fe}^{2+}$ ions electrons can be excited into bound polaron states.

In addition to the $1.5 \mathrm{eV}$ band, another luminescence band at $1.3 \mathrm{eV}$ has been reported, too. However, no investigations of that band have been continued in later publications, probably due to difficulties to differentiate both peaks in detection. The only facts known to us are that this peak is not very sensitive to stoichiometry and that it disappears upon reduction. It may be possible that the 
$1.3 \mathrm{eV}$ peak stems from an unknown radiative transition involving either free or bound hole polarons. The number of the hole polarons would indeed strongly decrease under reduction due to the increased presence of negative charge carriers. To properly judge on these possibilities, however, a deeper knowledge about the hole polaron states and their transitions is necessary.

The other important luminescence band in LNO is the visible luminescence band around $2.6 \mathrm{eV}$ from the recombination of free electron polarons with free hole polarons. It can be excited by UV illumination or by multiphoton excitation induced by near-infrared femtosecond pulses. The recombination of electron polarons with hole polarons, separated by randomly distributed distances, yields a stretched-exponential decay, resulting from the superposition of a multitude of different decay times. Like the $1.5 \mathrm{eV}$ peak, the visible luminescence is considerably influenced by doping, but in a quite different manner. It is strongly enhanced by doping with $\mathrm{Mg}$ and other optical damage resistant dopings around the doping threshold (in the range of $5 \mathrm{~mol} \%$ in CLN and $1 \mathrm{~mol} \%$ in SLN for Mg doping). The point is that such kind of doping reduces the number of $\mathrm{Nb}_{\mathrm{Li}}$ anti-sites. A similar dependence of the luminescence on stoichiometry confirms the importance of $\mathrm{Nb}_{\mathrm{Li}}$ for luminescence quenching. Obviously the presence of these defects is necessary for non-radiative recombination channels that quench the visible luminescence. A credible proposal by Tang et al. [86] suggests a non-radiative recombination channel via bipolaron formation that would indeed require $\mathrm{Nb}_{\mathrm{Li}}$ anti-sites. The temperature-dependent luminescence quenching in the range of the doping threshold, however, indicates at least one further concurring nonradiative process. The recombination of free polarons with the bound hole polarons trapped at the $\mathrm{Mg}_{\mathrm{Li}}+\mathrm{V}_{\mathrm{Li}}$ defect clusters seems to be the most credible possibility. In addition, it seems to be consistent with the activation energies of the lifetimes $\tau_{1}, \tau_{2}$ that agree roughly with electron and polaron hopping energies, respectively. In addition to the mentioned lithium-occupying defects $\mathrm{Nb}_{\mathrm{V}}$ and $\mathrm{Mg}_{\mathrm{V}}$ interstitials, located in regularly empty oxygen octahedra, may contribute in the same way to the nonradiative recombination. However, more thorough experimental and theoretical investigations of all occurring recombination processes are necessary to model accurately their relationship to all occurring defects as well as their concurrence behavior, thus resolving several remaining open questions. This deeper understanding of these processes requires also a comprehensive knowledge about the interplay of different influences like polaron hopping, electron-phonon interaction as well as a possible deep-temperature transition into an itinerant polaron band.

First measurements on the $2.6 \mathrm{eV}$ luminescence have been made by Koppitz, Klose et al. $[15,16]$ using UV illumination. Our luminescence measurements using three-photon photoexcitation and nanosecond pulsed UV illumination for time-dependent measurements confirmed their major findings [17] and resulted in a deeper knowledge about the luminescence domain contrasts [17,18] as well as the stretched-exponential time-dependent behavior [19].

Our investigations on luminescence by three-photon excitation included Mg-doped CLT, too. This material showed a luminescence spectrum in the visible wavelength range, however, with a peak energy around a larger value of $3.2 \mathrm{eV}$. At smaller excitation powers as well as for undoped LTO another luminescence band in the range of about $2.5 \mathrm{eV}$ appears. The appearance of two luminescence centers, the absence of the polaron $1 \mathrm{eV}$ absorption peak [108] as well as the strong nonlinearities in the power-dependence of the multiphoton luminescence (apparently related with the spectral shift of the luminescence peak) underline the necessity of a deeper investigation of the polaron states and optical processes in LTO.

Table 10 gives an overview about all used ferroelectric materials and the measurements performed on them in our group. Three-photon luminescence spectra have been taken from all samples, time-dependent luminescence curves from most of them. The remaining measurements dealt with the domain contrasts of the visible photoluminescence (taken from almost all samples) and annealing series (taken from selected samples). Thus, we have covered a broader range of dopings and stoichiometries in our measurements. 
Table 10. Materials and applied methods for our presented measurements. The CLN and CLT samples had been purchased from Crystal Technology and Yamaju Ceramics, while the SLN samples had been prepared in the Wigner Research Center for Physics in Budapest.

\begin{tabular}{|c|c|c|c|c|c|c|c|}
\hline Material & Dopant & Doping [mol \%] & $\begin{array}{l}\text { Multiphoton } \\
\text { Luminescence } \\
\text { Spectroscopy }\end{array}$ & $\begin{array}{l}\text { Time-Resolved } \\
\text { Luminescence } \\
\text { Spectroscopy }\end{array}$ & $\begin{array}{c}\text { Ferroelectric } \\
\text { Domain Contrast } \\
\text { Evaluation }\end{array}$ & $\begin{array}{c}\text { Thermal } \\
\text { Annealing } \\
\text { Series }\end{array}$ & $\begin{array}{l}\text { Arrhenius-Type } \\
\text { Activation Energy } \\
\text { Extraction }\end{array}$ \\
\hline \multirow{6}{*}{ CLN } & none & - & $x$ & & & & \\
\hline & \multirow{5}{*}{$\mathrm{Mg}$} & 1 & $x$ & & & & \\
\hline & & 2 & $x$ & & & & \\
\hline & & 3 & $\times$ & $x$ & & & \\
\hline & & 5 & $x$ & $x$ & $x$ & $x$ & $x$ \\
\hline & & 7 & $\times$ & $x$ & $x$ & $\times$ & $\times$ \\
\hline \multirow{9}{*}{ SLN } & none & - & $x$ & $x$ & & \multirow{9}{*}{$\times$} & \\
\hline & & 1 & $x$ & $x$ & $\times$ & & \\
\hline & $\mathrm{Mg}$ & 2.5 & $\times$ & $x$ & $x$ & & \\
\hline & & 4.5 & $x$ & $x$ & $x$ & & \\
\hline & $\mathrm{Zr}$ & 0.085 & $x$ & $\times$ & $x$ & & \\
\hline & $\mathrm{Sc}$ & 0.5 & $\times$ & $\times$ & & & \\
\hline & In & 0.5 & $x$ & $x$ & $x$ & & \\
\hline & $\mathrm{Hf}$ & 0.55 & $\times$ & $x$ & $\times$ & & \\
\hline & $\mathrm{Zn}$ & 0.7 & $\times$ & $\times$ & $x$ & & \\
\hline \multirow{3}{*}{ CLT } & none & - & $x$ & $x$ & $x$ & & \\
\hline & \multirow{2}{*}{$\mathrm{Mg}$} & 7 & $\times$ & $\times$ & $\times$ & & \\
\hline & & 8 & $\times$ & $x$ & $x$ & $x$ & $\times$ \\
\hline
\end{tabular}

The luminescence domain contrasts show that the visible polaron-mediated luminescence is sensitive to modifications of the crystal structure that result from imperfect electric poling. Due to dipolar defect complexes as $\mathrm{Nb}_{\mathrm{Li}}+4 \mathrm{~V}_{\mathrm{Li}}$ [29] or $\mathrm{Mg}_{\mathrm{Li}}+\mathrm{V}_{\mathrm{Li}}$ keeping their original orientation during the electric poling, the crystal structure does not completely invert. Instead of that these defect dipoles arrive in a frustrated state. These defect complexes are responsible for changes of the "internal field" [24,25] and also influence the luminescence from defect ions $[23,86,94,95]$ as well as the visible luminescence from polaron recombination [17-19]. Note that $\mathrm{Nb}_{\mathrm{Li}}$-based defect complexes are dominant in undoped $\mathrm{LNO}$, while $\mathrm{Mg}_{\mathrm{Li}}$-based defect complexes dominate at $\mathrm{Mg}$ dopings around the doping threshold.

Annealing the samples to about $150{ }^{\circ} \mathrm{C}$ yields a reduction of the luminescence domain contrasts to a small fraction of their initial values-according to the realignment of the internal field in the same temperature range. Both effects can be interpreted as a thermally activated reorientation of the frustrated dipolar defects. Annealing series for $5 \mathrm{~mol} \%$ and $7 \mathrm{~mol} \% \mathrm{Mg}: \mathrm{CLN}$, oxidated $5 \mathrm{~mol} \%$ $\mathrm{Mg}: \mathrm{CLN}$ and $8 \mathrm{~mol} \% \mathrm{Mg}$ :CLT show activation energies of about $1 \mathrm{eV}$, suggesting that the reorientation of defect dipoles is mediated by the thermal mobility of lithium. This is consistent with our assumption that the $\mathrm{Mg}_{\mathrm{Li}}+\mathrm{V}_{\mathrm{Li}}$ defect complex plays a dominant role for the domain contrasts, as its reorientation takes place by a rearrangement of the lithium vacancy by hopping of lithium ions. The same can be the case with $\mathrm{Mg}_{\mathrm{V}}$ interstitials accompanied by lithium vacancies as well. The remaining domain contrasts after annealing, however, indicate the contribution of other kinds of defect complexes to the domain contrast, most probably from the type $\mathrm{Mg}_{\mathrm{Nb}}+3 \mathrm{Mg} \mathrm{Li}_{\mathrm{Li}}$. However, this model of imperfect poling does not yet explain the incomplete restoration of the virgin state after a second inversion. This incompleteness is due to a degradation of the crystal structure through repeated inversion and results in reduced internal fields and luminescence domain contrasts, as the domain contrast of $2.5 \%$ measured at doubly inverted domains in a singly inverted substrate. Behind this degradation there is an even more complex and probably not fully understood dynamics, which is beyond the picture of a simple reversible switching except for isolated fixed defect clusters.

Thus, the polaron-based luminescence is-like the luminescence from luminescent defect centers as markers-able to provide deeper insights into the physics of structural modifications in ferroelectrics.

On the one hand, the imperfect switching due to defect dipoles and the mechanism of their thermal realignment due to lithium ion mobility are now well understood. On the other hand, the details of the mechanism of luminescence domain contrast still lack an experimental elucidation. Up to now 
we do not really know if the radiative or the nonradiative recombination is directly affected by the frustrated defect complexes. Moreover, we do not know the mechanisms how these influences take place. Adopting the luminescence-quenching nonradiative recombination mechanism via bound hole polarons trapped at $\mathrm{Mg}_{\mathrm{Li}}+\mathrm{V}_{\mathrm{Li}}$ or also $\mathrm{Mg}_{\mathrm{V}}$-containing defect dipoles, the luminescence in the inverted domains may be reduced by increased attraction and trapping of electron or hole polarons by these frustrated dipoles. A saturation of this decay channel at higher excitation rates may even explain the contrast reduction at higher excitation intensities. However, only a deeper experimental investigation-of the nonradiative decay mechanisms themselves and of the influence of the frustrated defect dipoles—can clarify the real physical background of the luminescence domain contrast. This is also necessary to clear quantitatively the contribution of each species of defect dipoles $\left(\mathrm{Mg}_{\mathrm{Li}}+\mathrm{V}_{\mathrm{Li}}\right.$, $\mathrm{Mg}_{\mathrm{Nb}}+3 \mathrm{Mg}_{\mathrm{Li}}, \mathrm{Mg}_{\mathrm{V}}$ with lithium vacancies ...) to the domain contrast.

An interesting investigation to expand our knowledge of the domain contrasts would be annealing series to determine the domain contrasts and their saturation contrasts for a wide range of dopings and stoichiometries of LNO. The point would be to explore $C$ and $C^{\infty}$ as functions of the concentration of $\mathrm{Mg}_{\mathrm{Li}}+\mathrm{V}_{\mathrm{Li}}, \mathrm{Mg}_{\mathrm{V}}$ interstitial defects as well as of $\mathrm{Mg}_{\mathrm{Nb}}+3 \mathrm{Mg}_{\mathrm{Li}}$ clusters. Improving the excitation and detection efficiency would even allow to investigate the domain contrasts at low or zero dopings-as well as the contributions from the $\mathrm{Nb}_{\mathrm{Li}}+4 \mathrm{~V}_{\mathrm{Li}}, 5 \mathrm{Nb}_{\mathrm{Li}}+4 \mathrm{~V}_{\mathrm{Nb}}$ or $\mathrm{Nb}$-based defect complexes to these low-doping domain contrasts. On the technical side, the mentioned excitation intensity dependence of the contrast has always to be taken into account when carrying out future domain contrast measurements.

Due to limitations of our instrumentation we have only looked for domain contrasts of the visible polaronic luminescence. It may be worthwhile to test if the 1.3 and $1.5 \mathrm{eV}$ luminescence peaks show also domain contrasts, as well as the polaronic absorption bands. The mechanism of these luminescence peaks or of the decay of the polaronic absorption bands are not really comparable with the radiative polaronic recombination. If domain contrasts should be found in these cases, the mechanisms of such a domain contrast can expected to be quite different from the case of visible luminescence.

Author Contributions: P.R. and T.W. developed the idea and concept of this paper; P.R., A.T. and T.W. carried out the literature research and made an overview of the relevant information; P.R., T.W. and T.K. conceived the important experiments to be carried out; P.R. and T.K. performed the measurements and they evaluated, prepared and visualized the data. P.R., T.W., T.K., A.T., A.H., and L.M.E. discussed experiments to be made and the measurement results, resulting in the presented interpretation; P.R. validated several parts of the measurement results; R.S. provided the UV laser setup and supported the time-dependent measurements performed with this setup; Z.S., L.K., L.K., A.H., P.R. and T.W. provided the samples that have been used for the investigations; L.M.E., A.H. and T.W. supervised the work; L.M.E., L.K., A.H. and P.R. acquired the funding for the coauthors; P.R., T.W., A.H., T.K., L.M.E. and L.K. revised the paper; P.R. wrote the draft of the paper and put it into the final form.

Acknowledgments: P.R. thanks the Deutsche Forschungsgemeinschaft (DFG-German Science Foundation) for funding via the DFG priority program 1327, and the European Social Funds (ESF) through the ESF-NFG "Mind Nano". T.K. acknowledges the Cluster of Excellence "Center of Advancing Electronics Dresden" for funding. A.H. appreciates funding via DFG Research Grant No. HA 6982/1-1. We thank S. Neumann from the Leibniz-Institut für Festkörper-und Werkstoffforschung in Dresden for carrying out the high-temperature oxygen treatment of our lithium niobate samples. We also thank V. Gopalan for a helpful discussion.

Conflicts of Interest: The authors declare no conflict of interest.

\section{References}

1. Volk, T.; Wöhlecke, M. Lithium Niobate, Defects, Photorefraction and Ferroelectric Switching; Springer: Berlin/Heidelberg, Germany, 2008.

2. Schirmer, O.F.; Thiemann, O.; Wöhlecke, M. Defects in $\mathrm{LiNbO}_{3}$ I. Experimental aspects. J. Phys. Chem. Solids 1991, 52, 185-200, doi:10.1016/0022-3697(91)90064-7. [CrossRef]

3. Schirmer, O.F. $\mathrm{O}^{-}$bound small polarons in oxide materials. J. Phys. Condens. Mater. 2006, 18, R667-R704, doi:10.1088/0953-8984/18/43/R01. [CrossRef]

4. Schirmer, O.F.; Imlau, M.; Merschjann, C.; Schoke, B. Electron small polarons and bipolarons in $\mathrm{LiNbO}_{3}$. J. Phys. Condens. Mater. 2009, 21, 123201, doi:10.1088/0953-8984/21/12/123201. [CrossRef] [PubMed] 
5. Merschjann, C.; Schoke, B.; Conradi, D.; Imlau, M.; Corradi, G.; Polgár, K. Absorption cross sections and number densities of electron and hole polarons in congruently melting $\mathrm{LiNbO}_{3}$. J. Phys. Condens. Mater. 2009, 21, 015906, doi:10.1088/0953-8984/21/1/015906. [CrossRef] [PubMed]

6. Imlau, M.; Badorreck, H.; Merschjann, C. Optical nonlinearities of small polarons in lithium niobate. Appl. Phys. Rev. 2015, 2, 040606. [CrossRef]

7. Mhaouech, I.; Guilbert, L. Temperature dependence of small polaron population decays in iron-doped lithium niobate by Monte Carlo simulations. Solid State Sci. 2016, 60, 28-36. [CrossRef]

8. Von der Linde, D.; Schirmer, O.F.; Kurz, H. Intrinsic photorefractive effect of $\mathrm{LiNbO}_{3}$. Appl. Phys. A 1978, 15, 153-156, doi:10.1007/BF00928200. [CrossRef]

9. Schirmer, O.F.; von der Linde, D. Two-photon- and x-ray-induced $\mathrm{Nb}^{4+}$ and $\mathrm{O}^{-}$small polarons in $\mathrm{LiNbO}_{3}$. Appl. Phys. Lett. 1978, 33, 35-38, doi:10.1063/1.90181. [CrossRef]

10. Halliburton, L.E.; Sweeney, K.L.; Chen, C.Y. Electron Spin Resonance and Optical Studies of Point Defects in Lithium Niobate. Nucl. Instrum. Methods Phys. Res. B 1984, 1, 344-347. [CrossRef]

11. Arizmendi, L.; Cabrera, J.M.; Agulló-López, F. Defects induced in pure and doped $\mathrm{LiNbO}_{3}$ by irradiation and thermal reduction. J. Phys. C: Solid State Phys. 1984, 17, 515-529. [CrossRef]

12. Herth, P.; Schaniel, D.; Woike, Th.; Granzow, T.; Imlau, M.; Krätzig, E. Polarons generated by laser pulses in doped $\mathrm{LiNbO}_{3}$. Phys. Rev. B 2005, 71, 125128, doi:10.1103/PhysRevB.71.125128. [CrossRef]

13. Harhira, A.; Guilbert, L.; Bourson, P.; Rinnert, H. Decay time of polaron photoluminescence in congruent lithium niobate. Phys. Stat. Sol. C 2007, 4, 926-929, doi:10.1002/pssc.200673755. [CrossRef]

14. Harhira, A.; Guilbert, L.; Bourson, P.; Rinnert, H. Polaron luminescence in iron-doped lithium niobate. Appl. Phys. B 2008, 92, 555-561, doi:10.1007/s00340-008-3120-5. [CrossRef]

15. Klose, F.; Woehlecke, M.; Kapphan, S. Uv-excited luminescence of $\mathrm{LiNbO}_{3}$ and $\mathrm{LiNbO}_{3}: \mathrm{Mg}$. Ferroelectrics 1989, 92, 181-187, doi:10.1080/00150198908211324. [CrossRef]

16. Koppitz, J.; Schirmer, O.F.; Wöhlecke, M.; Kuznetsov, A.I.; Grabmaier, B.C. Threshold effects in LiNbO $2: \mathrm{Mg}$ caused by change of electron-lattice coupling. Ferroelectrics 1989, 92, 233-241, doi:10.1080/00150198908211331. [CrossRef]

17. Reichenbach, P.; Kämpfe, T.; Thiessen, A.; Schröder, M.; Haußmann, A.; Woike, T.; Eng, L.M. Multiphoton-induced luminescence contrast between antiparallel ferroelectric domains in Mg-doped $\mathrm{LiNbO}_{3}$. J. Appl. Phys. 2014, 115, 213509, doi:10.1063/1.4881496. [CrossRef]

18. Reichenbach, P.; Kämpfe, T.; Thiessen, A.; Haußmann, A.; Woike, T.; Eng, L.M. Multiphoton photoluminescence contrast in switched $\mathrm{Mg}: \mathrm{LiNbO}_{3}$ and $\mathrm{Mg}: \mathrm{LiTaO}_{3}$ single crystals. Appl. Phys. Lett. 2014, 105, 122906, doi:10.1063/1.4896579. [CrossRef]

19. Kämpfe, T.; Haußmann, A.; Eng, L.M.; Reichenbach, P.; Thiessen, A.; Woike, T.; Steudtner, R. Time-resolved photoluminescence spectroscopy of $\mathrm{Nb}^{4+}$ and $\mathrm{O}^{-}$polarons in $\mathrm{LiNbO}_{3}$ single crystals. Phys. Rev. B 2016, 93, 174116, doi:10.1103/PhysRevB.93.174116. [CrossRef]

20. Scott, J.G.; Mailis, S.; Sones, C.L.; Eason, R.W. A Raman study of single-crystal congruent lithium niobate following electric-field repoling. Appl. Phys. A 2004, 79, 691-696. [CrossRef]

21. Stone, G.; Knorr, B.; Gopalan, V.; Dierolf, V. Frequency shift of Raman modes due to an applied electric field and domain inversion in LiNbO. Phys. Rev. B 2011, 84, 134303. [CrossRef]

22. Nataf, G.F.; Guennou, M.; Haußmann, A.; Barrett, N.; Kreisel, J. Evolution of defect signatures at ferroelectric domain walls in $\mathrm{Mg}$-doped $\mathrm{LiNbO}_{3}$. Phys. Stat. Sol. RRL 2015, 10, 222-226. [CrossRef]

23. Dierolf, V.; Sandmann, C. Inspection of periodically poled waveguide devices by confocal luminescence microscopy. Appl. Phys. B 2004, 78, 363-366, doi:10.1007/s00340-003-1377-2. [CrossRef]

24. Gopalan, V.; Gupta, M.C. Observation of internal field in $\mathrm{LiTaO}_{3}$ single crystals: Its origin and time-temperature dependence. Appl. Phys. Lett. 1996, 68, 888-890, doi:10.1063/1.116220. [CrossRef]

25. Gopalan, V.; Gupta, M.C. Origin of internal field and visualization of $180^{\circ}$ domains in congruent $\mathrm{LiTaO}_{3}$ crystals. J. Appl. Phys. 1996, 80, 6099-6106. [CrossRef]

26. Gopalan, V.; Gupta, M.C. Origin and characteristics of internal fields in $\mathrm{LiNbO}_{3}$ crystals. Ferroelectrics 1997, 198, 49-59, doi:10.1080/00150199708228337. [CrossRef]

27. Gopalan, V.; Mitchell, T.E.; Furukawa, Y.; Kitamura, K. The role of nonstoichiometry in $180^{\circ}$ domain switching of $\mathrm{LiNbO}_{3}$ crystals. Appl. Phys. Lett. 1998, 72, 1981-1983. [CrossRef]

28. Gopalan, V.; Dierolf, V.; Scrymgeour, D.A. Defect-domain wall interactions in trigonal ferroelectrics. Annu. Rev. Mater. Res. 2007, 37, 449-489, doi:10.1146/annurev.matsci.37.052506.084247. [CrossRef] 
29. Kim, S.; Gopalan, V.; Kitamura, K.; Furukawa, Y. Domain reversal and nonstoichiometry in lithium tantalate. J. Appl. Phys. 2001, 90, 2949-2963, doi:10.1063/1.1389525. [CrossRef]

30. Li, Y.; Schmidt, W.G.; Sanna, S. Defect complexes in congruent $\mathrm{LiNbO}_{3}$ and their optical signatures. Phys. Rev. B 2015, 91, 174106. [CrossRef]

31. Yatsenko, A.V.; Ivanova, E.N.; Sergeev, N.A. NMR study of intrinsic defects in congruent $\mathrm{LiNbO}_{3}$. 1. “Unoverlapping defects". Physica B 1997, 240, 254-262. [CrossRef]

32. Yatsenko, A.V.; Ivanova-Maksimova, H.M.; Sergeev, N.A. NMR study of intrinsic defects in congruent $\mathrm{LiNbO}_{3}$. 2. “Overlapping defects". Physica B 1998, 254, 256-259. [CrossRef]

33. Zotov, N.; Boysen, H.; Frey, F.; Metzger, T.; Born, E. Cation substitution models of congruent $\mathrm{LiNbO}_{3}$ investigated by X-ray and neutron powder diffraction. J. Phys. Chem. Solids 1994, 55, 145-152. [CrossRef]

34. Friedrich, M.; Schmidt, W.G.; Schindlmayr, A.; Sanna, S. Polaron optical absorption in congruent lithium niobate from time-dependent density-functional theory. Phys. Rev. Mater. 2017, 1, 054406. [CrossRef]

35. Kling, A.; Kollewe, D.; Grabmaier, B.C. Lattice site investigations for $\mathrm{Mg}$ in $\mathrm{LiNbO}_{3}$ by combined RBS-PIXE-NRA channeling experiments. Nucl. Instrum. Methods Phys. Res. B 1992, 64, 232-236. [CrossRef]

36. Furukawa, Y.; Kitamura, K.; Takekawa, S.; Niwa, K.; Yajima, Y.; Iyi, N.; Mnushkina, I.; Guggenheim, P.; Martin, J.M. The correlation of $\mathrm{MgO}$-doped near-stoichiometric $\mathrm{LiNbO}_{3}$ composition to the defect structure. J. Cryst. Growth 2000, 211, 230-236. [CrossRef]

37. Péter, Á.; Polgár, K.; Kovács, L.; Lengyel, K. Threshold concentration of MgO in near-stoichiometric $\mathrm{LiNbO}_{3}$ crystals. J. Cryst. Growth 2005, 284, 149-155. [CrossRef]

38. Volk, T.; Rubinina, N.; Wöhlecke, M. Optical-damage-resistant impurities in lithium niobate. J. Opt. Soc. Am. B 1994, 11, 1681-1687. [CrossRef]

39. Redfield, D.; Burke, W.J. Optical absorption edge of $\mathrm{LiNbO}_{3}$. J. Appl. Phys. 1974, 45, 4566-4571. [CrossRef]

40. Kase, S.; Ohi, K. Optical absorption and interband faraday rotation in $\mathrm{LiTaO}_{3}$ and $\mathrm{LiNbO}_{3}$. Ferroelectrics 1974, 8, 419-420. [CrossRef]

41. Bhatt, R.; Bhaumik, I.; Ganesamoorthy, S.; Karnal, A.K.; Swami, M.K.; Patel, H.S.; Gupta, P.K. Urbach tail and bandgap analysis in near stoichiometric $\mathrm{LiNbO}_{3}$ crystals. Phys. Stat. Sol. A 2012, 209, 176-180. [CrossRef]

42. Xin, F.; Zhai, Z.; Wang, X.; Kong, Y.; Xu, J.; Zhang, G. Threshold behavior of the Einstein oscillator, electron-phonon interaction, band-edge absorption, and small hole polarons in $\mathrm{LiNbO}_{3}: \mathrm{Mg}$ crystals. Phys. Rev. B 2012, 86, 165132. [CrossRef]

43. Kovács, L.; Ruschhaupt, G.; Polgár, K.; Corradi, G.; Wöhlecke, M. Composition dependence of the ultraviolet absorption edge in lithium niobate. Appl. Phys. Lett. 1997, 70, 2801-2803, doi:10.1063/1.119056 [CrossRef]

44. Wiesendanger, E.; Güntherodt, G. Optical anisotropy of $\mathrm{LiNbO}_{3}$ and $\mathrm{KNbO}_{3}$ in the interband transition region. Solid State Commun. 1974, 14, 303-306. [CrossRef]

45. Mamedov, A.M.; Osman, M.A.; Hajieva, L.C. VUV Reflectivity of $\mathrm{LiNbO}_{3}$ and $\mathrm{LiTaO}_{3}$ Single Crystals. Appl. Phys. A 1984, 34, 189-192. [CrossRef]

46. Mamedov, A.M.; Hajiyeva, L.S.; Ibragimova, I.S.; Aliyeva, B.S. Vacuum ultraviolet reflectivity (VUV) and electron states in $\mathrm{LiNbO}_{3}$. Physica B 1985, 128, 61-68. [CrossRef]

47. Thierfelder, C.; Sanna, S.; Schindlmayr, A.; Schmidt, W.G. Do we know the band gap of lithium niobate? Phys. Stat. Sol. C 2010, 7, 362-365. [CrossRef]

48. Riefer, A.; Friedrich, M.; Sanna, S.; Gerstmann, U.; Schindlmayr, A.; Schmidt, W.G. LiNbO 3 electronic structure: Many-body interactions, spin-orbit coupling, and thermal effects. Phys. Rev. B 2016, 93, 075205. [CrossRef]

49. Riefer, A.; Sanna, S.; Schindlmayr, A.; Schmidt, W.G. Optical response of stoichiometric and congruent lithium niobate from first-principles calculations. Phys. Rev. B 2013, 87, 195208. [CrossRef]

50. Beyer, O.; Maxein, D.; Buse, K.; Sturman, B.; Hsieh, H.T.; Psaltis, D. Investigation of nonlinear absorption processes with femtosecond light pulses in lithium niobate crystals. Phys. Rev. E 2005, 71, 056603. [CrossRef] [PubMed]

51. Beyer, O.; Maxein, D.; Buse, K.; Sturman, B.; Hsieh, H.T.; Psaltis, D. Femtosecond time-resolved absorption processes in lithium niobate crystals. Opt. Lett. 2005, 30, 1366-1368. [CrossRef] [PubMed]

52. Qiu, Y.; Ucer, K.B.; Williams, R.T. Formation time of a small electron polaron in $\mathrm{LiNbO}_{3}$ : Measurements and interpretation. Phys. Stat. Sol. C 2005, 2, 232-235. [CrossRef] 
53. Koppitz, J.; Schirmer, O.F.; Kuznetsov, A.I. Thermal Dissociation of Bipolarons in Reduced Undoped $\mathrm{LiNbO}_{3}$. Europhys. Lett. 1987, 4, 1055-1059. [CrossRef]

54. Jermann, F.; Simon, M.; Bower, R.; Krätzig, E.; Schirmer, O.F. Light-induced absorption changes in reduced lithium niobate. Ferroelectrics 1995, 165, 319-327. [CrossRef]

55. Merschjann, C.; Berben, D.; Imlau, M.; Wöhlecke, M. Evidence for Two-Path Recombination of Photoinduced Small Polarons in Reduced $\mathrm{LiNbO}_{3}$. Phys. Rev. Lett. 2006, 96, 186404. [CrossRef] [PubMed]

56. Merschjann, C.; Schoke, B.; Imlau, M. Influence of chemical reduction on the particular number densities of light-induced small electron and hole polarons in nominally pure $\mathrm{LiNbO}_{3}$. Phys. Rev. B 2007, 76, 085114 . [CrossRef]

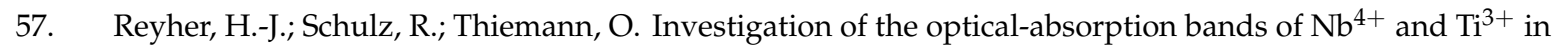
lithium niobate using magnetic circular dichroism and optically detected magnetic-resonance techniques. Phys. Rev. B 1994, 50, 3609. [CrossRef]

58. Faust, B.; Müller, H.; Schirmer, O.F. Free small polarons in $\mathrm{LiNbO}_{3}$. Ferroelectrics 1994, 153, $297-302$. [CrossRef]

59. Kurz, H.; Krätzig, E.; Keune, W.; Engelmann, H.; Gonser, U.; Dischler, B.; Räuber, A. Photorefractive Centers in $\mathrm{LiNbO}_{3}$, Studied by Optical-, Mössbauer- and EPR-Methods. Appl. Phys. A 1977, 12, 355-368. [CrossRef]

60. Reik, H.G.; Heese, D. Frequency dependence of the electrical conductivity of small polarons for high and low temperatures. J. Phys. Chem. Solids 1967, 28, 581-596. [CrossRef]

61. Emin, D. Optical properties of large and small polarons and bipolarons. Phys. Rev. B 1993, 48, 13691. [CrossRef]

62. Eliseev, E.A.; Morozovska, A.N.; Svechnikov, G.S.; Gopalan, V.; Shur, V.Ya. Static conductivity of charged domain wall in uniaxial ferroelectric-semiconductors. Phys. Rev. B 2011, 83, 235313. [CrossRef]

63. Bogomolov, V.N.; Kudinov, E.K.; Mirlin, D.N.; Firsov, Yu.A. Polaron Mechanism of Light Absorption in Rutile Crystals $\left(\mathrm{TiO}_{2}\right)$. Sov. Phys. Solid State 1968, 9, 1630-1639.

64. Wang, Q.; Leng, S.; Yu, Y. Activation Energy of Small Polarons and Conductivity in $\mathrm{LiNbO}_{3}$ and $\mathrm{LiTaO}_{3}$ Crystals. Phys. State Solid B 1996, 194, 661-665. [CrossRef]

65. Schröder, M.; Haußmann, A.; Thiessen, A.; Soergel, E.; Woike, T.; Eng, L.M. Conducting Domain Walls in Lithium Niobate Single Crystals. Adv. Funct. Mater. 2012, 22, 3936-3944. [CrossRef]

66. Austin, I.G.; Mott, N.F. Polarons in crystalline and non-crystalline materials. Adv. Phys. 1969, 18, 41-102. [CrossRef]

67. Holstein, T. Studies of Polaron Motion, Part I. Ann. Phys. (N.Y.) 1959, 8, 325-342. [CrossRef]

68. Holstein, T. Studies of Polaron Motion, Part II. Ann. Phys. (N.Y.) 1959, 8, 343-389. [CrossRef]

69. Rubinstein, M. Two-component model of polaronic transport. J. Appl. Phys. 2000, 87, 5019-5021. [CrossRef]

70. Schirmer, O.F. Holes bound as small polarons to acceptor defects in oxide materials: Why are their thermal ionization energies so high? J. Phys. Condens. Matter 2011, 23, 334218. [CrossRef] [PubMed]

71. Herth, P.; Granzow, T.; Schaniel, D.; Woike, Th.; Imlau, M.; Krätzig, E. Evidence for Light-Induced Hole Polarons in $\mathrm{LiNbO}_{3}$. Phys. Rev. Lett. 2005, 95, 067404. [CrossRef] [PubMed]

72. Donnerberg, H.; Tomlinson, S.M.; Catlow, C.R.A.; Schirmer, O.F. Computer-simulation studies of extrinsic defects in $\mathrm{LiNbO}_{3}$ crystals. Phys. Rev. B 1991, 44, 4877. [CrossRef]

73. Rebouta, L.; da Silva, M.F.; Soares, J.C.; Hage-Ali, M.; Stoquert, J.P.; Siffert, P.; Sanz-García, J.A.; Diéguez, E.; Agulló-López, F. Lattice Site of Iron in $\mathrm{LiNbO}_{3}\left(\mathrm{Fe}^{3+}\right)$ by the PIXE/Channelling Technique. Europhys. Lett. 1991, 14, 557-561. [CrossRef]

74. Clark, M.G.; DiSalvo, F.J.; Glass, A.M.; Peterson, G.E. Electronic structure and optical index damage of iron-doped lithium niobate. J. Chem. Phys. 1973, 59, 6209-6219. [CrossRef]

75. Berben, D.; Buse, K.; Wevering, S.; Herth, P.; Imlau, M.; Woike, T. Lifetime of small polarons in iron-doped lithium-niobate crystals. J. Appl. Phys. 2000, 87, 1034-1041, doi:10.1063/1.371976 [CrossRef]

76. Merschjann, C.; Imlau, M.; Brüning, H.; Schoke, B.; Torbrügge, S. Nonexponential relaxation dynamics of localized carrier densities in oxide crystals without structural or energetic disorder. Phys. Rev. B 2011, 84, 52302. [CrossRef]

77. Thomas, D.G.; Hopfield, J.J.; Augustyniak, W.M. Kinetics of Radiative Recombination at Randomly Distributed Donors and Acceptors. Phys. Rev. A 1965, 140, 202-220. [CrossRef] 
78. Zatryb, G.; Podhorodecki, A.; Misiewicz, J.; Cardin, J.; Gourbilleau, F. On the nature of the stretched exponential photoluminescence decay for silicon nanocrystals. Nanoscale Res. Lett. 2011, 6, 106. [CrossRef] [PubMed]

79. Parsons, R.; Cornish, W.D.; Young, L. Luminescence due to iron centers in lithium niobate. Appl. Phys. Lett. 1975, 27, 654-656. [CrossRef]

80. Phillips, W.; Staebler, D.L. Control of the $\mathrm{Fe}^{2+}$ concentration in iron-doped lithium niobate. J. Electron. Mater. 1974, 3, 601-617. [CrossRef]

81. Staebler, D.L.; Phillips, W. Fe-Doped $\mathrm{LiNbO}_{3}$ for Read-Write Applications. Appl. Opt. 1974, 13, 788-794. [CrossRef] [PubMed]

82. Kostritskii, S.M.; Aillerie, M.; Margueron, S.; Bourson, P. Two-photon luminescence of small polarons in reduced $\mathrm{LiNbO}_{3}$ crystals. IOP Conf. Ser. Mater. Sci. Eng. 2010, 15, 012057, doi:10.1088/1757-899X/15/1/012057. [CrossRef]

83. Harhira, A. Photoluminescence Polaron dans le Niobate de Lithium: Approche Expérimentale et Modélisation. Ph.D. Thesis, Université Paul Verlaine, Metz, France, 2007.

84. Kostritskii, S.M.; Aillerie, M.; Margueron, S.; Bourson, P. Gated luminescence in as-grown and reduced undoped $\mathrm{LiNbO}_{3}$ crystals. J. Phys. Conf. Ser. 2013, 416, 012033. [CrossRef]

85. Krol, D.M.; Blasse, G.; Powell, R.C. The influence of the $\mathrm{Li} / \mathrm{Nb}$ ratio on the luminescence properties of $\mathrm{LiNbO}_{3}$. J. Chem. Phys. 1980, 73, 163-166. [CrossRef]

86. Tang, L.-Q.; Zhao, L.-J.; Zhang, X.-Z.; Yu, H.; Meng, J.; Liang, Q.; Xu, J.-J.; Kong, Y.-F. Luminescent enhancement in Mg-and Er-codoped $\mathrm{LiNbO}_{3}$ crystals. Chin. Phys. Lett. 2005, 22, 588-590.

87. Ryba-Romanowski, W.; Gołab, S.; Dominiak-Dzik, G.; Palatnikov, M.N.; Sidorov, N.V. Influence of temperature on luminescence of terbium ions in $\mathrm{LiNbO}_{3}$. Appl. Phys. Lett. 2001, 78, 3610-3611. [CrossRef]

88. Galutskiy, V.V.; Ignatyev, B.V.; Isaev, V.A.; Lebedev, V.A.; Avanesov, A.G.; Mihaylenko, A.L.; Stroganova, E.V.; Brik, M.G. $\mathrm{Cr}^{3+}$ luminescence quenching in stoichiometric lithium niobate crystals. J. Non-Cryst. Solids 2006, 352, 2395-2398. [CrossRef]

89. Kovács, L.; Szaller, Z.; Lengyel, K.; Corradi, G. Hydroxyl ions in stoichiometric $\mathrm{LiNbO}_{3}$ crystals doped with optical damage resistant ions. Opt. Mater. 2014, 37, 55-58. [CrossRef]

90. Lengyel, K.; Péter, A.; Kovács, L.; Corradi, G.; Pálfalvi, L.; Hebling, J.; Unferdorben, M.; Dravecz, G.; Hajdara, I.; Szaller, Z.; et al. Growth, defect structure and THz application of stoichiometric lithium niobate. Appl. Phys. Rev. 2015, 2, 040601. [CrossRef]

91. Kovács, L.; Szaller, Z.; Lengyel, K.; Péter, A.; Hajdara, I.; Mandula, G.; Pálfalvi, L.; Hebling, J. Photorefractive damage resistance threshold in stoichiometric $\mathrm{LiNbO}_{3}$ :Zr crystals. Opt. Lett. 2013, 38, 2861-2864. [CrossRef] [PubMed]

92. Kong, Y.; Liu, S.; Zhao, Y.; Liu, H.; Chen, S.; Xu, J. Highly optical damage resistant crystal: Zirconium-oxide-doped lithium niobate. Appl. Phys. Lett. 2007, 91, 081908. [CrossRef]

93. Bridges, F.; Mackeen, C.; Kovács, L. No difference in local structure about a Zn dopant for congruent and stoichiometric $\mathrm{LiNbO}_{3}$. Phys. Rev. B 2016, 94, 014101. [CrossRef]

94. Dierolf, V.; Sandmann, C.; Kim, S.; Gopalan, V.; Polgar, K. Ferroelectric domain imaging by defect-luminescence microscopy. J. Appl. Phys. 2003, 93, 2295-2297, doi:10.1063/1.1538333. [CrossRef]

95. Dierolf, V.; Sandmann, C. Combined excitation emission spectroscopy of defects for site-selective probing of ferroelectric domain inversion in lithium niobate. J. Lumin. 2007, 125, 67-79, doi:10.1016/j.jlumin.2006.08.054. [CrossRef]

96. Jia, Y.; Wei, Y.; Wu, Z.; Zhou, Z.; Chen, J.; Jia, Y.; Zhang, Y.; Zhou, J.; Wang, H. Effects of compositional changes on luminescence of lead-free $\mathrm{Eu}^{3+}$-doped $\mathrm{K}_{1-\mathrm{x}} \mathrm{Na}_{\mathrm{x}} \mathrm{NbO}_{3}$ piezoelectric ceramics. J. Alloys Compd. 2014, 586, 66-68. [CrossRef]

97. Tian, X.; Wu, Z.; Jia, Y.; Chen, J.; Zheng, R.K.; Zhang, Y.; Luo, H. Remanent-polarization-induced enhancement of photoluminescence in $\mathrm{Pr}^{3+}$-doped lead-free ferroelectric $\left(\mathrm{Bi}_{0.5} \mathrm{Na}_{0.5}\right) \mathrm{TiO}_{3}$ ceramic. Appl. Phys. Lett. 2013, 102, 042907. [CrossRef]

98. Soergel, E. Visualization of ferroelectric domains in bulk single crystals. Appl. Phys. B 2005, 81, 729-751. [CrossRef]

99. Wulf, K.; Müller, H.; Schirmer, O.F.; Grabmaier, B.C. AC response of reduced undoped and Mg-doped $\mathrm{LiNbO}_{3}$. Radiat. Eff. Def. Solids 1991, 119-121, 687-692. [CrossRef] 
100. Que, W.; Lim, S.; Zhang, L.; Yao, X. The Magnesium Diffused Layer Characteristics of a Lithium Niobate Single Crystal with Magnesium-Ion Indiffusion. Jpn. J. Appl. Phys. 1998, 37, 903. [CrossRef]

101. Bollmann, W.; Stöhr, H.-J. Incorporation and mobility of $\mathrm{OH}^{-}$ions in $\mathrm{LiNbO}_{3}$ crystals. Phys. Stat. Sol. A 1977, 39, 477-484. [CrossRef]

102. Buse, K.; Breer, S.; Peithmann, K.; Kapphan, S.; Gao, M.; Krätzig, E. Origin of thermal fixing in photorefractive lithium niobate crystals. Phys. Rev. B 1997, 56, 1225. [CrossRef]

103. Bollmann, W.; Gernand, M. On the Disorder of $\mathrm{LiNbO}_{3}$ Crystals. Phys. State Solid A 1972, 9, $301-308$. [CrossRef]

104. Birnie, D.P., III. Analysis of diffusion in lithium niobate. J. Mater. Sci. 1993, 28, 302-315. [CrossRef]

105. Ohlendorf, G.; Richter, D.; Sauerwald, J.; Fritze, H. High-Temperature Electrical Conductivity and Electromechanical Properties of Stoichiometric Lithium Niobate. Diffus. Fundam. 2008, 8, 6.

106. Heitjans, P.; Masoud, M.; Feldhoff, A.; Wilkening, M. NMR and impedance studies of nanocrystalline and amorphous ion conductors: lithium niobate as a model system. Faraday Discuss. 2007, 134, 67-82. [CrossRef] [PubMed]

107. Bordui, P.F.; Norwood, R.G.; Bird, C.D.; Carella, J.T. Stoichiometry issues in single-crystal lithium tantalate. J. Appl. Phys. 1995, 78, 4647-4650. [CrossRef]

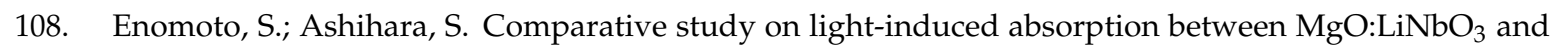
MgO:LiTaO 3 . J. Appl. Phys. 2011, 110, 063111. [CrossRef]

109. Sheng, Y.; Best, A.; Butt, H.-J.; Krolikowski, W.; Arie, A.; Koynov, K. Three-dimensional ferroelectric domain visualization by Čerenkov-type second harmonic generation. Opt. Express 2010, 18, 16539-16545. [CrossRef] [PubMed]

110. Kämpfe, T.; Reichenbach, P.; Schröder, M.; Haußmann, A.; Eng, L.M.; Woike, T.; Soergel, E. Optical three-dimensional profiling of charged domain walls in ferroelectrics by Cherenkov second-harmonic generation. Phys. Rev. B 2014, 89, 035314. [CrossRef]

111. Kämpfe, T.; Reichenbach, P.; Haußmann, A.; Woike, T.; Soergel, E.; Eng, L.M. Real-time three-dimensional profiling of ferroelectric domain walls. Appl. Phys. Lett. 2015, 107, 152905. [CrossRef]

112. Yang, T.J.; Gopalan, V.; Swart, P.J.; Mohideen, U. Direct Observation of Pinning and Bowing of a Single Ferroelectric Domain Wall. Phys. Rev. Lett. 1999, 82, 4106. [CrossRef]

(C) 2018 by the authors. Licensee MDPI, Basel, Switzerland. This article is an open access article distributed under the terms and conditions of the Creative Commons Attribution (CC BY) license (http://creativecommons.org/licenses/by/4.0/). 